\title{
L-Piperazine-2-carboxylic Acid Derived $N$-Formamide as Highly Enantioselective Lewis Basic Catalyst for Hydrosilylation of $N$-Aryl Imines with Unprecedented Substrate Profile
}

Zhouyu Wang, ${ }^{\dagger} \S$ Mounuo Cheng, ${ }^{\dagger}$ Pengcheng Wu, ${ }^{\dagger, \dagger}$ Siyu Wei, ${ }^{\dagger}$ Jian Sun ${ }^{\dagger *}$

${ }^{\dagger}$ Chengdu Institute of Biology, Chinese Academy of Sciences, Chengdu, 610041 (China)

Fax: (+86) 28-85222753

${ }^{\ddagger}$ Chengdu Institute of Organic Chemistry, Chinese Academy of Sciences, Chengdu, 610041 (China)

${ }^{\S}$ Xihua University, Chengdu, 610039 (China)

sunjian@cib.ac.cn

\section{(Supporting Information)}

General Information: All starting materials were of the highest commercially available grade and used without further purification. All solvents used in the reactions were distilled from appropriate drying agents prior to use. Reactions were monitored by thin layer chromatography using silica gel HSGF254 plates. Flash chromatography was performed using silica gel HG/T2354-92. ${ }^{1} \mathrm{H}$ - and ${ }^{13} \mathrm{C}$ NMR (300 or 600 and 75 or $150 \mathrm{~Hz}$, respectively) spectra were recorded in $\mathrm{CDCl}_{3}$ or DMSO. ${ }^{1} \mathrm{H}$ NMR chemical shifts are reported in ppm $(\boldsymbol{\delta})$ relative to tetramethylsilane (TMS) with the solvent resonance employed as the internal standard $\left(\mathrm{CDCl}_{3}, \boldsymbol{\delta} 7.26 \mathrm{ppm}\right.$; DMSO, $\left.\boldsymbol{\delta} 2.36 \mathrm{ppm}\right)$. Data are reported as follows: chemical shift, multiplicity $(\mathrm{s}=$ singlet, $\mathrm{d}=$ doublet, $\mathrm{q}=$ quartet, $\mathrm{m}=$ multiplet $)$, coupling constants $(\mathrm{Hz})$ and integration. ${ }^{13} \mathrm{C}$ NMR chemical shifts are reported in ppm from tetramethylsilane (TMS) with the solvent resonance as the internal standard $\left(\mathrm{CDCl}_{3}, \boldsymbol{\delta} 77.0 \mathrm{ppm}\right.$; DMSO, $\boldsymbol{\delta} 40.0$ ppm). ESIMS spectra were recorded on BioTOF Q. HPLC analyses were performed on PerkinElmer (Series 200 UV/VIS Detector and Series 200 Pump). Chiralpak OD-H, AD-H and $\mathrm{OJ}-\mathrm{H}$ columns were purchased from Daicel Chemical Industries, LTD. All enantiomer ratios have been controlled by co-injections of the pure sample with the racemic substrates. All imines were prepared according to the general procedure. ${ }^{1}$ Imines $\mathbf{6 a - g}, \mathbf{6 i}, \mathbf{6 0}$ and $\mathbf{6 p}$

\footnotetext{
${ }^{1}$ (a) Samec, J. S. M.; Bäckvall, J. E. Chem. Eur. J. 2002, 8, 2955. (b) Malkov, A. V.; Mariani, A.; MacDougall, K. N.; Kocovsky, P. Org. Lett. 2004, 6, 2253. (c) Gawinecki, R. Pol. J. Chem. 1987, 61, 589. (d) Barluenga, A.; Aznar, F. Synthesis 1976, 704. (e) Hansen, M. C.; Buchwald, S. L. Org. Lett. 2000, 2, 713. (f) Xiao, D. M.; Zhang, X. M. Angew. Chem..Int. Ed. 2001, 40, 3425. (g) Barluenga, J.; Fernàndez, M. A.; Aznar, F.; Valdès, C. Chem. Eur. J. 2004, 10, 494. (h) Periasamy, M.; Srinivas, G.; Bharath, P. J. org. chem., 1999, 64, 4204. (i) Ozawa, F.; Okamoto, H.; Kawagishi, S.; Yamamoto, S.; Minami, T.; Yoshifuji, M. J. Am. Chem. Soc. 2002, 124, 10968. (j)
} 
and amines 7a-g, 7i, 7o and 7p are known compounds. ${ }^{1}$ Chemical yields refer to pure isolated substances.

\section{Scheme for the synthesis of 4a-e:}

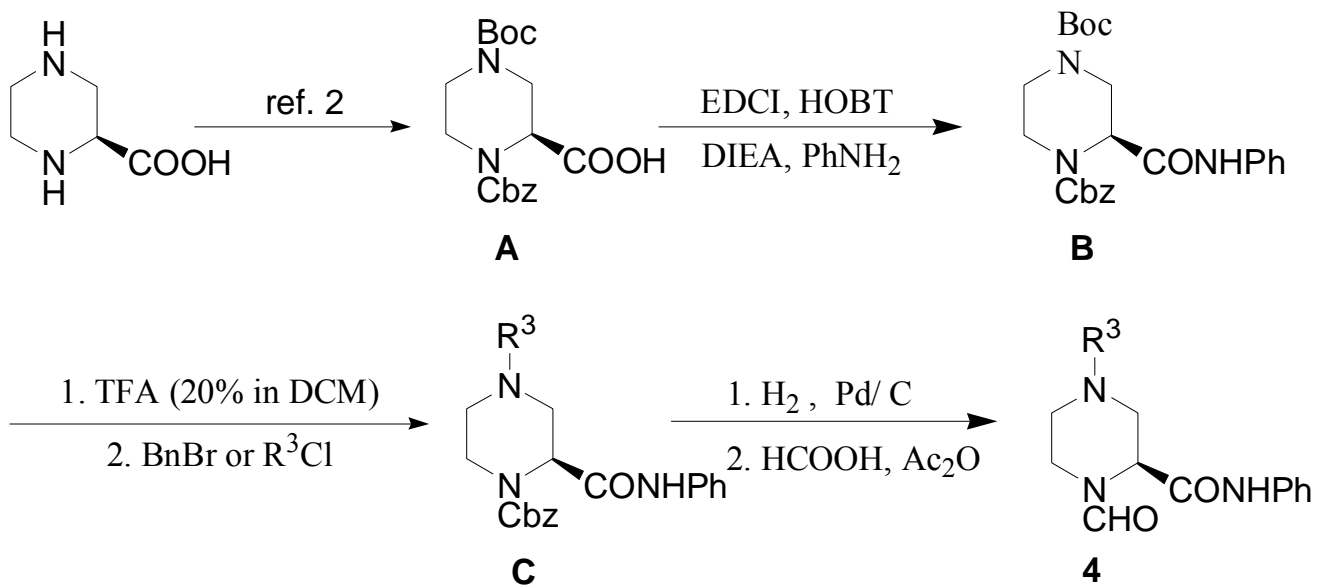

$$
\begin{aligned}
& \text { a, } \mathrm{R}^{3}=\mathrm{Bn} ; \mathbf{b}, \mathrm{R}^{3}=\mathrm{SO}_{2} \mathrm{Me} ; \mathbf{c}, \mathrm{R}^{3}=\mathrm{SO}_{2} \mathrm{Ph} \\
& \text { d, } \mathrm{R}^{3}=\mathrm{SO}_{2}(p-\mathrm{MePh}) ; \mathbf{e}, \mathrm{R}^{3}=\mathrm{SO}_{2}\left(p-{ }_{\mathrm{B}} \mathrm{BuPh}\right)
\end{aligned}
$$

\section{Procedure for the synthesis of $B$ :}

To a solution of $\mathbf{A}(10 \mathrm{~g}, 27.4 \mathrm{mmol})$ in DCM (70 mL) was added aniline (3 mL, $32.9 \mathrm{mmol})$, DIEA $(5.8 \mathrm{~mL}, 32.9 \mathrm{mmol}), \mathrm{HOBt}(4.8 \mathrm{~g}, 32.9 \mathrm{mmol})$ and EDCI $(6.3 \mathrm{~g}, 32.9 \mathrm{mmol})$ at $0{ }^{\circ} \mathrm{C}$. The reaction mixture was stirred at room temperature for $12 \mathrm{~h}$, and then concentrated under reduced pressure. The residue was diluted with EtOAc $(200 \mathrm{~mL})$ and the solution was washed with saturated aqueous $\mathrm{NaHCO}_{3}(40 \mathrm{~mL})$, aqueous $\mathrm{HCl}(1.0 \mathrm{~N}, 20 \mathrm{~mL})$ and brine $(20 \mathrm{~mL})$ and dried over anhydrous $\mathrm{Na}_{2} \mathrm{SO}_{4}$. After removal of solvents under reduced pressure, the residue was purified through column chromatography on silica gel (eluent: hexane/EtOAc $=$ 4/1) to give pure $\mathbf{B}$.

\section{Procedure for the synthesis of $\mathrm{C}$ :}

Compound B (1.0 g, $2.28 \mathrm{mmol})$ was dissolved in $20 \mathrm{v} \%$ TFA in $\mathrm{CH}_{2} \mathrm{Cl}_{2}(40 \mathrm{~mL})$ for an hour and then concentrated under reduced pressure. The residue was dissolved in DCM and the

Wang, Z.; Ye, X.; Wei, S.; Wu, P.; Zhang, A.; Sun, J. Org. Lett. 2006, 8, 999. (k) Anna, T.; Jarle, S. D.; Pher, G. A. Chem. Eur. J. 2006, 12, 2318.

${ }^{2}$ Dorsey, B. D.; Levin, R. B.; McDaniel, S. L.; Vacca, J. P.; Guare, J. P.; Darke, P. L.; Zugay, J. A.; Emini, E. A.; Schleif, W. A.; Quintero, J. C.; Lin, J. H.; Chen, I. W.; Holloway, M. K.; Fitzgerald, P. M.; Axel, M. G.; Osovic, D.; Anderson, P. S.; Huff, J. R. J. Med. Chem. 1994, 37, 3443. 
resulting solution was cooled to $0{ }^{\circ} \mathrm{C}$. TEA $(480 \mu \mathrm{L}, 3.42 \mathrm{mmol})$ was added, followed by the addition of $\mathrm{BnBr}$ (for $\mathbf{4 a}$ ) or $\mathrm{R}^{3} \mathrm{Cl}$ (for $\mathbf{4 b}$-e). The mixture was allowed to stir at room temperature overnight. After removal of solvents under reduced pressure, the residue was purified though column chromatography on silica gel to afford pure $\mathbf{C}$.

\section{Procedure for the synthesis of 4:}

Compound C (500 mg), 5\% Pd/C (50 mg) and methanol (15 mL) were charged in a two-neck flask $(100 \mathrm{~mL})$. The mixture was stirred under hydrogen $(1 \mathrm{~atm})$ until $\mathbf{C}$ disappeared completely (monitored by TLC), and then filtered through celite. The filtrate was concentrated under reduced pressure. The residue was dissolved in formic acid (1.5 mL) and the resulting solution was cooled to $0{ }^{\circ} \mathrm{C}$. Acetic anhydride $(1 \mathrm{~mL})$ was added dropwise and the mixture was allowed to stir at room temperature overnight. After removal of solvents under reduced pressure, the residue was purified though column chromatography on silica gel to give 4 .

4a: white solid; yield: $80 \% ;[\alpha]_{\mathrm{D}}{ }^{20}=-83.0\left(\mathrm{c}=0.10, \mathrm{CHCl}_{3}\right) ; \operatorname{mp} 54.0-55.0{ }^{\circ} \mathrm{C} ;{ }^{1} \mathrm{H}$ NMR $\left(600 \mathrm{MHz}, \mathrm{CDCl}_{3}\right): \boldsymbol{\delta}=2.15-2.20(\mathrm{~m}, 1 \mathrm{H}), 2.23 \& 2.42(\mathrm{dd}, J=12.18,3.96 \mathrm{~Hz}, 1 \mathrm{H}), 3.03-$ $3.09(\mathrm{~m}, 1 \mathrm{H}), 3.38 \& 3.46(\mathrm{~d}, J=12.18 \mathrm{~Hz}, 1 \mathrm{H}), 3.55-3.68(\mathrm{~m}, 3 \mathrm{H}), 4.13 \& 5.09(\mathrm{~s}, 1 \mathrm{H}), 4.34$ $(\mathrm{dd}, J=12.60,2.70 \mathrm{~Hz}, 1 \mathrm{H}), 7.11-7.15(\mathrm{~m}, 1 \mathrm{H}), 7.28-7.40(\mathrm{~m}, 7 \mathrm{H}), 7.48-7.51(\mathrm{~m}, 2 \mathrm{H})$, $8.20 \& 8.26(\mathrm{~s}, 1 \mathrm{H}), 8.58 \& 9.90(\mathrm{~s}, 1 \mathrm{H}) ;{ }^{13} \mathrm{C} \mathrm{NMR}\left(150 \mathrm{MHz}, \mathrm{CDCl}_{3}\right): \boldsymbol{\delta}=37.4,43.7,52.2$, 53.4, 63.0, 120.2, 124.8, 128.2, 128.9, 129.1, 129.4, 136.1, 137.2, 161.9, 166.9; ESI HRMS exact mass calcd. for $\left(\mathrm{C}_{19} \mathrm{H}_{21} \mathrm{~N}_{3} \mathrm{O}_{2}+\mathrm{Na}\right)^{+}$requires $\mathrm{m} / \mathrm{z} 346.1526$, found $\mathrm{m} / \mathrm{z} 346.1506$.

4b: white solid; yield: $70 \% ;[\alpha]_{\mathrm{D}}{ }^{20}=-42.1(\mathrm{c}=0.12, \mathrm{MeOH}) ; \mathrm{mp} 198.0-202.0{ }^{\circ} \mathrm{C} ;{ }^{1} \mathrm{H}$ NMR (600 MHz, DMSO): $\boldsymbol{\delta}=2.71 \& 2.83(\mathrm{td}, J=3.42,11.76 \mathrm{~Hz}, 1 \mathrm{H}), 2.90(\mathrm{~s}, 3 \mathrm{H}), 3.05 \&$ $3.10(\mathrm{dd}, J=4.56,12.72 \mathrm{~Hz}, 1 \mathrm{H}), 3.17 \& 3.66(\mathrm{td}, J=3.6,12.66 \mathrm{~Hz}, 1 \mathrm{H}), 3.54 \& 3.58(\mathrm{~d}, J=$ $11.58 \mathrm{~Hz}, 1 \mathrm{H}), 3.82 \& 4.20(\mathrm{~d}, J=12.24 \mathrm{~Hz}, 1 \mathrm{H}), 4.12 \& 4.26(\mathrm{~d}, J=12.48 \mathrm{~Hz}, 1 \mathrm{H}), 4.74 \&$ $5.01(\mathrm{~s}, 1 \mathrm{H}), 7.10(\mathrm{t}, J=7.52 \mathrm{~Hz}, 1 \mathrm{H}), 7.34$ (t, $J=7.52 \mathrm{~Hz}, 2 \mathrm{H}), 7.56 \& 7.60(\mathrm{~d}, J=7.68 \mathrm{~Hz}$, $2 \mathrm{H}), 8.18 \& 8.23(\mathrm{~s}, 1 \mathrm{H}), 10.01 \& 10.05(\mathrm{~s}, 1 \mathrm{H}) ;{ }^{13} \mathrm{C}$ NMR $(150 \mathrm{MHz}, \mathrm{DMSO}): \boldsymbol{\delta}=35.3$, 43.3, 45.8, 47.0, 51.0, 120.4, 124.3, 129.2, 138.8, 163.4, 167.2; ESI HRMS exact mass calcd. for $\left(\mathrm{C}_{13} \mathrm{H}_{17} \mathrm{~N}_{3} \mathrm{O}_{4} \mathrm{~S}+\mathrm{Na}\right)^{+}$requires $\mathrm{m} / \mathrm{z} 334.0832$, found $\mathrm{m} / \mathrm{z} 334.0835$. 
4c: white solid; yield: $70 \% ;[\alpha]_{\mathrm{D}}{ }^{20}=-62.0\left(\mathrm{c}=0.11, \mathrm{CHCl}_{3}\right) ; \operatorname{mp} 211.0-212.0{ }^{\circ} \mathrm{C} ;{ }^{1} \mathrm{H} \mathrm{NMR}$ $\left(600 \mathrm{MHz}, \mathrm{CDCl}_{3} / \mathrm{CD}_{3} \mathrm{OD}\right): \boldsymbol{\delta}=2.44-2.51(\mathrm{~m}, 1 \mathrm{H}), 2.61 \& 2.71(\mathrm{dd}, J=12.48,4.32 \mathrm{~Hz}$, 1H), $3.66 \& 3.72(\mathrm{~d}, J=11.52 \mathrm{~Hz}, 1 \mathrm{H}), 3.80-3.85(\mathrm{~m}, 2 \mathrm{H}), 4.38-4.41(\mathrm{~m}, 1 \mathrm{H}), 5.09$ (s, 1H), 7.12 - $7.17(\mathrm{~m}, 1 \mathrm{H}), 7.31-7.36(\mathrm{~m}, 2 \mathrm{H}), 7.51-7.57$ (m, 4H), $7.62-7.66(\mathrm{~m}, 1 \mathrm{H}), 7.77$ $(\mathrm{d}, J=7.56 \mathrm{~Hz}, 2 \mathrm{H}), 8.12 \& 8.08(\mathrm{~s}, 1 \mathrm{H}) ;{ }^{13} \mathrm{C} \mathrm{NMR}\left(150 \mathrm{MHz}, \mathrm{CDCl}_{3} / \mathrm{CD}_{3} \mathrm{OD}\right): \boldsymbol{\delta}=37.7$, $43.2,45.6,51.1,120.5,124.6,127.4,128.7,129.2,133.3,135.5,137.4,162.8,165.8$; ESI HRMS exact mass calcd. for $\left(\mathrm{C}_{18} \mathrm{H}_{19} \mathrm{~N}_{3} \mathrm{O}_{4} \mathrm{~S}+\mathrm{Na}\right)^{+}$requires $\mathrm{m} / \mathrm{z} 396.0988$, found $\mathrm{m} / \mathrm{z}$ 396.0972 .

4d: white solid; yield: $86 \%$; $[\alpha]_{\mathrm{D}}{ }^{20}=-61.2\left(\mathrm{c}=0.15, \mathrm{CHCl}_{3}\right) ; \mathrm{mp} 96.0-100.0{ }^{\circ} \mathrm{C} ;{ }^{1} \mathrm{H} \mathrm{NMR}$ $\left(600 \mathrm{MHz}, \mathrm{CDCl}_{3}\right): \boldsymbol{\delta}=2.43(\mathrm{~s}, 3 \mathrm{H}), 2.37-2.48(\mathrm{~m}, 1 \mathrm{H}), 2.52 \& 2.57(\mathrm{dd}, J=12.6,3.66 \mathrm{~Hz}$, 1H), $3.61(\mathrm{~m}, 1 \mathrm{H}), 3.80(\mathrm{~d}, J=9.0 \mathrm{~Hz}, 1 \mathrm{H}), 4.38(\mathrm{~m}, 1 \mathrm{H}), 4.26 \& 5.14(\mathrm{~s}, 1 \mathrm{H}), 7.12 \& 7.16(\mathrm{t}$, $J=7.44 \mathrm{~Hz}, 1 \mathrm{H}), 7.29-7.36(\mathrm{~m}, 4 \mathrm{H}), 7.51 \& 7.66(\mathrm{~d}, J=7.62 \mathrm{~Hz}, 2 \mathrm{H}), 7.66(\mathrm{~d}, J=7.44 \mathrm{~Hz}$, 2H), $7.97 \& 8.33(\mathrm{~s}, 1 \mathrm{H}), 8.17(\mathrm{~s}, 1 \mathrm{H}) ;{ }^{13} \mathrm{C} \mathrm{NMR}\left(150 \mathrm{MHz}, \mathrm{CDCl}_{3}\right): \boldsymbol{\delta}=21.6,37.7,43.2$, $45.8,51.4,120.2,124.8,127.8,129.0,130.0,132.6,137.2,144.4,162.3,165.0$; ESI HRMS exact mass calcd. for $\left(\mathrm{C}_{19} \mathrm{H}_{21} \mathrm{~N}_{3} \mathrm{O}_{4} \mathrm{~S}+\mathrm{Na}\right)^{+}$requires $\mathrm{m} / \mathrm{z} 410.1145$, found $\mathrm{m} / \mathrm{z} 410.1139$.

4e: white solid; yield: $90 \%$; $[\alpha]_{\mathrm{D}}{ }^{20}=-48.0\left(\mathrm{c}=0.10, \mathrm{CHCl}_{3}\right) ; \mathrm{mp} 212.0-214.0{ }^{\circ} \mathrm{C} ;{ }^{1} \mathrm{H} \mathrm{NMR}$ $\left(600 \mathrm{MHz}, \mathrm{CDCl}_{3}\right): \boldsymbol{\delta}=1.34(\mathrm{~s}, 9 \mathrm{H}), 2.43 \& 2.50(\mathrm{td}, J=3.06,11.52 \mathrm{~Hz}, 1 \mathrm{H}), 2.54 \& 2.60$ (dd, $J=3.66,12.66 \mathrm{~Hz}, 1 \mathrm{H}), 3.61-3.64(\mathrm{~m}, 1 \mathrm{H}), 3.80-3.84(\mathrm{~m}, 1 \mathrm{H}), 4.39-4.43(\mathrm{~m}, 2 \mathrm{H})$, $4.27 \& 5.16$ (brs, 1H), $7.12 \& 7.16(\mathrm{~d}, J=7.32 \mathrm{~Hz}, 1 \mathrm{H}), 7.31 \& 7.35(\mathrm{~d}, J=7.92 \mathrm{~Hz}, 2 \mathrm{H})$, $7.52-7.63(\mathrm{~m}, 4 \mathrm{H}), 7.70(\mathrm{~d}, J=8.28 \mathrm{~Hz}, 2 \mathrm{H}), 7.97 \& 8.35(\mathrm{~s}, 1 \mathrm{H}), 8.18(\mathrm{~s}, 1 \mathrm{H}),{ }^{13} \mathrm{C} \mathrm{NMR}$ (150 MHz, $\left.\mathrm{CDCl}_{3} / \mathrm{CD}_{3} \mathrm{OD}\right): \boldsymbol{\delta}=30.9,35.2,37.7,43.3,45.7,51.3,57.1,120.3,124.7,126.3$, 127.5, 128.9, 129.0, 132.4, 137.4, 157.3, 162.6; ESI HRMS exact mass calcd. for $\left(\mathrm{C}_{22} \mathrm{H}_{27} \mathrm{~N}_{3} \mathrm{O}_{4} \mathrm{~S}+\mathrm{Na}\right)^{+}$requires $\mathrm{m} / \mathrm{z} 452.1614$, found $\mathrm{m} / \mathrm{z} 452.1619$.

\section{Scheme for the synthesis of $5 a$ and $5 b$ :}




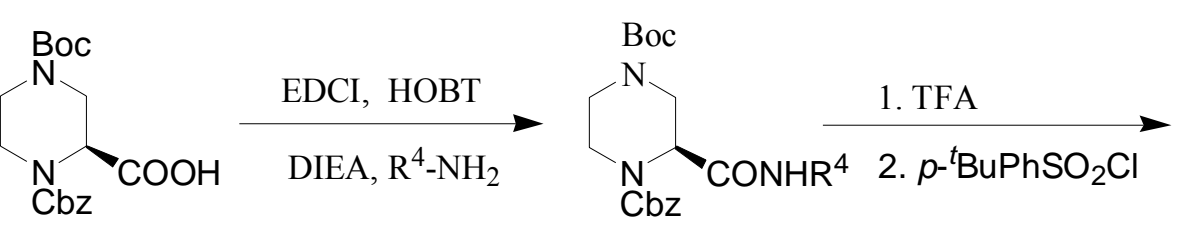

D

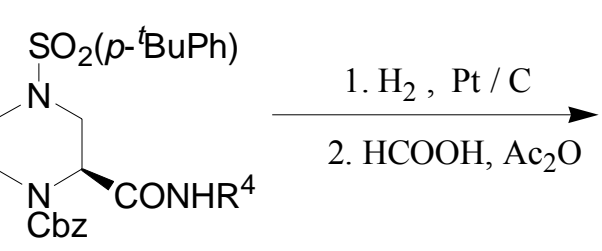

$$
\begin{array}{ll}
\text { a } \quad R^{4}=4-M e O P h \\
\text { b, } R^{4}=2-N a p h t h y l
\end{array}
$$

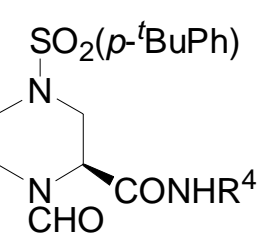

5

\section{Procedure for the synthesis of $5 \mathrm{a}$ and $5 \mathrm{~b}$ is similar to that for the synthesis of 4}

5a: white solid; yield: $80 \%$; $[\alpha]_{\mathrm{D}}{ }^{20}=-36.0(\mathrm{c}=0.10, \mathrm{MeOH}) ; \mathrm{mp} 126.0-127.0{ }^{\circ} \mathrm{C} ;{ }^{1} \mathrm{H} \mathrm{NMR}$ $\left(600 \mathrm{MHz}, \mathrm{CDCl}_{3}\right): \boldsymbol{\delta}=1.35(\mathrm{~s}, 9 \mathrm{H}), 2.40-2.45(\mathrm{~m}, 1 \mathrm{H}), 2.53 \& 2.53(\mathrm{dd}, J=12.6,3.84 \mathrm{~Hz}$, $1 \mathrm{H}), 3.60$ - $3.62(\mathrm{~m}, 1 \mathrm{H}), 3.79 \& 3.81(\mathrm{~s}, 3 \mathrm{H}), 3.82-3.84(\mathrm{~m}, 1 \mathrm{H}), 4.40(\mathrm{~d}, J=12.42 \mathrm{~Hz}, 1 \mathrm{H})$, $5.14 \& 4.25(\mathrm{~s}, 1 \mathrm{H}), 6.85 \& 6.88(\mathrm{~d}, J=8.76 \mathrm{~Hz}, 2 \mathrm{H}), 7.44(\mathrm{~d}, J=8.76 \mathrm{~Hz}, 1 \mathrm{H}), 7.52-7.58$ $(\mathrm{m}, 3 \mathrm{H}), 7.70(\mathrm{~d}, J=8.40 \mathrm{~Hz}, 2 \mathrm{H}), 7.84 \& 8.24(\mathrm{~s}, 1 \mathrm{H}), 8.17(\mathrm{~s}, 1 \mathrm{H}) ;{ }^{13} \mathrm{C} \mathrm{NMR}(150 \mathrm{MHz}$, $\left.\mathrm{CDCl}_{3}\right): \boldsymbol{\delta}=31.0,35.3,43.2,45.4,45.8,51.3,55.5,114.2,122.0,126.4,127.6,130.3,132.5$, 157.3, 162.3, 164.8; ESI HRMS exact mass calcd. for $\left(\mathrm{C}_{23} \mathrm{H}_{29} \mathrm{~N}_{3} \mathrm{O}_{5} \mathrm{~S}+\mathrm{Na}\right)^{+}$requires $\mathrm{m} / \mathrm{z}$ 482.1720 , found $\mathrm{m} / \mathrm{z} 482.1725$.

5b: white solid; yield: 70\%; $[\alpha]_{\mathrm{D}}{ }^{20}=-42.7(\mathrm{c}=0.10, \mathrm{MeOH}) ; \mathrm{mp} 129.0-130.0{ }^{\circ} \mathrm{C} ;{ }^{1} \mathrm{H}$ NMR $\left(600 \mathrm{MHz}, \mathrm{CDCl}_{3}\right): \boldsymbol{\delta}=1.33(\mathrm{~s}, 9 \mathrm{H}), 2.44-2.58(\mathrm{~m}, 2 \mathrm{H}), 3.63-3.80(\mathrm{~m}, 3 \mathrm{H}), 4.49-4.53(\mathrm{~m}$, $1 \mathrm{H}), 5.26 \& 4.48(\mathrm{~s}, 1 \mathrm{H}), 7.42-7.56(\mathrm{~m}, 5 \mathrm{H}), 7.66-7.87(\mathrm{~m}, 6 \mathrm{H}), 8.17 \& 8.18(\mathrm{~s}, 1 \mathrm{H}), 8.37$ $\& 8.64(\mathrm{~s}, 1 \mathrm{H}) ;{ }^{13} \mathrm{C}$ NMR $\left(150 \mathrm{MHz}, \mathrm{CDCl}_{3}\right): \boldsymbol{\delta}=21.0,31.0,35.2,43.2,45.8,45.8,60.4$, $120.9,125.6,126.1,126.3,126.5,126.6,127.7,128.6,131.9,132.5,134,157.2,162.5$, 171.1; ESI HRMS exact mass calcd. for $\left(\mathrm{C}_{26} \mathrm{H}_{29} \mathrm{~N}_{3} \mathrm{O}_{4} \mathrm{~S}+\mathrm{Na}\right)^{+}$requires $\mathrm{m} / \mathrm{z} 502.1771$, found $\mathrm{m} / \mathrm{z}$ 502.1759 .

\section{Scheme for the synthesis of $5 \mathrm{c}$ :}




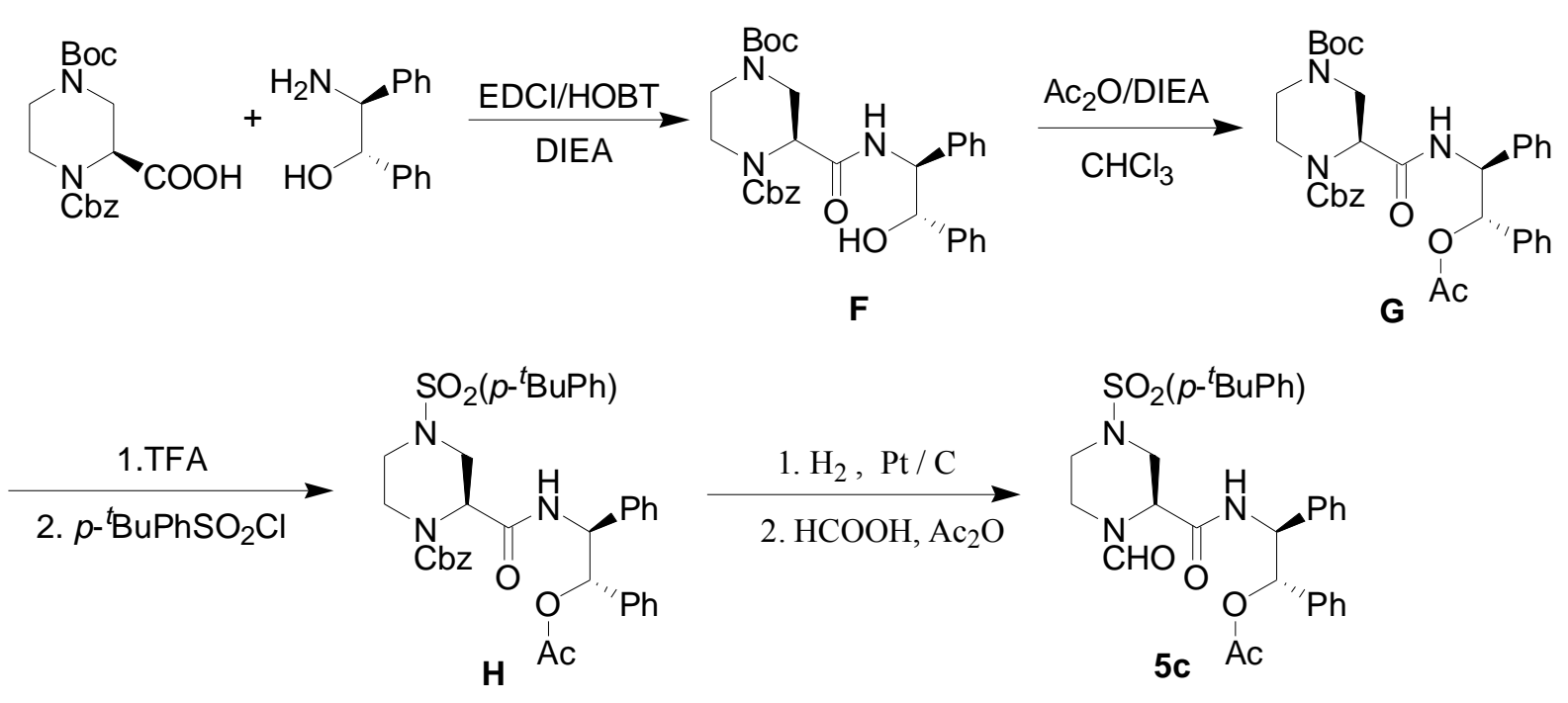

\section{Procedure for the synthesis of $F$ is similar to that for the synthesis of $B$}

Procedure for the synthesis of G: Acetic anhydride $(2 \mathrm{~mL}, 21.3 \mathrm{mmol})$ was added dropwise to a solution of $\mathbf{F}$ in chloroform. The mixture was refluxed for $5 \mathrm{~h}$ and then concentrated under reduced pressure. The residue was purified by column chromatography on silica gel (eluent: hexane/EtOAc $=10 / 1$ ) gave pure $\mathbf{G}$.

\section{Procedure for the synthesis of $5 \mathrm{c}$ from $\mathrm{G}$ is similar to that for the synthesis of 4 from $B$}

5c: white solid; yield: $81 \% ;[\alpha]_{\mathrm{D}}{ }^{20}=+38.0(\mathrm{c}=0.10, \mathrm{MeOH}) ; \operatorname{mp} 116.0-118.0{ }^{\circ} \mathrm{C} ;{ }^{1} \mathrm{H}$ $\operatorname{NMR}\left(600 \mathrm{MHz}, \mathrm{CDCl}_{3}\right): \boldsymbol{\delta}=1.35 \& 1.36(\mathrm{~s}, 9 \mathrm{H}), 2.03-2.06(\mathrm{~m}, 1 \mathrm{H}), 2.159 \& 2.164(\mathrm{~s}$, 3H), $2.24-2.48(\mathrm{~m}, 3 \mathrm{H}), 3.40 \& 4.10(\mathrm{~d}, J=13.68 \mathrm{~Hz}, 1 \mathrm{H}), 3.72 \& 3.73(\mathrm{~d}, J=11.16 \mathrm{~Hz}$, 1H), $3.94 \& 4.98(\mathrm{~s}, 1 \mathrm{H}), 4.20(\mathrm{~m}, 1 \mathrm{H}), 5.46 \& 5.54(\mathrm{dd}, J=8.34,5.64 \mathrm{~Hz} \& 8.64,3.66 \mathrm{~Hz}$, $1 \mathrm{H}), 6.08 \& 6.16(\mathrm{~d}, J=5.64 \mathrm{~Hz} \& 3.60 \mathrm{~Hz}, 1 \mathrm{H}), 7.21-7.72(\mathrm{~m}, 14 \mathrm{H}), 7.95 \& 8.02(\mathrm{~s}, 1 \mathrm{H})$; ${ }^{13} \mathrm{C}$ NMR $\left(150 \mathrm{MHz}, \mathrm{CDCl}_{3}\right): \boldsymbol{\delta}=20.8,31.0,35.3,42.5,45.4,50.6,56.2,58.3,60.4,125.9$, $126.3,126.6,126.9,127.0,127.1,127.7,128.0,128.2,128.4,128.6,128.7,131.3,137.4$, 157.9, 161.5, 166.4, 169.8; ESI HRMS exact mass calcd. for $\left(\mathrm{C}_{32} \mathrm{H}_{37} \mathrm{~N}_{3} \mathrm{O}_{6} \mathrm{~S}+\mathrm{Na}\right)^{+}$requires $\mathrm{m} / \mathrm{z} 614.2295$, found $\mathrm{m} / \mathrm{z} 614.2291$.

\section{Procedure for the synthesis of $5 \mathrm{~d}$ and $5 \mathrm{e}$ is similar to that for the synthesis of $5 \mathrm{c}$}

5d: white solid; yield: $86 \%$; $[\alpha]_{\mathrm{D}}{ }^{20}=+14.7(\mathrm{c}=0.10, \mathrm{MeOH}) ; \operatorname{mp} 105.0-107.0{ }^{\circ} \mathrm{C} ;{ }^{1} \mathrm{H}$ $\operatorname{NMR}\left(600 \mathrm{MHz}, \mathrm{CDCl}_{3}\right): \boldsymbol{\delta}=1.35 \& 1.36(\mathrm{~s}, 9 \mathrm{H}), 2.09 \& 2.13(\mathrm{~s}, 3 \mathrm{H}), 2.20-2.54(\mathrm{~m}, 2 \mathrm{H})$, $3.00-3.01(\mathrm{~m}, 1 \mathrm{H}), 3.39 \& 4.18(\mathrm{~d}, J=13.86 \mathrm{~Hz}, 1 \mathrm{H}), 3.68(\mathrm{~d}, J=11.16 \mathrm{~Hz}, 1 \mathrm{H}), 4.24-$ $4.30(\mathrm{~m}, 1 \mathrm{H}), 4.91 \& 4.05(\mathrm{~s}, 1 \mathrm{H}), 5.42-5.46(\mathrm{~m}, 1 \mathrm{H}), 6.10 \& 6.17(\mathrm{~d}, J=5.40 \mathrm{~Hz}, 1 \mathrm{H})$, 
$7.11-7.18(\mathrm{~m}, 4 \mathrm{H}), 7.28-7.35(\mathrm{~m}, 6 \mathrm{H}), 7.52-7.69(\mathrm{~m}, 4 \mathrm{H}), 8.0(\mathrm{~s}, 1 \mathrm{H}) ;{ }^{13} \mathrm{C}$ NMR $(150$ $\left.\mathrm{MHz}, \mathrm{CDCl}_{3}\right): \delta=21.0,31.0,35.2,42.8,44.8,45.7,50.9,57.6,60.4,126.3,126.4,127.1$, 127.6, 127.9, 128.2, 128.4, 128.5, 132.4, 136.8, 157.4, 162.0, 165.9, 170.7; ESI HRMS exact mass calcd. for $\left(\mathrm{C}_{32} \mathrm{H}_{37} \mathrm{~N}_{3} \mathrm{O}_{6} \mathrm{~S}+\mathrm{Na}\right)^{+}$requires $\mathrm{m} / \mathrm{z}$ 614.2295, found $\mathrm{m} / \mathrm{z}$ 614.2290.

5e: white solid; yield: $76 \% ;[\alpha]_{\mathrm{D}}{ }^{20}=+43.8(\mathrm{c}=0.10, \mathrm{MeOH}) ; \operatorname{mp} 222.0-223.0{ }^{\circ} \mathrm{C} ;{ }^{1} \mathrm{H}$ NMR (600 MHz, $\left.\mathrm{CDCl}_{3}\right): \boldsymbol{\delta}=1.35(\mathrm{~s}, 9 \mathrm{H}), 2.04 \& 2.10(\mathrm{~s}, 3 \mathrm{H}), 2.20-2.41(\mathrm{~m}, 2 \mathrm{H}), 3.01-$ $4.19(\mathrm{~m}, 4 \mathrm{H}), 5.00 \& 3.9(\mathrm{~s}, 1 \mathrm{H}), 5.47-5.49(\mathrm{~m}, 1 \mathrm{H}), 6.21 \& 6.18(\mathrm{~d}, J=6.72 \mathrm{~Hz}, 1 \mathrm{H}), 7.15$ $-7.18(\mathrm{~m}, 2 \mathrm{H}), 7.26-7.36(\mathrm{~m}, 8 \mathrm{H}), 7.53-7.57(\mathrm{~m}, 2 \mathrm{H}), 7.65-7.68(\mathrm{~m}, 2 \mathrm{H}), 8.01 \& 8.05(\mathrm{~s}$, $1 \mathrm{H}) ;{ }^{13} \mathrm{C}$ NMR $\left(150 \mathrm{MHz}, \mathrm{CDCl}_{3}\right): \boldsymbol{\delta}=21.0,31.1,35.3,36.7,42.7,45.2,46.0,50.7,57.6$, $126.4,126.5,127.3,127.6,127.8,128.0,128.2$, 128.5, 131.6, 136.7, 157.6, 161.9, 166.0, 170.1; ESI HRMS exact mass calcd. for $\left(\mathrm{C}_{32} \mathrm{H}_{37} \mathrm{~N}_{3} \mathrm{O}_{6} \mathrm{~S}+\mathrm{Na}\right)^{+}$requires $\mathrm{m} / \mathrm{z}$ 614.2295, found $\mathrm{m} / \mathrm{z} 614.2301$.

\section{General procedure for the synthesis of imines 6a - s:}

A mixture of $\mathrm{NaHCO}_{3}(50 \mathrm{mmol})$, amine $(10 \mathrm{mmol})$, ketone $(10 \mathrm{mmol})$ and activated molecular $4 \AA$ sieves $(8.0 \mathrm{~g})$ in anhydrous toluene $(10 \mathrm{~mL})$ was heated at $80{ }^{\circ} \mathrm{C}$ for $12 \mathrm{~h}$ under an argon atmosphere. The mixture was filtered through celite. The filtrate was then evaporated in vacuo and the product was crystallized from appropriate solvents or purified by distillation to give pure imine.

6h: Light yellow oil; Yield: 61\%; ${ }^{1} \mathrm{H}$ NMR $\left(300 \mathrm{MHz}, \mathrm{CDCl}_{3}\right): \boldsymbol{\delta}=1.0(\mathrm{t}, J=7.68 \mathrm{~Hz}, 3 \mathrm{H})$, $1.17-1.92(\mathrm{~m}, 10 \mathrm{H}), 2.37(\mathrm{~m}, 1 \mathrm{H}), 2.45$ (q, $J=7.29 \mathrm{~Hz}, 2 \mathrm{H}), 6.65$ (d, $J=7.32 \mathrm{~Hz}, 2 \mathrm{H}), 6.99$ $(\mathrm{t}, 1 \mathrm{H}), 7.26(\mathrm{t}, J=7.68 \mathrm{~Hz}, 2 \mathrm{H})$; minor isomer: 2.14 (q, $J=7.68 \mathrm{~Hz}, 2 \mathrm{H}) ;{ }^{13} \mathrm{C} \mathrm{NMR}(150$ $\left.\mathrm{MHz}, \mathrm{CDCl}_{3}\right): \boldsymbol{\delta}=11.8,25.2,26.4,28.6,30.8,46.0,119.2,122.4,128.7,151.7,179.7$; ESI HRMS exact mass calcd. for $\mathrm{C}_{15} \mathrm{H}_{22} \mathrm{~N}$ requires $\mathrm{m} / \mathrm{z} 216.1747$, found $\mathrm{m} / \mathrm{z} 216.1736$.

6j: Light yellow solid; Yield: $70 \%$; mp $69-72{ }^{\circ} \mathrm{C}$; a $15 / 1$ mixture of $E / Z$ isomers; ${ }^{1} \mathrm{H}$ NMR $\left(300 \mathrm{MHz}, \mathrm{CDCl}_{3}\right): \delta=1.08(\mathrm{t}, J=7.68 \mathrm{~Hz}, 3 \mathrm{H}), 2.64(\mathrm{q}, J=7.65 \mathrm{~Hz}, 2 \mathrm{H}), 6.78(\mathrm{~d}, J=8.4$ $\mathrm{Hz}, 2 \mathrm{H}), 7.08-7.16(\mathrm{~m}, 3 \mathrm{H}), 7.35(\mathrm{t}, J=7.89 \mathrm{~Hz}, 2 \mathrm{H}), 7.91-7.96(\mathrm{~m}, 2 \mathrm{H})$; minor isomer: $\boldsymbol{\delta}$ $=1.23(\mathrm{t}, J=7.38 \mathrm{~Hz}, 3 \mathrm{H}), 2.77(\mathrm{q}, J=7.50 \mathrm{~Hz}, 2 \mathrm{H}) ;{ }^{13} \mathrm{C} \mathrm{NMR}\left(150 \mathrm{MHz}, \mathrm{CDCl}_{3}\right): \boldsymbol{\delta}=12.9$, 
23.4, 115.4, 115.5, 119.1, 123.1, 129.0, 129.8, 134.2, 151.4, 163.4, 165.0, 169.5; ESI HRMS exact mass calcd. for $\mathrm{C}_{15} \mathrm{H}_{15} \mathrm{FN}$ requires $\mathrm{m} / \mathrm{z} 228.1183$, found $\mathrm{m} / \mathrm{z} 228.1176$.

6k: Light yellow solid; Yield: $75 \%$; mp $57-59{ }^{\circ} \mathrm{C}$; a $12 / 1$ mixture of $E / Z$ isomers; ${ }^{1} \mathrm{H}$ NMR $\left(300 \mathrm{MHz}, \mathrm{CDCl}_{3}\right): \delta=1.07(\mathrm{t}, J=7.68 \mathrm{~Hz}, 3 \mathrm{H}), 2.64(\mathrm{q}, J=7.68 \mathrm{~Hz}, 2 \mathrm{H}), 6.78(\mathrm{~d}, J=8.10$ Hz, 2H), 7.09 (t, $J=7.44 \mathrm{~Hz}, 1 \mathrm{H}), 7.35$ (t, $J=7.72 \mathrm{~Hz}, 2 \mathrm{H}), 7.42$ (d, $J=6.75 \mathrm{~Hz}, 2 \mathrm{H}), 7.87$ (d, $J=6.72 \mathrm{~Hz}, 2 \mathrm{H})$; minor isomer: $\delta=1.23(\mathrm{t}, J=7.26 \mathrm{~Hz}, 3 \mathrm{H}), 2.77$ (q, $J=7.47 \mathrm{~Hz}, 2 \mathrm{H}$ ); ${ }^{13} \mathrm{C} \mathrm{NMR}\left(150 \mathrm{MHz}, \mathrm{CDCl}_{3}\right): \boldsymbol{\delta}=12.87,23.4,119.0,120.8,123.2,128.7,129.0,129.4,136.5$, 151.3, 169.6; ESI HRMS exact mass calcd. for $\mathrm{C}_{15} \mathrm{H}_{15} \mathrm{ClN}$ requires $\mathrm{m} / \mathrm{z} 244.0888$, found $\mathrm{m} / \mathrm{z}$ 244.0882 .

61: Light yellow solid; Yield: $75 \%$; mp $37-39{ }^{\circ} \mathrm{C}$; a $14 / 1$ mixture of $E / Z$ isomers; ${ }^{1} \mathrm{H}$ NMR $\left(300 \mathrm{MHz}, \mathrm{CDCl}_{3}\right): \delta=1.07(\mathrm{t}, J=7.68 \mathrm{~Hz}, 3 \mathrm{H}), 2.63(\mathrm{q}, J=7.68 \mathrm{~Hz}, 2 \mathrm{H}), 6.77(\mathrm{~d}, J=7.29$ $\mathrm{Hz}, 2 \mathrm{H}), 7.10$ (t, $J=7.38 \mathrm{~Hz}, 1 \mathrm{H}), 7.35$ (t, $J=7.68 \mathrm{~Hz}, 2 \mathrm{H}), 7.58(\mathrm{~d}, J=8.58 \mathrm{~Hz}, 2 \mathrm{H}), 7.81$ $(\mathrm{d}, J=8.67 \mathrm{~Hz}, 2 \mathrm{H})$; minor isomer: $\boldsymbol{\delta}=1.22(\mathrm{t}, J=7.23 \mathrm{~Hz}, 3 \mathrm{H}), 2.77(\mathrm{q}, J=7.41 \mathrm{~Hz}, 2 \mathrm{H})$; ${ }^{13} \mathrm{C}$ NMR $\left(150 \mathrm{MHz}, \mathrm{CDCl}_{3}\right): \boldsymbol{\delta}=12.87,23.3,118.8,122.6,125.0,129.5,129.6,131.7,136.9$, 151.3, 169.7; ESI HRMS exact mass calcd. for $\mathrm{C}_{15} \mathrm{H}_{15} \mathrm{BrN}$ requires $\mathrm{m} / \mathrm{z} 288.0382$, found $\mathrm{m} / \mathrm{z}$ 288.0383 .

6m: Light yellow solid; Yield: $60 \%$; mp 47 - $48{ }^{\circ} \mathrm{C}$; a $11 / 1$ mixture of $E / Z$ isomers; ${ }^{1} \mathrm{H}$ NMR $\left(300 \mathrm{MHz}, \mathrm{CDCl}_{3}\right): \delta=1.08(\mathrm{t}, J=7.62 \mathrm{~Hz}, 3 \mathrm{H}), 2.41(\mathrm{~s}, 3 \mathrm{H}), 2.64(\mathrm{q}, J=7.64 \mathrm{~Hz}, 2 \mathrm{H})$, $6.79(\mathrm{~d}, J=8.40 \mathrm{~Hz}, 2 \mathrm{H}), 7.10(\mathrm{t}, J=7.45 \mathrm{~Hz}, 1 \mathrm{H}), 7.24-7.31(\mathrm{~m}, 2 \mathrm{H}), 7.34(\mathrm{t}, J=7.62 \mathrm{~Hz}$, $2 \mathrm{H}), 7.83(\mathrm{~d}, J=8.22 \mathrm{~Hz}, 2 \mathrm{H})$; minor isomer: $\delta=1.23(\mathrm{t}, J=7.39 \mathrm{~Hz}, 3 \mathrm{H}), 2.78(\mathrm{q}, J=7.45$ $\mathrm{Hz}, 2 \mathrm{H}) ;{ }^{13} \mathrm{C} \mathrm{NMR}\left(150 \mathrm{MHz}, \mathrm{CDCl}_{3}\right): \boldsymbol{\delta}=13.1,21.4,23.4,119.2,120.9,122.9,127.6,128.9$, 129.2, 135.3, 140.6, 151.8, 170.5; ESI HRMS exact mass calcd. for $\mathrm{C}_{16} \mathrm{H}_{18} \mathrm{~N}$ requires $\mathrm{m} / \mathrm{z}$ 224.1434 , found $\mathrm{m} / \mathrm{z} 224.1433$.

6n: Light yellow solid; Yield: 65\%; mp $51-52{ }^{\circ} \mathrm{C} ;{ }^{1} \mathrm{H}$ NMR $\left(300 \mathrm{MHz}, \mathrm{CDCl}_{3}\right): \boldsymbol{\delta}=1.09$ (t, $J=7.63 \mathrm{~Hz}, 3 \mathrm{H}), 2.63(\mathrm{q}, J=7.69 \mathrm{~Hz}, 2 \mathrm{H}), 3.87(\mathrm{~s}, 3 \mathrm{H}), 6.78(\mathrm{~d}, J=7.58 \mathrm{~Hz}, 2 \mathrm{H}), 6.96(\mathrm{~d}, J$ $=6.84 \mathrm{~Hz}, 2 \mathrm{H}), 6.96(\mathrm{t}, J=6.85 \mathrm{~Hz}, 1 \mathrm{H}), 7.34(\mathrm{t}, J=7.45 \mathrm{~Hz}, 2 \mathrm{H}), 7.90(\mathrm{~d}, J=6.84 \mathrm{~Hz}, 2 \mathrm{H})$; ${ }^{13} \mathrm{C}$ NMR $\left(150 \mathrm{MHz}, \mathrm{CDCl}_{3}\right): \boldsymbol{\delta}=13.1,23.3,55.4,113.8,119.3,122.8,129.0,129.3,130.6$, 151.9, 161.4, 169.8; ESI HRMS exact mass calcd. for $\mathrm{C}_{16} \mathrm{H}_{18} \mathrm{NO}$ requires $\mathrm{m} / \mathrm{z} 240.1383$, found $\mathrm{m} / \mathrm{z} 240.1378$. 
6q: Light yellow oil; Yield: 62\%; ${ }^{1} \mathrm{H}$ NMR (300 MHz, $\left.\mathrm{CDCl}_{3}\right): \boldsymbol{\delta}=1.02-1.07(\mathrm{~m}, 2 \mathrm{H}), 1.22$ $-1.28(\mathrm{~m}, 2 \mathrm{H}), 2.67(\mathrm{~m}, 1 \mathrm{H}), 6.56-7.57(\mathrm{~m}, 8 \mathrm{H}), 8.00-8.04(\mathrm{~m}, 2 \mathrm{H}) ;{ }^{13} \mathrm{C} \mathrm{NMR}(150 \mathrm{MHz}$, $\left.\mathrm{CDCl}_{3}\right): \boldsymbol{\delta}=9.5,11.6,17.1,120.1,122.6,128.0,128.5,132.7,138.1,151.1,172.9$; ESI HRMS exact mass calcd. for $\mathrm{C}_{16} \mathrm{H}_{16} \mathrm{~N}$ requires $\mathrm{m} / \mathrm{z} 222.1277$, found $\mathrm{m} / \mathrm{z} 222.1275$.

6r: Light yellow oil; Yield: 70\%; a 12/1 mixture of $E / Z$ isomers; ${ }^{1} \mathrm{H}$ NMR (300 $\left.\mathrm{MHz}, \mathrm{CDCl}_{3}\right)$ : $\boldsymbol{\delta}=0.77(\mathrm{t}, J=7.24 \mathrm{~Hz}, 3 \mathrm{H}), 1.19-1.25(\mathrm{~m}, 2 \mathrm{H}), 1.40-1.60(\mathrm{~m}, 2 \mathrm{H}), 2.64(\mathrm{t}, J=7.88 \mathrm{~Hz}$, $3 \mathrm{H}), 6.78(\mathrm{~d}, J=8.43 \mathrm{~Hz}, 2 \mathrm{H}), 7.07(\mathrm{t}, J=7.42 \mathrm{~Hz}, 1 \mathrm{H}), 7.34(\mathrm{t}, J=8.04 \mathrm{~Hz}, 2 \mathrm{H}), 7.44-$ $7.46(\mathrm{~m}, 3 \mathrm{H}), 7.89-7.92(\mathrm{~m}, 2 \mathrm{H})$; minor isomer: $\boldsymbol{\delta}=2.76(\mathrm{t}, J=7.88 \mathrm{~Hz}, 2 \mathrm{H}) ;{ }^{13} \mathrm{C} \mathrm{NMR}$ $\left(150 \mathrm{MHz}, \mathrm{CDCl}_{3}\right): \boldsymbol{\delta}=13.6,22.7,30.3,38.3,119.2,122.9,127.5,128.4,128.9,130.2,138.6$, 151.6, 169.9; ESI HRMS exact mass calcd. for $\mathrm{C}_{17} \mathrm{H}_{20} \mathrm{~N}$ requires $\mathrm{m} / \mathrm{z} 238.1590$, found $\mathrm{m} / \mathrm{z}$ 238.1592 .

6s: Light yellow oil; Yield: 55\%; a 8/1 mixture of $E / Z$ isomers; ${ }^{1} \mathrm{H}$ NMR (300 $\left.\mathrm{MHz}, \mathrm{CDCl}_{3}\right)$ : $\boldsymbol{\delta}=0.78(\mathrm{~d}, J=6.65 \mathrm{~Hz}, 6 \mathrm{H}), 1.86-1.93(\mathrm{~m}, 1 \mathrm{H}), 2.59(\mathrm{~d}, J=7.44 \mathrm{~Hz}, 2 \mathrm{H}), 6.78-6.79(\mathrm{~m}$, $2 \mathrm{H}), 7.04-7.11(\mathrm{~m}, 1 \mathrm{H}), 7.26-7.56(\mathrm{~m}, 5 \mathrm{H}), 7.84-7.98(\mathrm{~m}, 2 \mathrm{H})$; minor isomer: $\boldsymbol{\delta}=1.00(\mathrm{~d}$, $J=6.65 \mathrm{~Hz}, 6 \mathrm{H}), 2.68(\mathrm{~d}, J=7.44,2 \mathrm{H}) ;{ }^{13} \mathrm{C} \mathrm{NMR}\left(150 \mathrm{MHz}, \mathrm{CDCl}_{3}\right): \boldsymbol{\delta}=22.5,22.8,27.0$, 38.9, 119.5, 122.9, 127.6, 128.4, 128.8, 130.1, 139.1, 151.4, 169.8; ESI HRMS exact mass calcd for $\mathrm{C}_{17} \mathrm{H}_{20} \mathrm{~N}$ requires $\mathrm{m} / \mathrm{z} 238.1590$, found $\mathrm{m} / \mathrm{z} 238.1600$.

\section{General procedure for catalytic hydrosilylation of imines $6 a-s$.}

Under an argon atmosphere, trichlorosilane $(40 \mu \mathrm{L}, 0.4 \mathrm{mmol})$ was added dropwise to a stirred solution of imine $6(0.20 \mathrm{mmol})$ and catalyst $4 \mathrm{e}(7.9 \mathrm{mg}, 0.02 \mathrm{mmol})$ in anhydrous $\mathrm{CH}_{2} \mathrm{Cl}_{2}$ at $-20^{\circ} \mathrm{C}$. The mixture was allowed to stir at the same temperature for $48 \mathrm{~h}$. The reaction was quenched with a saturated aqueous solution of $\mathrm{NaHCO}_{3}(5 \mathrm{~mL})$ and was extracted with EtOAc. The combined extracts was washed with brine and dried over anhydrous $\mathrm{MgSO}_{4}$. Solvents were evaporated. The crude product was purified by column chromatography (silica gel, hexane/EtOAc) to afford pure amine 7. The ee values were determined by using established HPLC techniques with chiral stationary phases.

7a: Light yellow oil; Yield: 95\%, purification by flash chromatography (hexane/EtOAc = 98/2); The enantiomers were analyzed by HPLC using a chiral OD-H column (n-heptane $/ 2$-propanol $=99 / 1$, flow rate $=1.0 \mathrm{~mL} / \mathrm{min}$, wavelength $=254 \mathrm{~nm}$ ); minor 
enantiomer: $t_{R}=11.82 \min$, major enantiomer: $t_{R}=16.05 \mathrm{~min} ; 89 \%$ ee.

7b: Light yellow oil; Yield: 99\%, purification by flash chromatography (hexane/EtOAc = 95/5); The enantiomers were analyzed by HPLC using a chiral OD-H column (n-heptane $/ 2$-propanol $=85 / 15$, flow rate $=1.0 \mathrm{~mL} / \mathrm{min}$, wavelength $=254 \mathrm{~nm}$ ); minor enantiomer: $t_{R}=19.59$ min, major enantiomer: $t_{R}=24.74 \mathrm{~min} ; 90 \%$ ee.

7c: Light yellow solid; Yield: $81 \%$, purification by flash chromatography (hexane/EtOAc = 98/2); The enantiomers were analyzed by HPLC using a chiral OD-H column (n-heptane $/ 2$-propanol $=95 / 5$, flow rate $=1.0 \mathrm{~mL} / \mathrm{min}$, wavelength $=254 \mathrm{~nm}$ ); minor enantiomer: $t_{R}=11.42 \mathrm{~min}$, major enantiomer: $t_{R}=15.37 \mathrm{~min} ; 89 \%$ ee.

7d: Light yellow oil; Yield: 71\%, purification by flash chromatography (hexane/EtOAc = 98/2); The enantiomers were analyzed by HPLC using a chiral OD-H column (n-heptane $/ 2$-propanol $=99 / 1$, flow rate $=1.0 \mathrm{~mL} / \mathrm{min}$, wavelength $=254 \mathrm{~nm}$ ); minor enantiomer: $t_{R}=9.86$ min, major enantiomer: $t_{R}=12.11 \mathrm{~min} ; 85 \%$ ee.

7e: Yield: $63 \%$, purification by flash chromatography (2\% EtOAc in hexane); The enantiomers were analyzed by HPLC using a chiral OD-H column ( $n$-heptane/2-Propanol = $98 / 2$, flow rate $=1.0 \mathrm{~mL} / \mathrm{min}$, wavelength $=254 \mathrm{~nm})$; minor enantiomer: $t_{R}=14.93 \mathrm{~min}$, major enantiomer: $t_{R}=17.73 \mathrm{~min} ; 88 \%$ ee.

7f: Yield: $64 \%$, purification by flash chromatography (2\% EtOAc in hexane); The enantiomers were analyzed by HPLC using a chiral OD-H column ( $n$-heptane/2-Propanol = $98 / 2$, flow rate $=1.0 \mathrm{~mL} / \mathrm{min}$, wavelength $=254 \mathrm{~nm}$ ); minor enantiomer: $t_{R}=18.69 \mathrm{~min}$, major enantiomer: $\mathrm{t}_{\mathrm{R}}=24.66 \mathrm{~min} ; 85 \%$ ee.

7g: Yield: $86 \%$, purification by flash chromatography (2\% EtOAc in hexane); The enantiomers were analyzed by HPLC using a chiral OJ-H column ( $n$-heptane/2-Propanol =

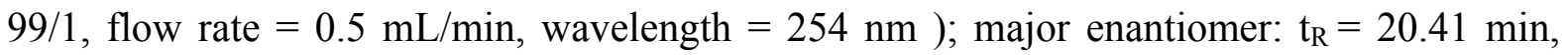
minor enantiomer: $\mathrm{t}_{\mathrm{R}}=22.62 \mathrm{~min} ; 82 \%$ ee.

7h: Light yellow oil; Yield: 84\%, purification by flash chromatography (hexane/EtOAc $=$ 98/2); $[\boldsymbol{\alpha}]_{\mathrm{D}}{ }^{20}=+10.53\left(c=0.38\right.$, EtOAc); ${ }^{1} \mathrm{H} \mathrm{NMR}\left(300 \mathrm{MHz}, \mathrm{CDCl}_{3}\right): \boldsymbol{\delta}=0.93(\mathrm{t}, J=7.36$ $\mathrm{Hz}, 3 \mathrm{H}), 1.11-1.22(\mathrm{~m}, 5 \mathrm{H}), 1.65-1.77$ (m, 8H), $3.10-3.16(\mathrm{~m}, 1 \mathrm{H}), 3.44$ (brs, 1H), 6.56 - 6.65 (m, 3H), 7.12 - 7.26 (m, 2H); $\left.{ }^{13} \mathrm{C} \mathrm{NMR} \mathrm{(150} \mathrm{MHz,} \mathrm{CDCl}_{3}\right): \boldsymbol{\delta}=10.8,24.7,26.5,26.6$, 26.7, 29.0, 29.5, 41.6, 59.2, 112.7, 116.2, 129.3, 148.9; ESI HRMS exact mass calcd. for $\mathrm{C}_{15} \mathrm{H}_{24} \mathrm{~N}$ requires $\mathrm{m} / \mathrm{z} 218.1903$, found $\mathrm{m} / \mathrm{z} 218.1912$.

Due to the difficulty of the separation of the enantiomers by HPLC with regular chiral 
columns, 7 h was $N$-formylated and the resulted $N$-Formyl-7h enantiomers were successfully analyzed using a chiral OJ-H column (n-heptane/2-propanol $=98 / 2$, flow rate $=1.0 \mathrm{~mL} / \mathrm{min}$, wavelength $=254 \mathrm{~nm}$ ); minor enantiomer: $t_{R}=8.28$ min, major enantiomer: $t_{R}=9.98$ min; $84 \%$ ee.

$\boldsymbol{N}$-Formyl-7h: ${ }^{1} \mathrm{H}$ NMR $\left(300 \mathrm{MHz}, \mathrm{CDCl}_{3}\right): \boldsymbol{\delta}=0.91$ (t, $\left.J=7.32 \mathrm{~Hz}, 3 \mathrm{H}\right), 1.03-1.25$ (m, 6H), $1.72-2.04(\mathrm{~m}, 7 \mathrm{H}), 4.09(\mathrm{dt}, J=3.09,10.68 \mathrm{~Hz}, 1 \mathrm{H}), 7.16(\mathrm{~d}, J=7.98 \mathrm{~Hz}, 2 \mathrm{H}), 7.21-$ $7.42(\mathrm{~m}, 3 \mathrm{H}), 8.43(\mathrm{~s}, 1 \mathrm{H})$.

7i: Yield: $92 \%$, purification by flash chromatography (2\% EtOAc in hexane); The enantiomers were analyzed by HPLC using a chiral OD-H column ( n-heptane/2-Propanol = 99/1, flow rate $=1.0 \mathrm{~mL} / \mathrm{min}$, wavelength $=254 \mathrm{~nm} \mathrm{);} \mathrm{minor} \mathrm{enantiomer:} t_{R}=8.15 \mathrm{~min}$, major enantiomer: $t_{R}=10.27 \mathrm{~min}$; $94 \%$ ee.

7j: Light yellow oil; Yield: 87\%, purification by flash chromatography (hexane/EtOAc $=$ 98/2); $[\alpha]_{\mathrm{D}}{ }^{20}=-24.85(c=0.676, \mathrm{EtOAc}) ;{ }^{1} \mathrm{H}$ NMR $\left(300 \mathrm{MHz}, \mathrm{CDCl}_{3}\right): \boldsymbol{\delta}=0.95(\mathrm{t}, J=7.41$ Hz, 3H), $1.72-1.89$ (m, 2H), $4.06(\mathrm{~s}, 1 \mathrm{H}), 4.21$ (t, $J=6.54 \mathrm{~Hz}, 1 \mathrm{H}), 6.50(\mathrm{~d}, J=8.26 \mathrm{~Hz}$, 2H), 6.67 (t, $J=7.28 \mathrm{~Hz}, 1 \mathrm{H}), 7.01(\mathrm{t}, J=8.66 \mathrm{~Hz}, 2 \mathrm{H}), 7.10(\mathrm{t}, J=7.68 \mathrm{~Hz}, 2 \mathrm{H}), 7.28-$ $7.33(\mathrm{~m}, 2 \mathrm{H}) ;{ }^{13} \mathrm{C} \mathrm{NMR}\left(150 \mathrm{MHz}, \mathrm{CDCl}_{3}\right): \boldsymbol{\delta}=10.8,31.8,59.2,113.3,115.3,115.4,117.4$, 128.0, 129.2, 139.7, 147.4, 161.0, 162.7; ESI HRMS exact mass calcd. for $\mathrm{C}_{15} \mathrm{H}_{17} \mathrm{FN}$ requires $\mathrm{m} / \mathrm{z} 230.1340$, found $\mathrm{m} / \mathrm{z} 230.1345$.

The enantiomers were analyzed by HPLC using a chiral OD-H column (n-heptane/2-propanol $=99 / 1$, flow rate $=1.0 \mathrm{~mL} / \mathrm{min}$, wavelength $=254 \mathrm{~nm}$ ); minor enantiomer: $t_{R}=9.13 \mathrm{~min}$, major enantiomer: $t_{R}=13.30 \mathrm{~min}$; $95 \%$ ee.

7k: Light yellow oil; Yield: 83\%, purification by flash chromatography (hexane/EtOAc $=$ 98/2); $[\boldsymbol{\alpha}]_{\mathrm{D}}{ }^{20}=-7.89(c=0.57, \mathrm{EtOAc}) ;{ }^{1} \mathrm{H} \mathrm{NMR}\left(300 \mathrm{MHz}, \mathrm{CDCl}_{3}\right): \boldsymbol{\delta}=0.95(\mathrm{t}, J=7.41 \mathrm{~Hz}$, $3 \mathrm{H}), 1.74-1.86(\mathrm{~m}, 2 \mathrm{H}), 4.05(\mathrm{~s}, 1 \mathrm{H}), 4.21(\mathrm{t}, J=6.64 \mathrm{~Hz}, 1 \mathrm{H}), 6.48(\mathrm{~d}, J=7.71 \mathrm{~Hz}, 2 \mathrm{H})$, $6.65(\mathrm{t}, J=7.31 \mathrm{~Hz}, 1 \mathrm{H}), 7.09(\mathrm{t}, J=7.46 \mathrm{~Hz}, 2 \mathrm{H}), 7.26-7.34(\mathrm{~m}, 4 \mathrm{H}) ;{ }^{13} \mathrm{C} \mathrm{NMR}(150 \mathrm{MHz}$, $\left.\mathrm{CDCl}_{3}\right): \boldsymbol{\delta}=10.8,31.7,59.2,113.3,117.5,127.9,128.7,129.2,132.5,142.6,147.3$.

The enantiomers were analyzed by HPLC using a chiral OD-H column (n-heptane/2-propanol $=99 / 1$, flow rate $=1.0 \mathrm{~mL} / \mathrm{min}$, wavelength $=254 \mathrm{~nm}$ ); minor enantiomer: $t_{R}=9.95 \mathrm{~min}$, major enantiomer: $t_{R}=15.12 \min ; 94 \%$ ee. 
71: Light yellow oil; Yield: $89 \%$, purification by flash chromatography (hexane/EtOAc $=$ 98/2); $[\boldsymbol{\alpha}]_{\mathrm{D}}{ }^{20}=-2.31\left(c=0.736\right.$, EtOAc); ${ }^{1} \mathrm{H}$ NMR $\left(300 \mathrm{MHz}, \mathrm{CDCl}_{3}\right): \boldsymbol{\delta}=0.95(\mathrm{t}, J=7.38$ Hz, 3H), $1.75-1.85$ (m, 2H), 4.05 (s, 1H), 4.18 (brs, 1H), 6.47 (d, J=7.68 Hz, 2H), 6.67 (t, $J=7.32 \mathrm{~Hz}, 1 \mathrm{H}), 7.08(\mathrm{t}, J=7.35 \mathrm{~Hz}, 2 \mathrm{H}), 7.24(\mathrm{t}, J=8.82 \mathrm{~Hz}, 2 \mathrm{H}), 7.43$ (d, $J=6.48 \mathrm{~Hz}$, $2 \mathrm{H}) ;{ }^{13} \mathrm{C} \mathrm{NMR}\left(150 \mathrm{MHz}, \mathrm{CDCl}_{3}\right): \boldsymbol{\delta}=10.8,31.7,59.2,113.3,117.4,120.5,128.3,129.2$, 131.6, 143.1, 147.2; ESI HRMS exact mass calcd. for $\mathrm{C}_{15} \mathrm{H}_{17} \mathrm{BrN}$ requires $\mathrm{m} / \mathrm{z} 290.0539$, found $\mathrm{m} / \mathrm{z} 290.0544$.

The enantiomers were analyzed by HPLC using a chiral OD-H column (n-heptane/2-propanol $=95 / 5$, flow rate $=1.0 \mathrm{~mL} / \mathrm{min}$, wavelength $=254 \mathrm{~nm}$ ); minor enantiomer: $t_{R}=7.72 \mathrm{~min}$, major enantiomer: $t_{R}=11.02 \mathrm{~min} ; 95 \%$ ee.

7m: Light yellow oil; Yield: $87 \%$, purification by flash chromatography (hexane/EtOAc $=$ 98/2); $[\boldsymbol{\alpha}]_{\mathrm{D}}{ }^{20}=-6.16\left(c=0.406\right.$, EtOAc); ${ }^{1} \mathrm{H}$ NMR $\left(300 \mathrm{MHz}, \mathrm{CDCl}_{3}\right): \boldsymbol{\delta}=0.96(\mathrm{t}, J=7.38$ $\mathrm{Hz}, 3 \mathrm{H}), 1.76-1.87(\mathrm{~m}, 2 \mathrm{H}), 2.33(\mathrm{~s}, 3 \mathrm{H}), 4.20(\mathrm{t}, J=6.70 \mathrm{~Hz}, 1 \mathrm{H}), 6.51(\mathrm{~d}, J=8.64 \mathrm{~Hz}$, $2 \mathrm{H}), 6.63(\mathrm{t}, J=7.30 \mathrm{~Hz}, 1 \mathrm{H}), 7.06-7.26(\mathrm{~m}, 6 \mathrm{H}) ;{ }^{13} \mathrm{C} \mathrm{NMR}\left(150 \mathrm{MHz}, \mathrm{CDCl}_{3}\right): \boldsymbol{\delta}=10.9$, 21.1, 31.7, 59.5, 113.2, 117.1, 126.4, 129.1, 129.2, 136.4, 140.9, 147.6; ESI HRMS exact mass calcd. for $\mathrm{C}_{16} \mathrm{H}_{20} \mathrm{~N}$ requires $\mathrm{m} / \mathrm{z} 226.1590$, found $\mathrm{m} / \mathrm{z} 226.1588$.

The enantiomers were analyzed by HPLC using a chiral OD-H column (n-heptane/2-propanol $=99 / 1$, flow rate $=1.0 \mathrm{~mL} / \mathrm{min}$, wavelength $=254 \mathrm{~nm}$ ); minor enantiomer: $t_{R}=6.71 \mathrm{~min}$, major enantiomer: $\mathrm{t}_{\mathrm{R}}=8.60 \mathrm{~min} ; 88 \%$ ee.

7n: Light yellow oil; Yield: $83 \%$, purification by flash chromatography (hexane/EtOAc $=$ 98/2); $[\boldsymbol{\alpha}]_{\mathrm{D}}{ }^{20}=-10.94\left(c=0.384\right.$, EtOAc); ${ }^{1} \mathrm{H}$ NMR $\left(300 \mathrm{MHz}, \mathrm{CDCl}_{3}\right): \boldsymbol{\delta}=0.95(\mathrm{t}, J=7.40$ Hz, 3H), $1.75-1.85$ (m, 2H), 3.79 (s, 3H), 4.19 (t, $J=6.68 \mathrm{~Hz}, 1 \mathrm{H}), 6.53$ (d, $J=7.68 \mathrm{~Hz}$, 2H), $6.64(\mathrm{t}, J=7.31 \mathrm{~Hz}, 1 \mathrm{H}), 6.87(\mathrm{~d}, J=8.62 \mathrm{~Hz}, 2 \mathrm{H}), 7.10(\mathrm{t}, J=7.49 \mathrm{~Hz}, 2 \mathrm{H}), 7.27(\mathrm{~d}, J$ $=8.61 \mathrm{~Hz}, 2 \mathrm{H}) ;{ }^{13} \mathrm{C} \mathrm{NMR}\left(150 \mathrm{MHz}, \mathrm{CDCl}_{3}\right): \boldsymbol{\delta}=10.8,31.7,55.2,59.1,113.3,113.9,117.1$, 127.5, 129.1, 135.9, 147.6, 158.5; ESI HRMS exact mass calcd. for $\left(\mathrm{C}_{16} \mathrm{H}_{19} \mathrm{NO}+\mathrm{Na}\right)^{+}$ requires $\mathrm{m} / \mathrm{z} 264.1359$, found $\mathrm{m} / \mathrm{z} 264.1361$.

The enantiomers were analyzed by HPLC using a chiral OD-H column (n-heptane/2-propanol $=99 / 1$, flow rate $=1.0 \mathrm{~mL} / \mathrm{min}$, wavelength $=254 \mathrm{~nm}$ ); minor enantiomer: $t_{R}=10.74 \mathrm{~min}$, 
major enantiomer: $t_{R}=13.92 \mathrm{~min} ; 90 \%$ ee.

7o: Light yellow oil; Yield: $88 \%$, purification by flash chromatography (hexane/EtOAc $=$ 98/2); The enantiomers were analyzed by HPLC using a chiral OD-H column (n-heptane $/ 2$-propanol $=99 / 1$, flow rate $=1.0 \mathrm{~mL} / \mathrm{min}$, wavelength $=254 \mathrm{~nm}$ ); minor enantiomer: $t_{R}=7.30$ min, major enantiomer: $t_{R}=9.17 \mathrm{~min} ; 90 \%$ ee.

7p: Light yellow oil; Yield: 75\%, purification by flash chromatography (hexane/EtOAc $=$ 98/2); The enantiomers were analyzed by HPLC using a chiral OD-H column (n-heptane $/ 2$-propanol $=99 / 1$, flow rate $=1.0 \mathrm{~mL} / \mathrm{min}$, wavelength $=254 \mathrm{~nm}$ ); minor enantiomer: $t_{R}=6.22 \mathrm{~min}$, major enantiomer: $t_{R}=7.26 \mathrm{~min} ; 92 \%$ ee.

7q: Light yellow oil; Yield: $85 \%$, purification by flash chromatography (hexane/EtOAc $=$ 98/2); $[\boldsymbol{\alpha}]_{\mathrm{D}}{ }^{20}=-80.10\left(c=0.412\right.$, EtOAc); ${ }^{1} \mathrm{H}$ NMR $\left(300 \mathrm{MHz}, \mathrm{CDCl}_{3}\right): \boldsymbol{\delta}=0.40-0.60(\mathrm{~m}$, 4H), $1.18-1.29(\mathrm{~m}, 1 \mathrm{H}), 3.65$ (d, $J=8.40 \mathrm{~Hz}, 1 \mathrm{H}), 4.41$ (brs, 1H), 6.48 (d, J=7.63 Hz, 2H), $6.64(\mathrm{t}, J=7.39 \mathrm{~Hz}, 1 \mathrm{H}), 7.09$ (t, $J=7.39 \mathrm{~Hz}, 2 \mathrm{H}), 7.25-7.43(\mathrm{~m}, 5 \mathrm{H}) ;{ }^{13} \mathrm{C} \mathrm{NMR}(150 \mathrm{MHz}$, $\left.\mathrm{CDCl}_{3}\right): \boldsymbol{\delta}=3.5,4.2,19.7,63.0,113.4,117.3,126.4,127.1,128.5,129.1,143.3,147.6$; ESI HRMS exact mass calcd. for $\left(\mathrm{C}_{16} \mathrm{H}_{17} \mathrm{~N}+\mathrm{Na}\right)^{+}$requires $\mathrm{m} / \mathrm{z} 246.1253$, found $\mathrm{m} / \mathrm{z} 246.1259$. The enantiomers were analyzed by HPLC using a chiral OD-H column (n-heptane/2-propanol $=99 / 1$, flow rate $=1.0 \mathrm{~mL} / \mathrm{min}$, wavelength $=254 \mathrm{~nm}$ ); minor enantiomer: $t_{R}=9.08 \mathrm{~min}$, major enantiomer: $t_{R}=10.42 \min ; 97 \%$ ee.

7r: Light yellow oil; Yield: $84 \%$, purification by flash chromatography (hexane/EtOAc $=$ 98/2); $[\boldsymbol{\alpha}]_{\mathrm{D}}{ }^{20}=-23.86\left(c=0.352\right.$, EtOAc); ${ }^{1} \mathrm{H}$ NMR $\left(600 \mathrm{MHz}, \mathrm{CDCl}_{3}\right): \boldsymbol{\delta}=0.88(\mathrm{t}, J=7.68$ $\mathrm{Hz}, 3 \mathrm{H}), 1.25-1.43(\mathrm{~m}, 4 \mathrm{H}), 1.73-1.83$ (m, 2H), 4.07 (brs, 1H), 4.28 (t, J=6.78 Hz, 1H), $6.50(\mathrm{~d}, J=7.74 \mathrm{~Hz}, 2 \mathrm{H}), 6.61(\mathrm{t}, J=7.32 \mathrm{~Hz}, 1 \mathrm{H}), 7.07(\mathrm{t}, J=7.14 \mathrm{~Hz}, 2 \mathrm{H}), 7.21(\mathrm{t}, J=$ $6.90 \mathrm{~Hz}, 1 \mathrm{H}), 7.29-7.34(\mathrm{~m}, 4 \mathrm{H}) ;{ }^{13} \mathrm{C} \mathrm{NMR}\left(150 \mathrm{MHz}, \mathrm{CDCl}_{3}\right): \boldsymbol{\delta}=14.0,22.6,28.5,38.8$, 58.2, 113.2, 117.1, 126.4, 126.9, 128.5, 129.1, 144.4, 147.5; ESI HRMS exact mass calcd. for $\mathrm{C}_{17} \mathrm{H}_{22} \mathrm{~N}$ requires $\mathrm{m} / \mathrm{z} 240.1747$, found $\mathrm{m} / \mathrm{z} 240.1749$.

The enantiomers were analyzed by HPLC using a chiral OD-H column (n-heptane/2-propanol $=99 / 1$, flow rate $=1.0 \mathrm{~mL} / \mathrm{min}$, wavelength $=254 \mathrm{~nm}$ ); minor enantiomer: $t_{R}=6.73 \mathrm{~min}$, major enantiomer: $t_{R}=8.23 \mathrm{~min} ; 89 \%$ ee. 
7s: Light yellow oil; Yield: $86 \%$, purification by flash chromatography (hexane/EtOAc $=$ 98/2); $[\boldsymbol{\alpha}]_{\mathrm{D}}{ }^{20}=-18.78(c=0.362, \mathrm{EtOAc}) ;{ }^{1} \mathrm{H}$ NMR $\left(300 \mathrm{MHz}, \mathrm{CDCl}_{3}\right): \boldsymbol{\delta}=0.94(\mathrm{~d}, J=6.23$ Hz, 3H), 0.99 (d, J = 6.09 Hz, 3H), $1.57-1.62$ (m, 1H), $1.65-1.74$ (m, 2H), 4.04 (brs, 1H), $4.38(\mathrm{t}, J=7.54 \mathrm{~Hz}, 1 \mathrm{H}), 6.52(\mathrm{~d}, J=7.60 \mathrm{~Hz}, 2 \mathrm{H}), 6.63(\mathrm{t}, J=7.32 \mathrm{~Hz}, 1 \mathrm{H}), 7.06-7.11(\mathrm{~m}$, 2H), $7.22-7.37(\mathrm{~m}, 5 \mathrm{H}) ;{ }^{13} \mathrm{C}$ NMR $\left(150 \mathrm{MHz}, \mathrm{CDCl}_{3}\right): \boldsymbol{\delta}=22.4,23.0,25.1$, 48.6, 56.2, 113.2, 117.1, 126.3, 126.9, 128.6, 129.1, 144.8, 147.5; ESI HRMS exact mass calcd. for $\mathrm{C}_{17} \mathrm{H}_{22} \mathrm{~N}$ requires $\mathrm{m} / \mathrm{z} 240.1747$, found $\mathrm{m} / \mathrm{z} 240.1740$.

The enantiomers were analyzed by HPLC using a chiral OD-H column (n-heptane/2-propanol $=99 / 1$, flow rate $=1.0 \mathrm{~mL} / \mathrm{min}$, wavelength $=254 \mathrm{~nm}$ ); minor enantiomer: $t_{\mathrm{R}}=6.68 \mathrm{~min}$, major enantiomer: $t_{R}=7.55 \mathrm{~min} ; 91 \%$ ee. 
The NMR spectra of $\mathbf{4 a}$

Bruker Avance 600 probe: 13C-1H DUL sample: CMN7-18B solveat: CDCL3 spectrum: 1H

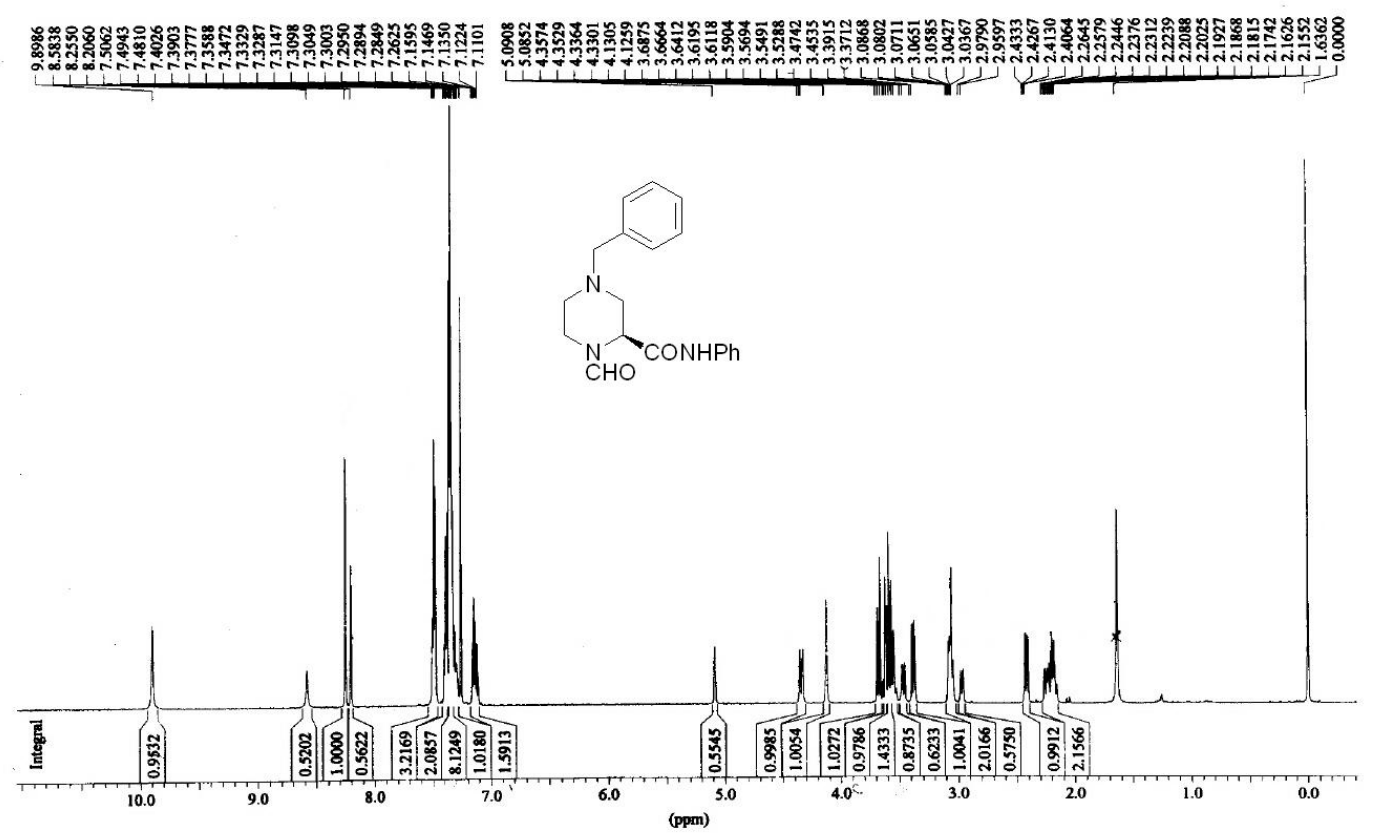

Bruker Avanse 600 probe: $13 \mathrm{C}-1 \mathrm{H}$ DUL sample: CMN7-18B solvent: $\mathrm{CDCL} 3$ spectrum: $13 \mathrm{C}$

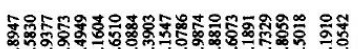

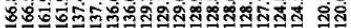

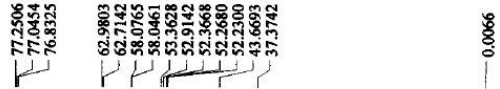
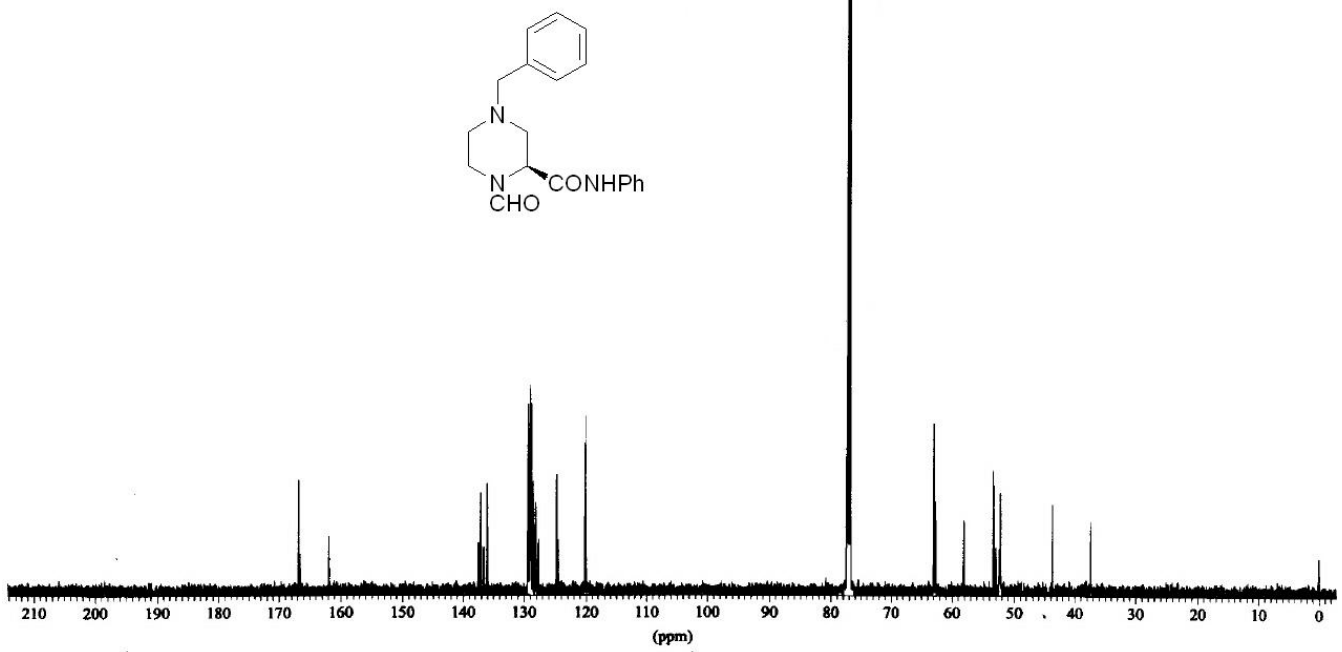
The NMR spectra of $\mathbf{4 b}$

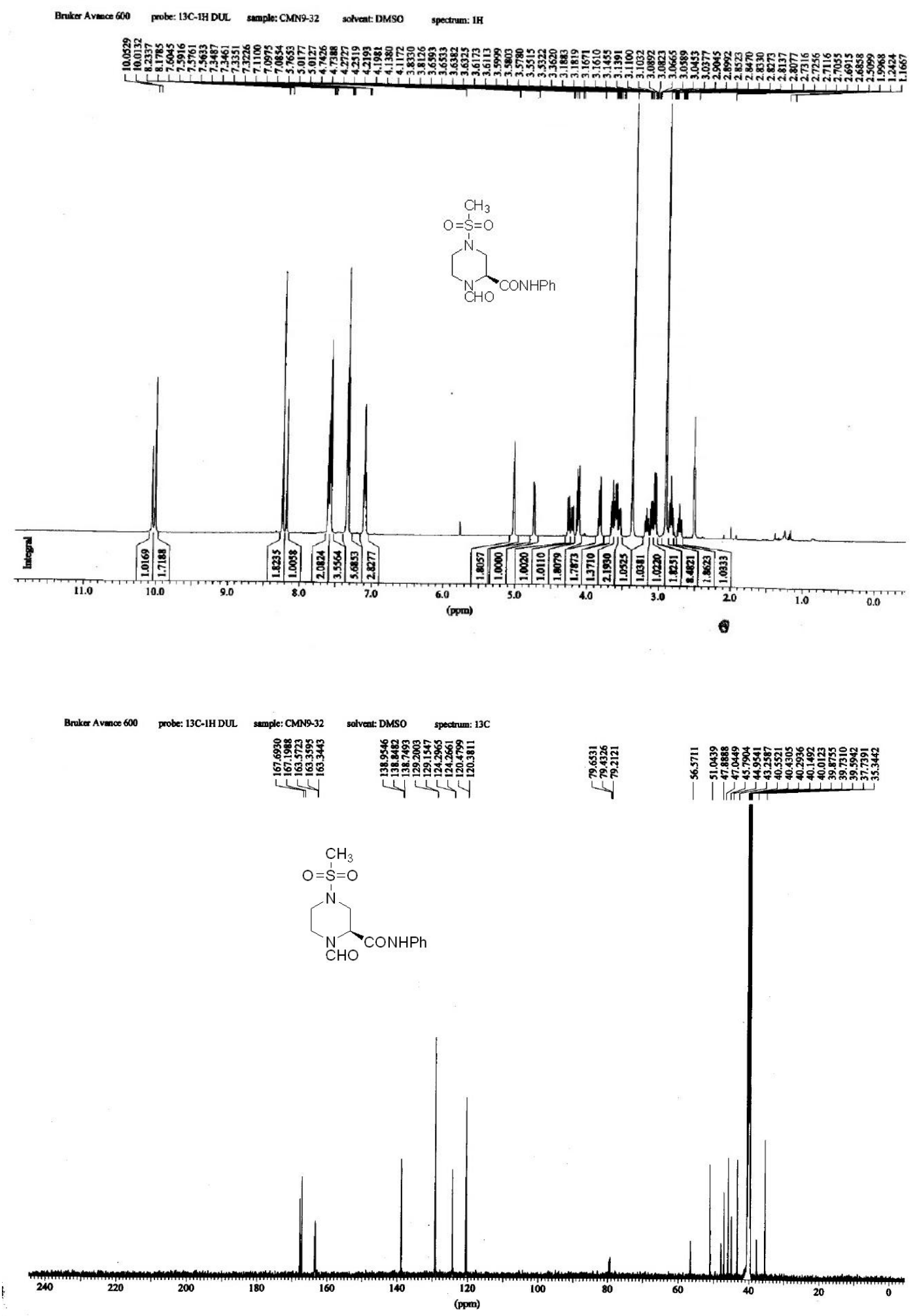


The NMR spectra of $\mathbf{4 c}$

Bruker Avance 600 probe: 13C-1H DUL sample: CMN7-24B
solvent: CDCL3
MeOD spectrum: 1H

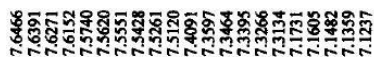 \\ IIIT)}
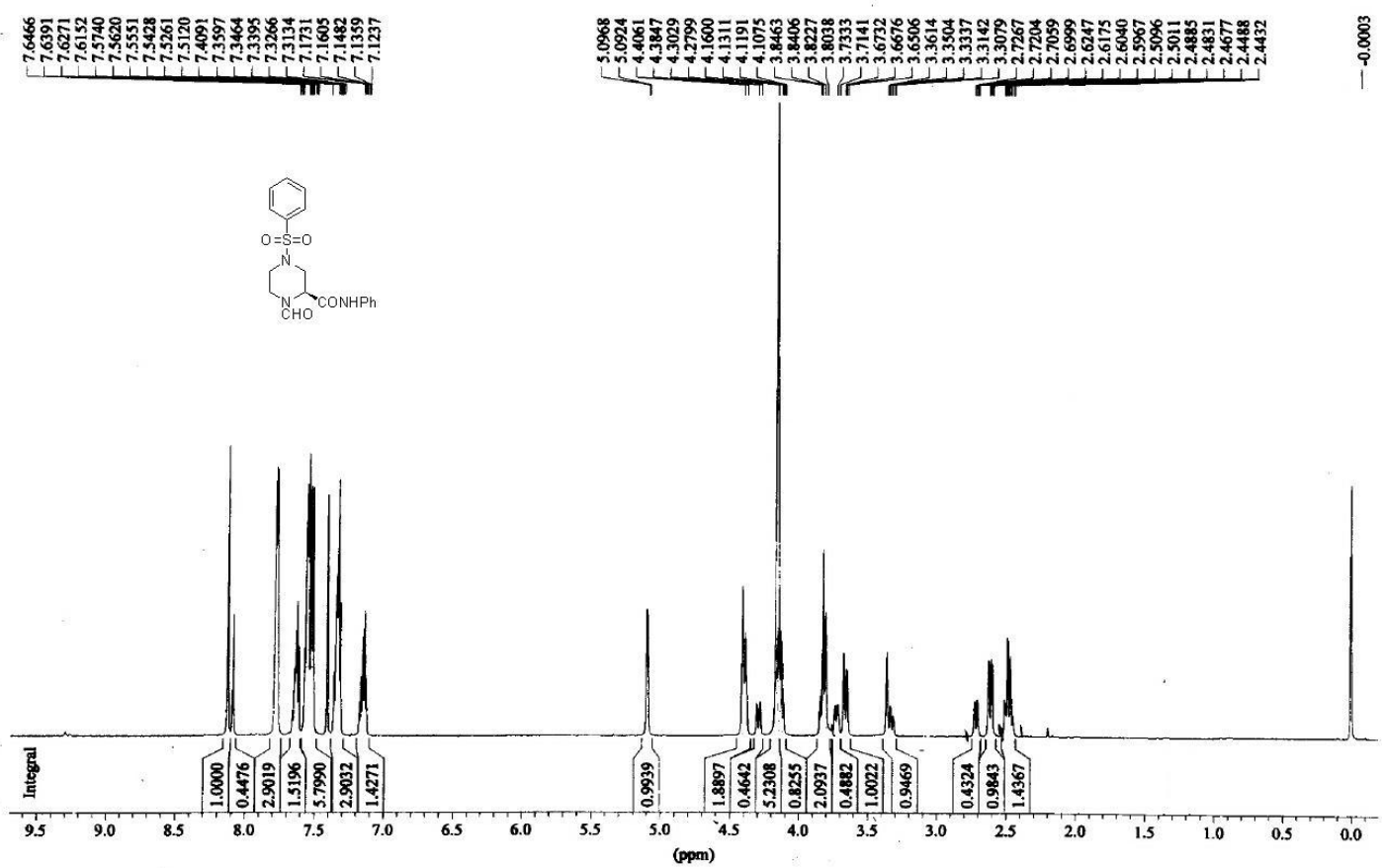

Bruker Avance 600 probe: $13 C-1 \mathrm{H}$ DUL sample: CMN7-24B solvent: CDCL3
MeOD spectrum: $13 \mathrm{C}$

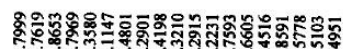

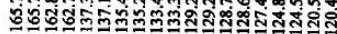

$4 y$ 11

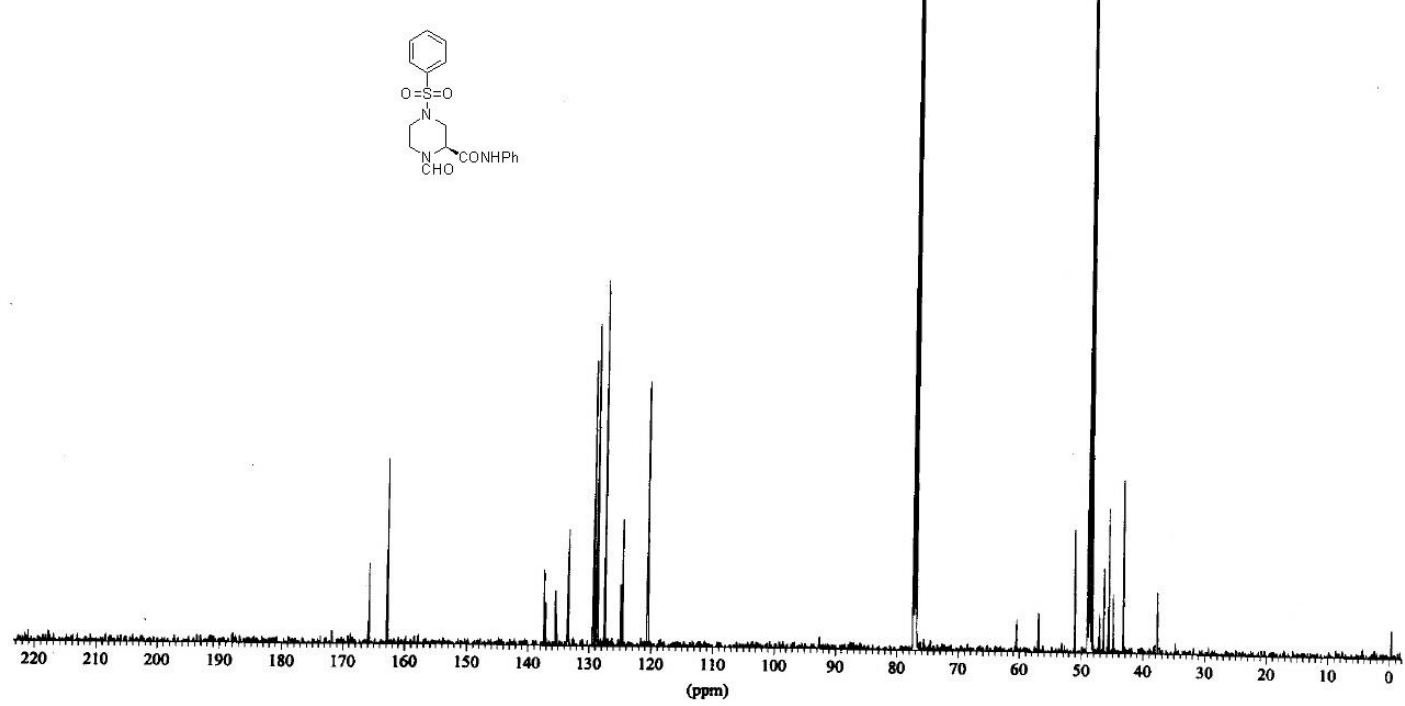


The NMR spectra of $\mathbf{4 d}$
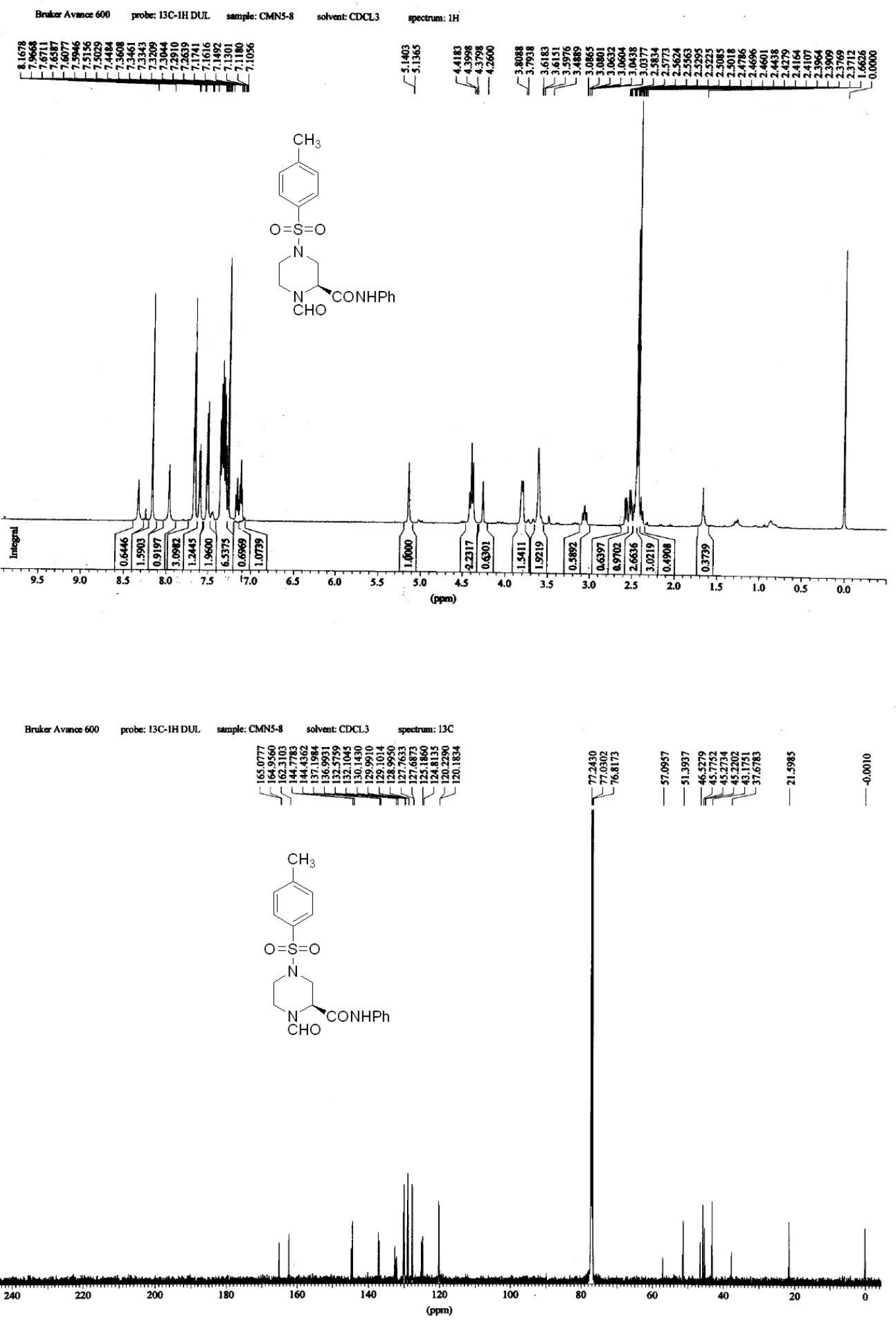
The NMR spectra of $\mathbf{4 e}$

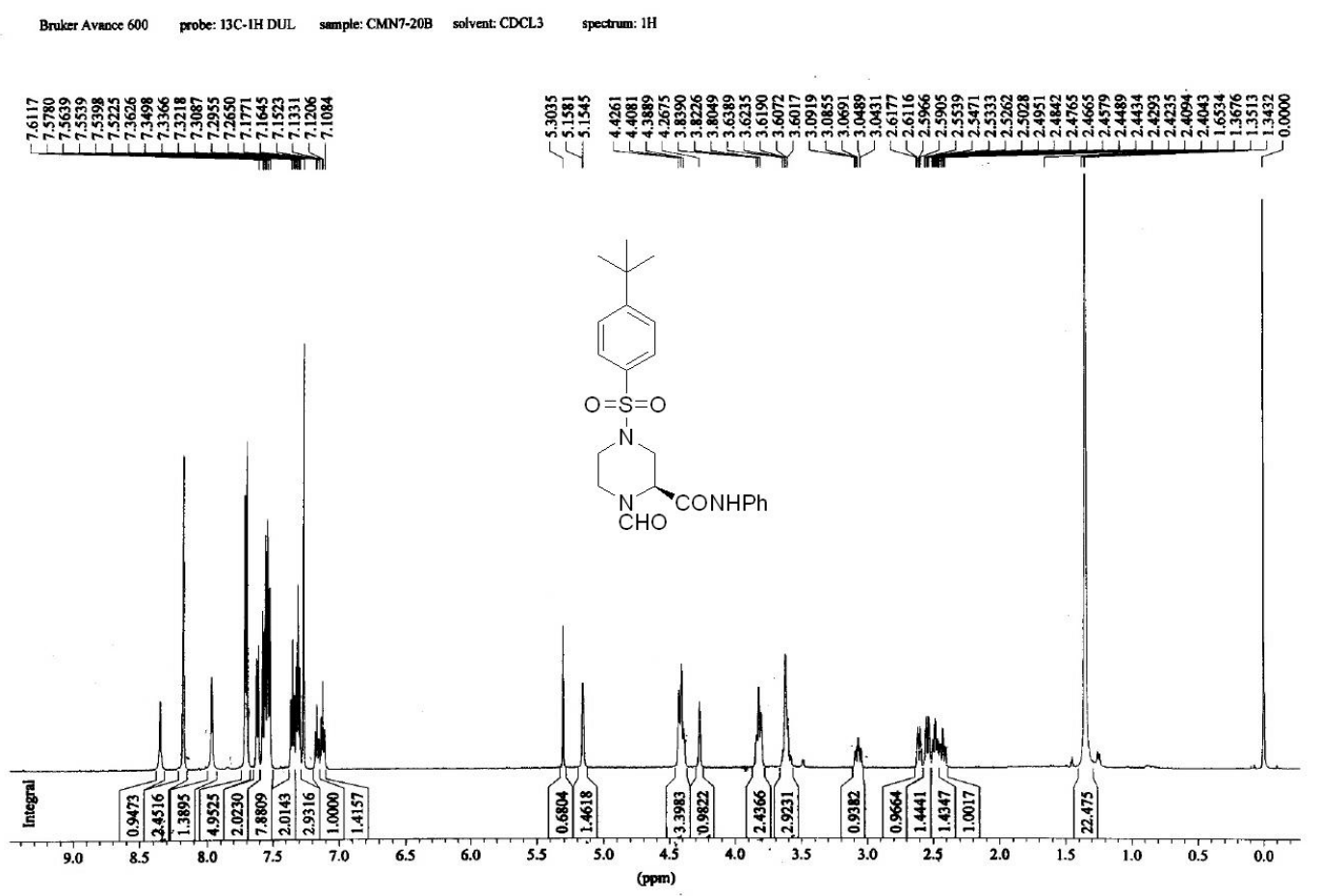

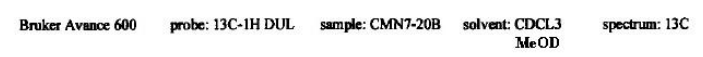

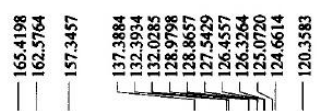

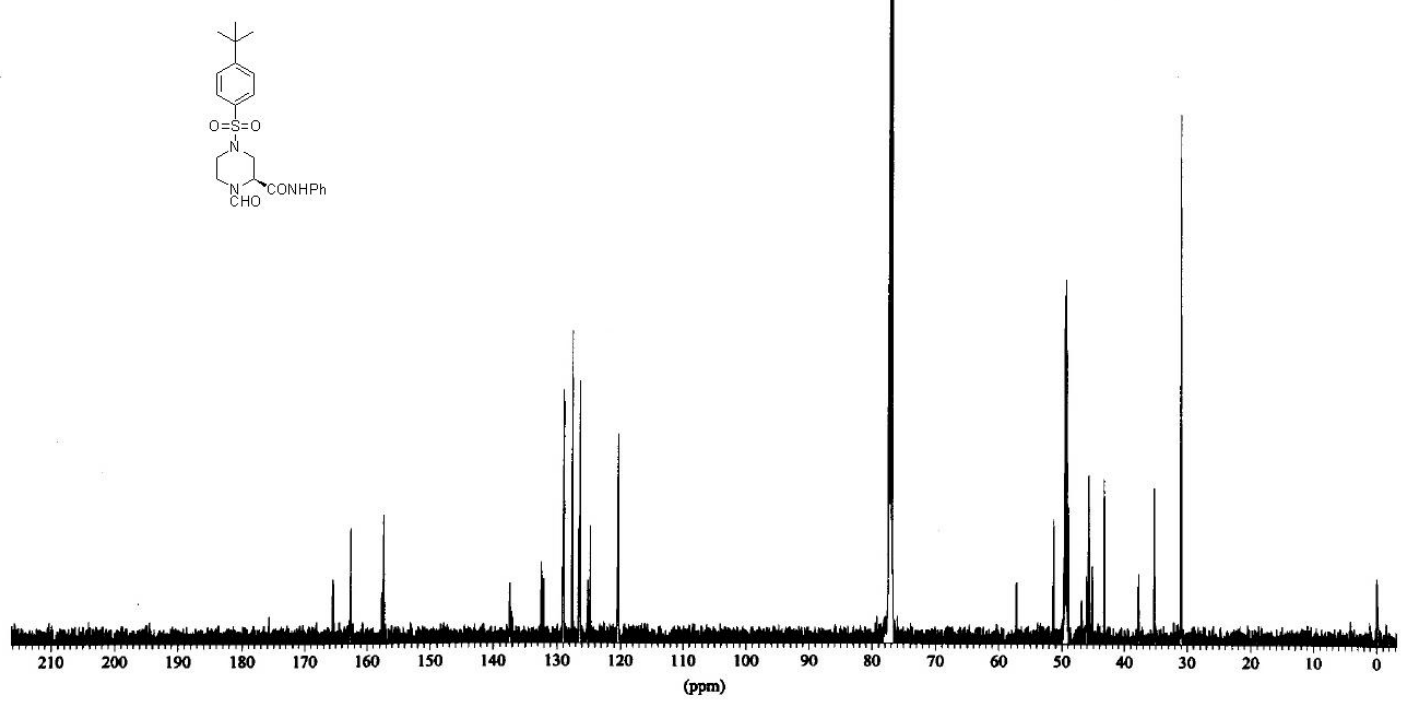

$210 \quad 200$

$\begin{array}{lllllll}190 & 180 & 170 & 160 & 150\end{array}$
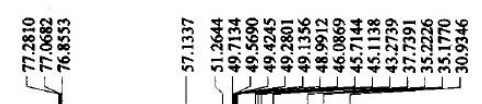

i্ 
The NMR spectra of $\mathbf{5 a}$
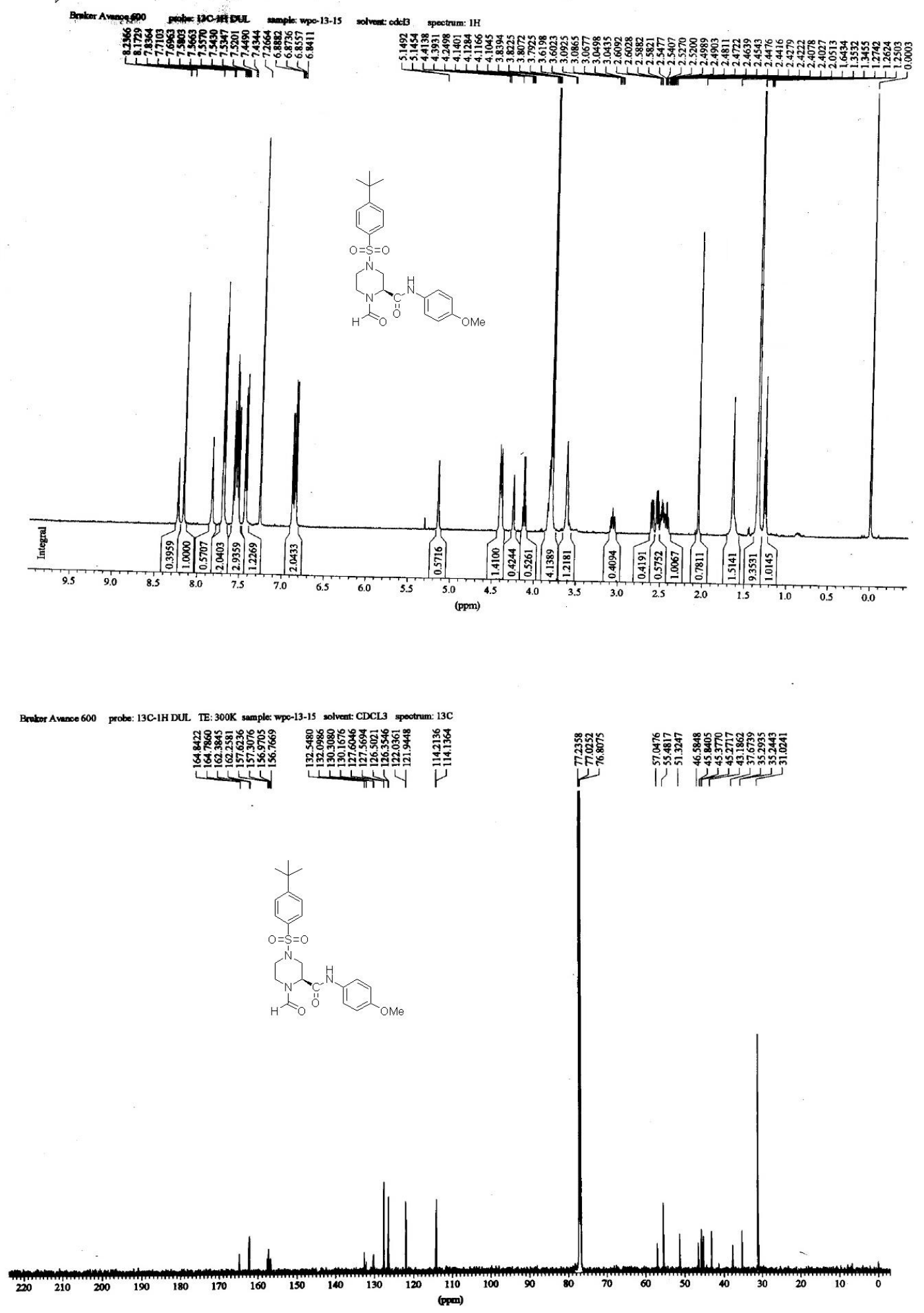
The NMR spectra of $\mathbf{5 b}$
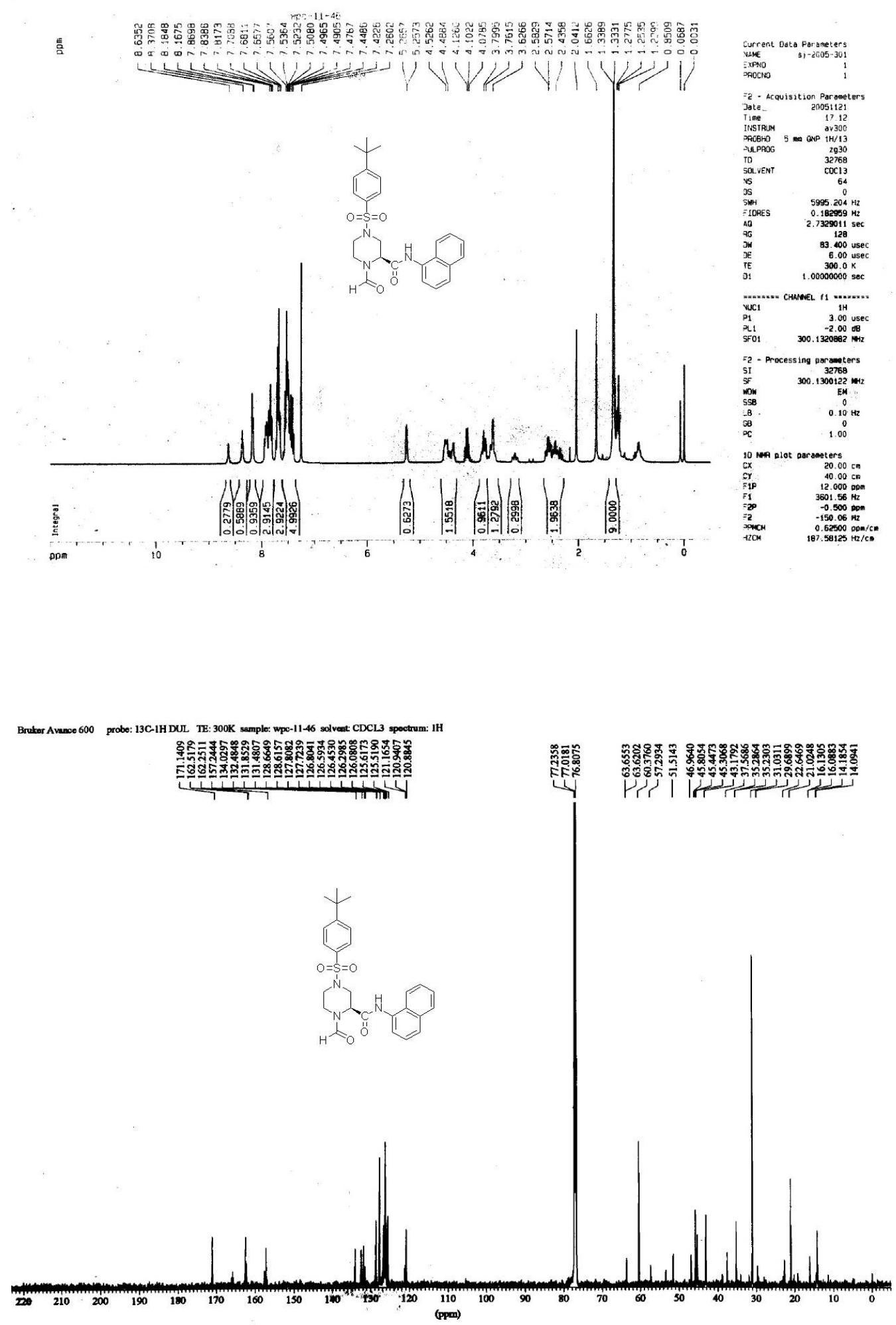
The NMR spectra of $\mathbf{5 c}$
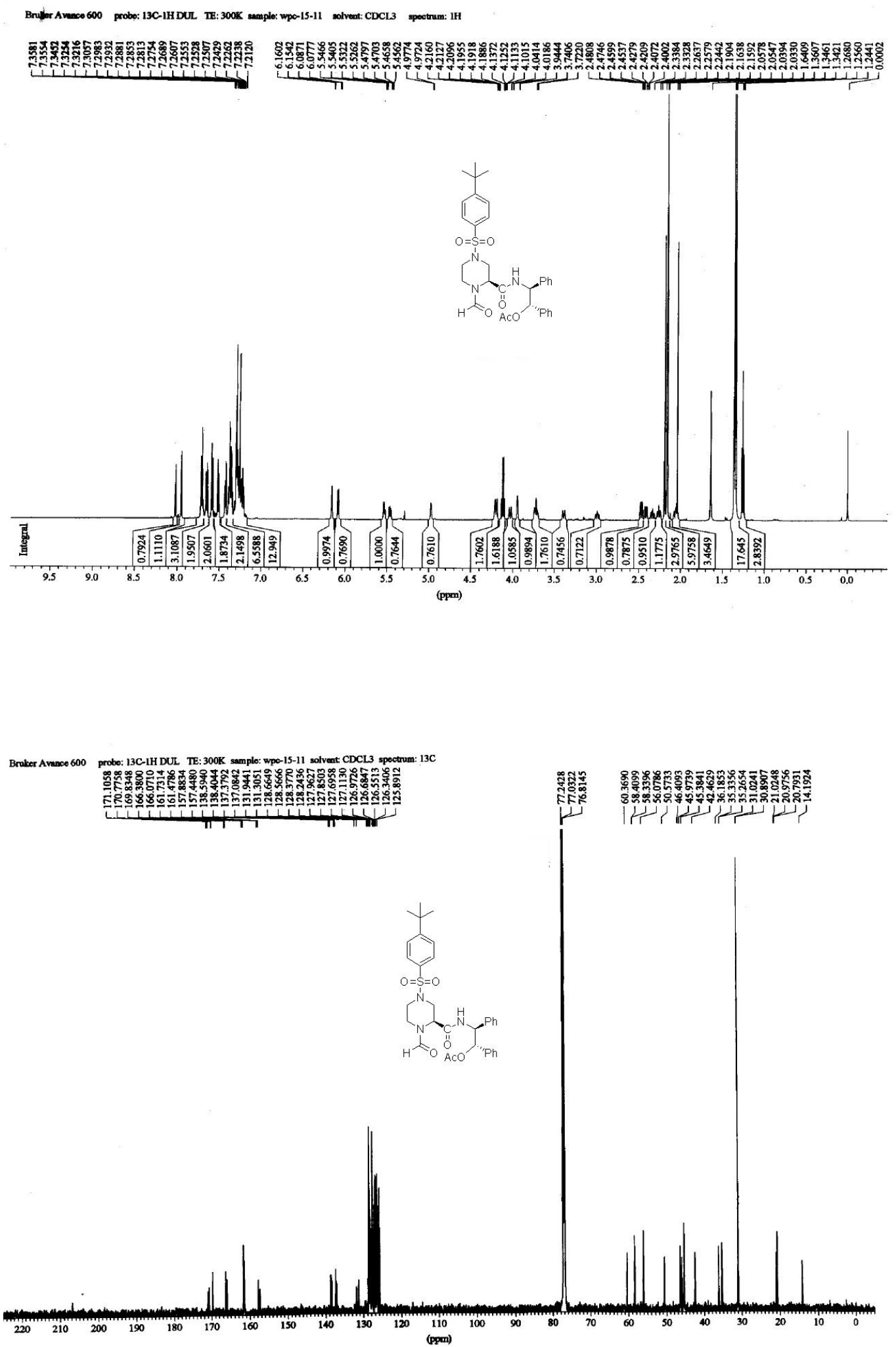
The NMR spectra of $\mathbf{5 d}$
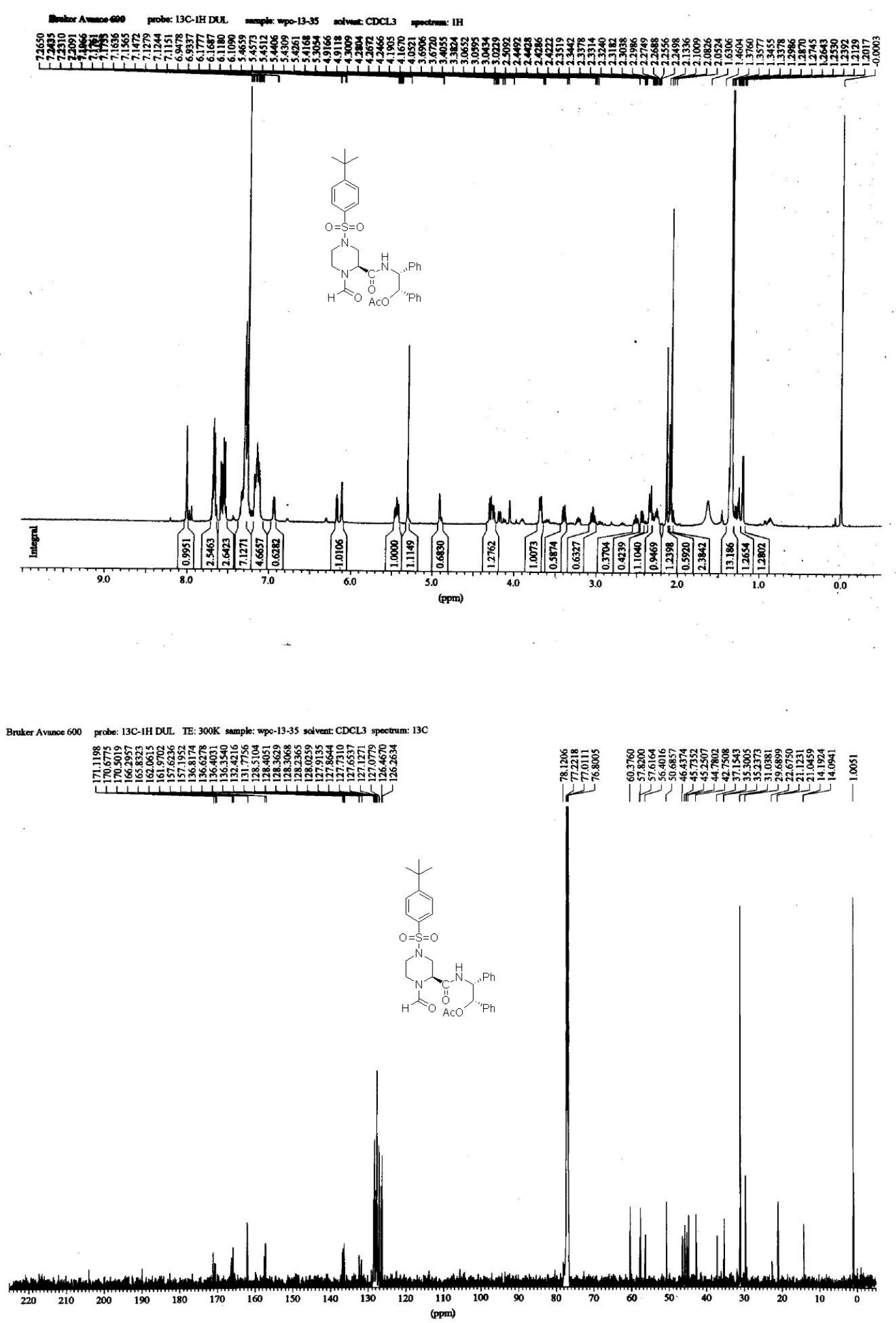
The NMR spectra of $\mathbf{5 e}$
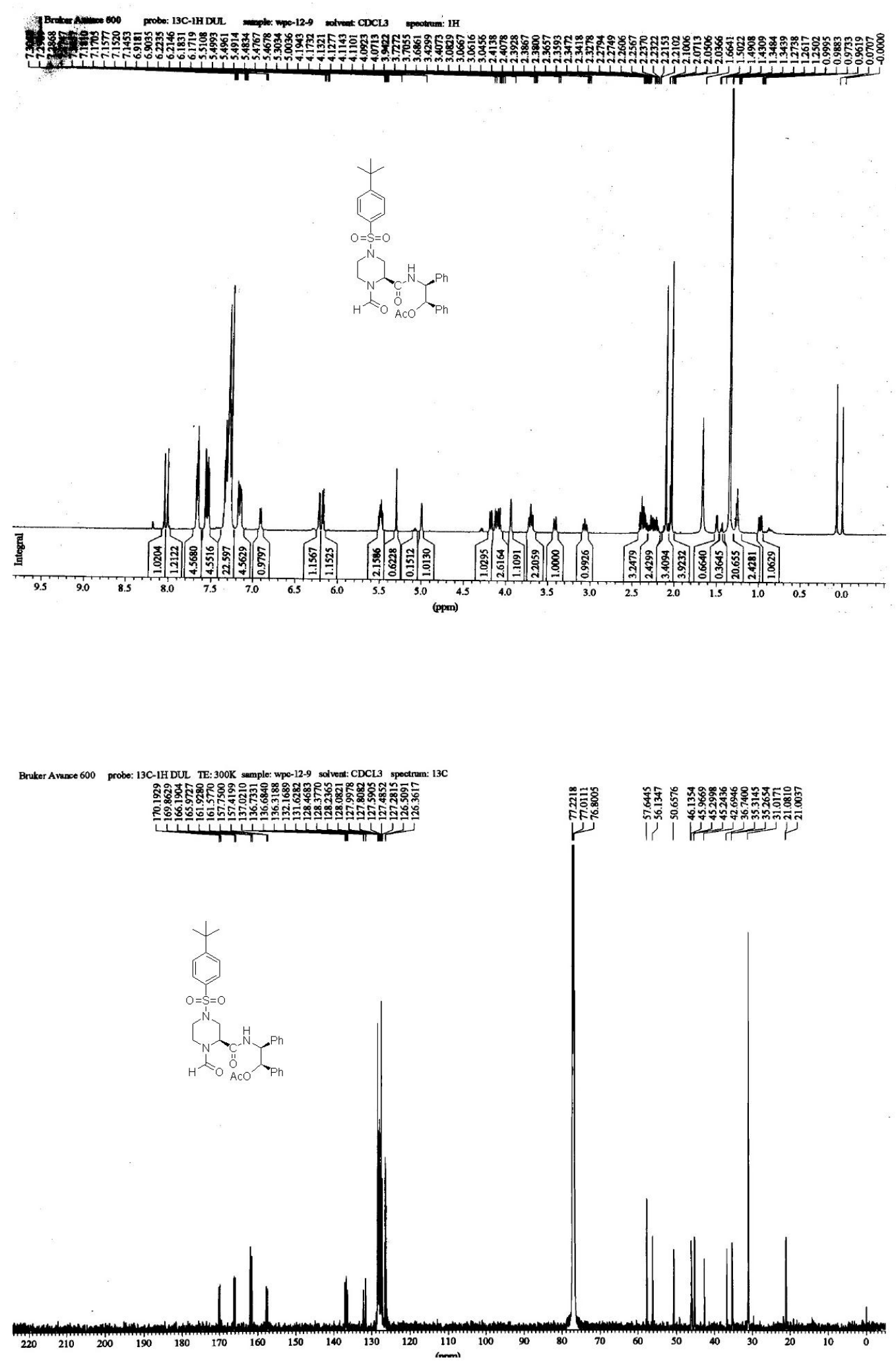
The NMR spectra of $\mathbf{6 h}$
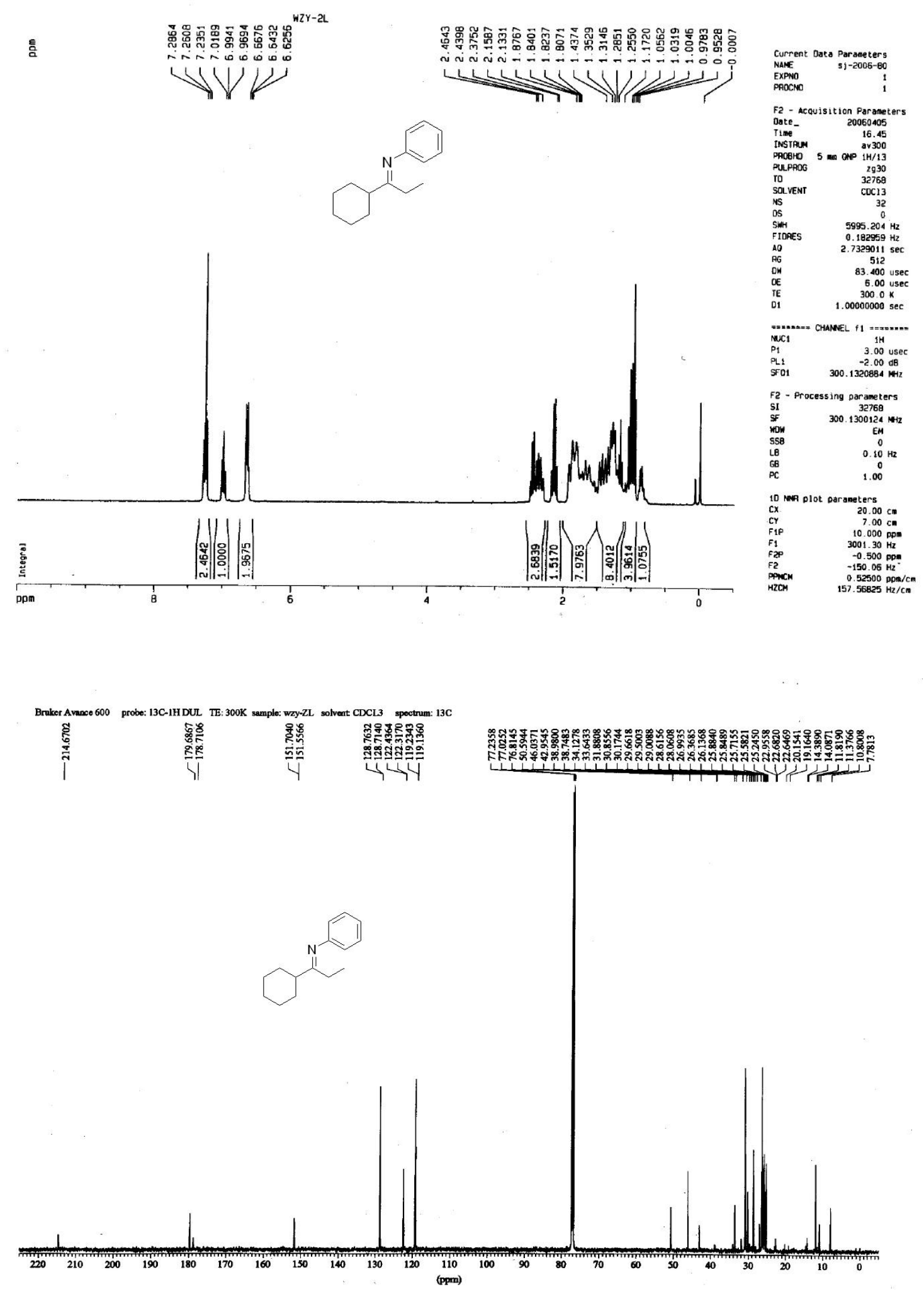
The NMR spectra of $\mathbf{6} \mathbf{j}$

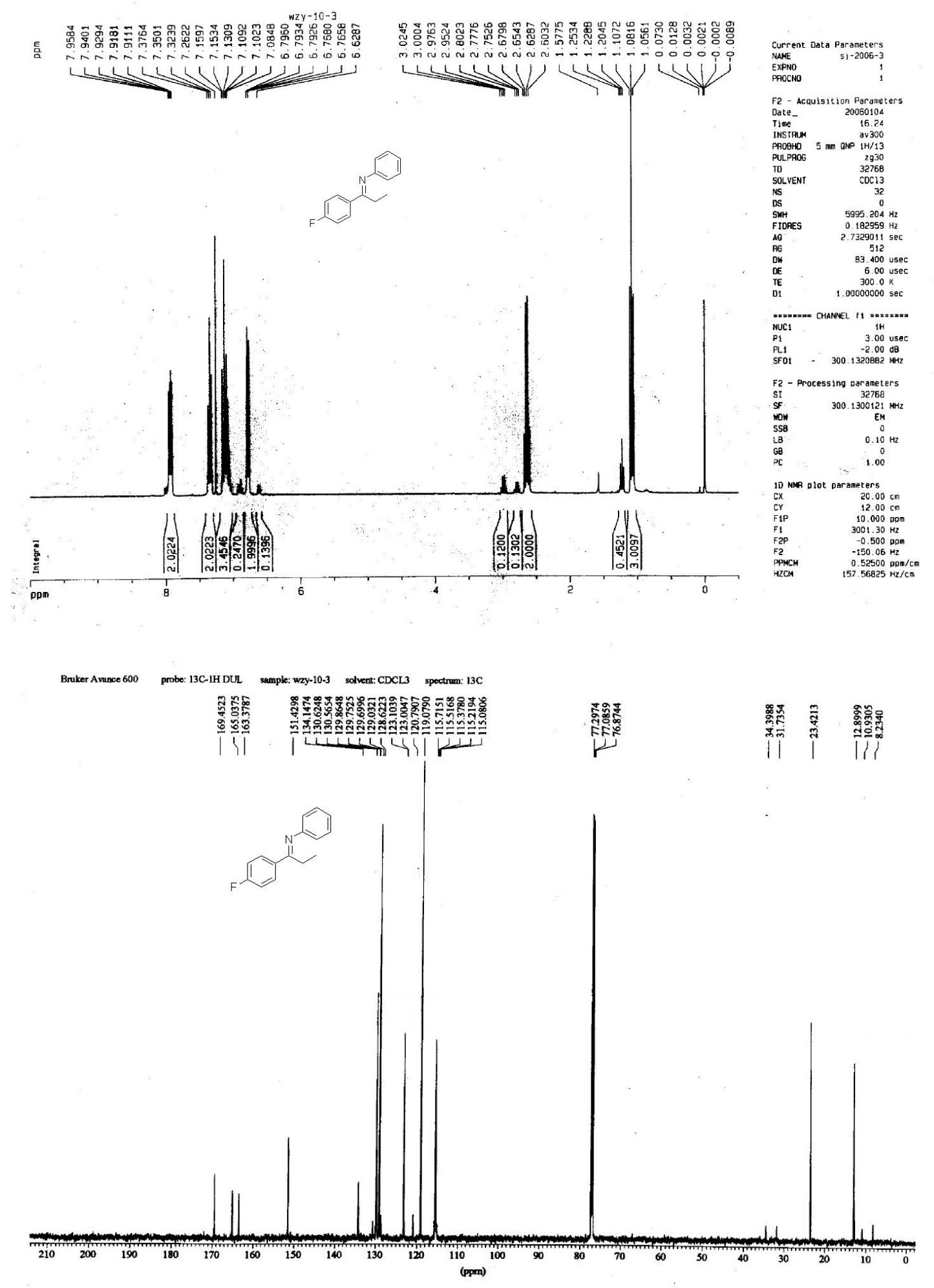


The NMR spectra of $\mathbf{6 k}$

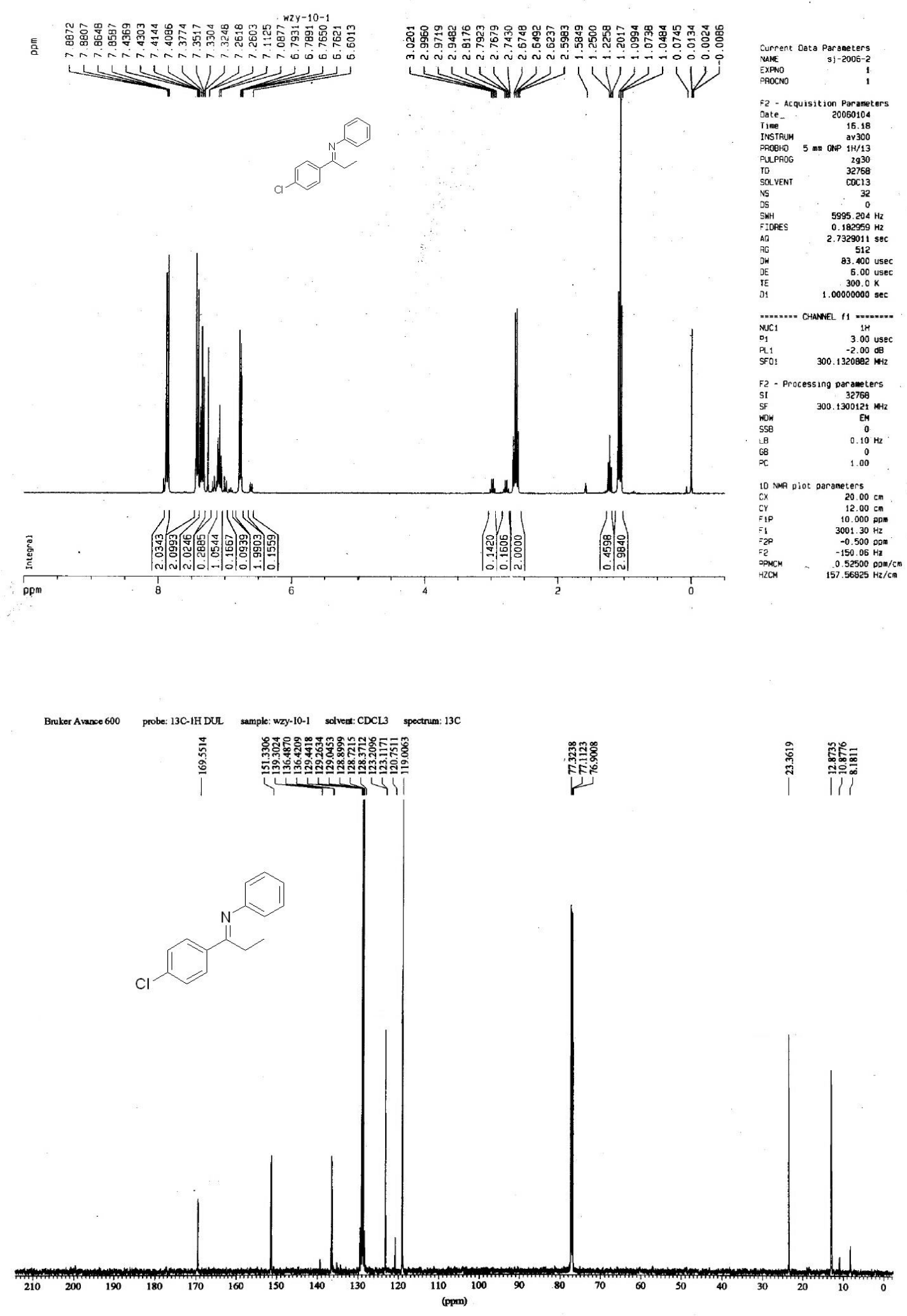


The NMR spectra of $\mathbf{6} \mathbf{1}$

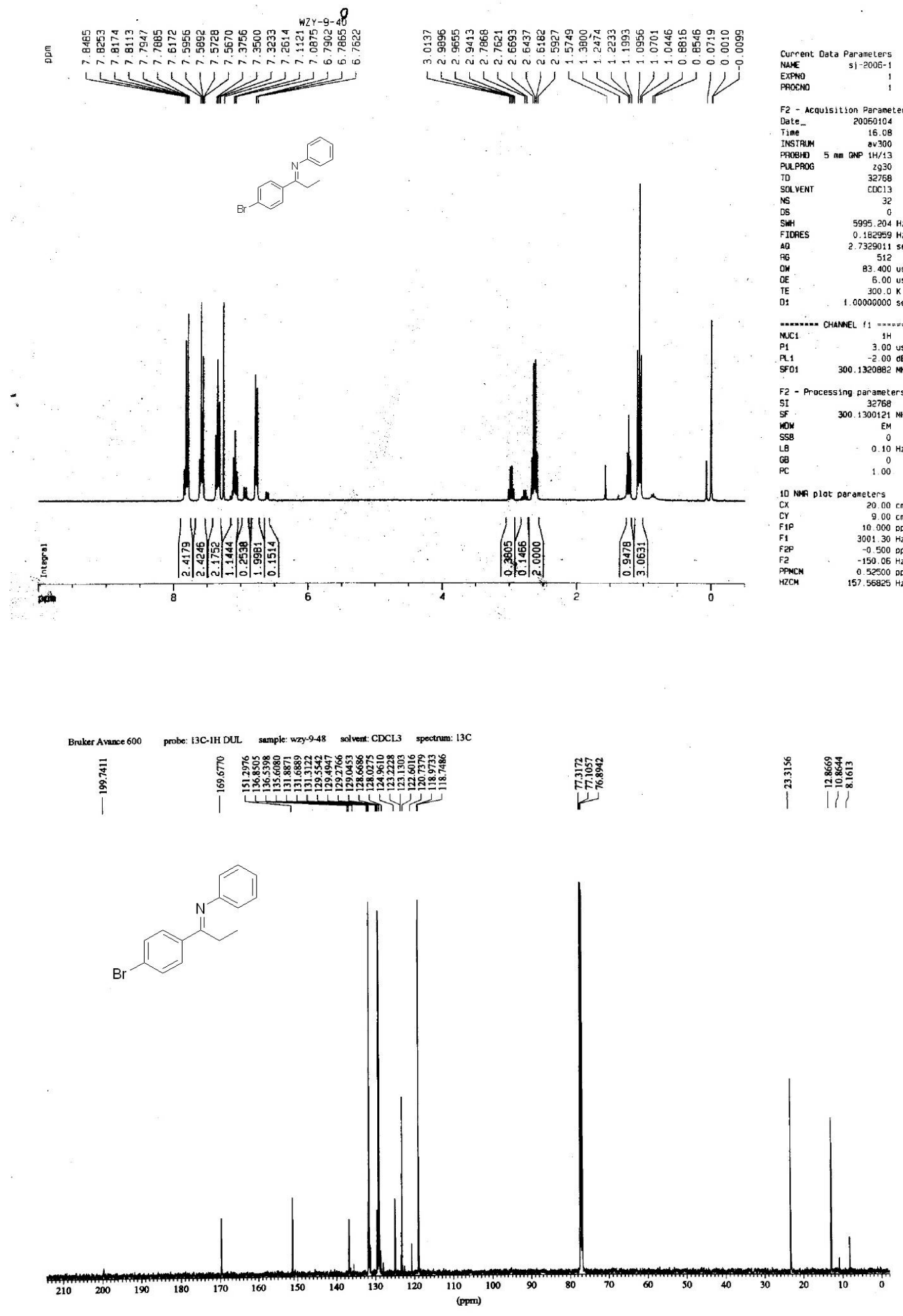


The NMR spectra of $\mathbf{6 m}$
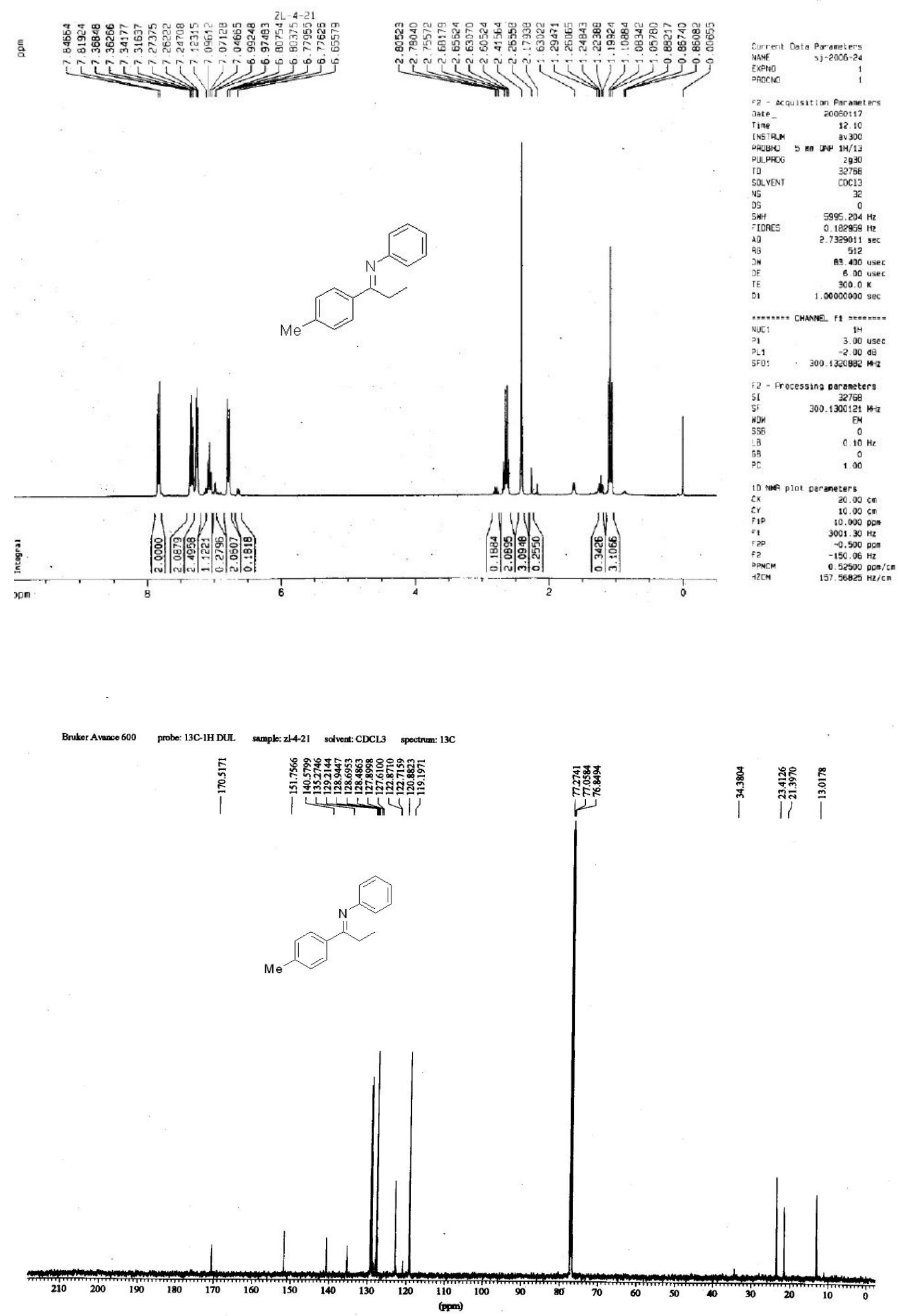
The NMR spectra of $\mathbf{6 n}$
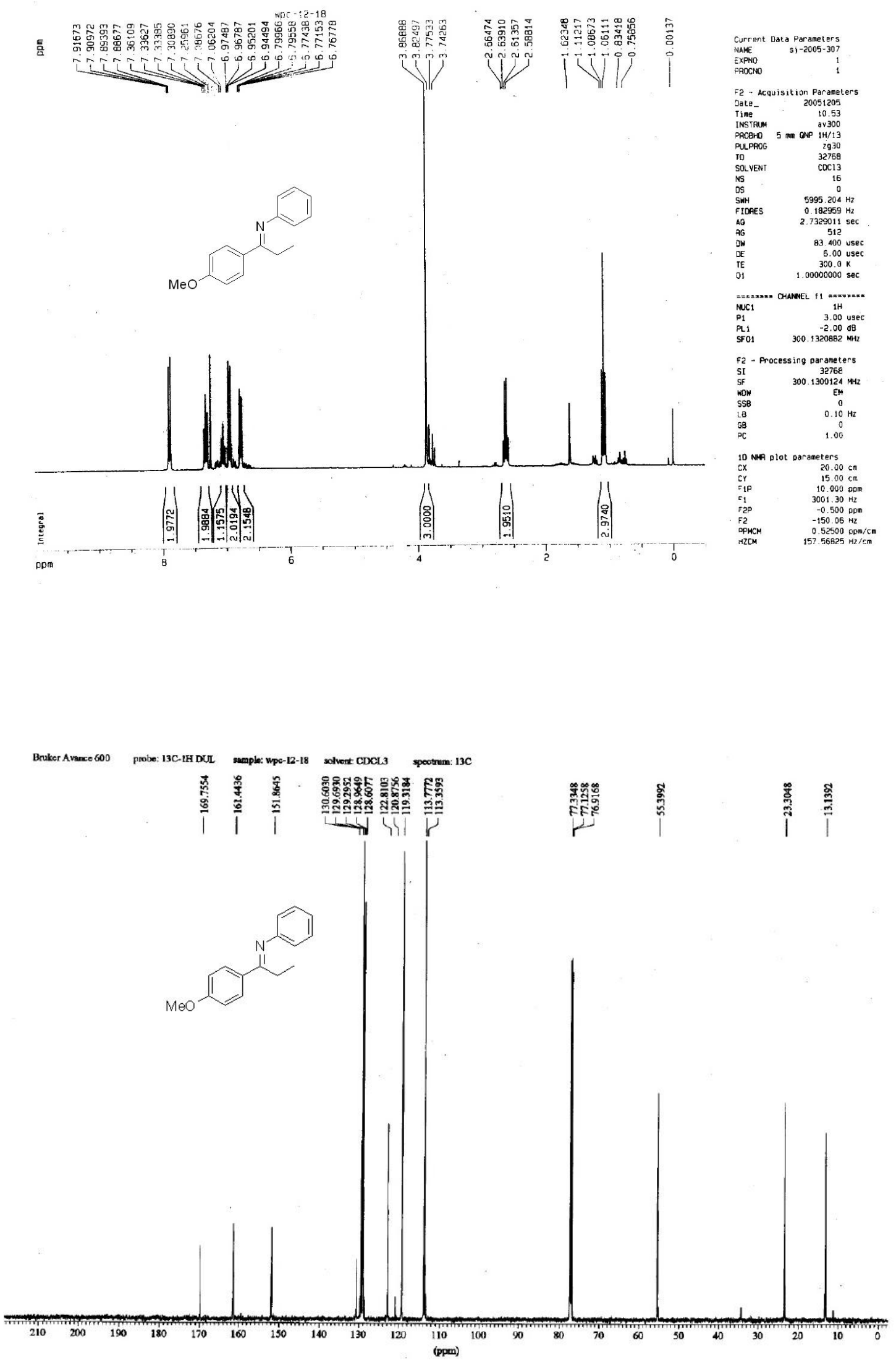
The NMR spectra of $\mathbf{6 q}$
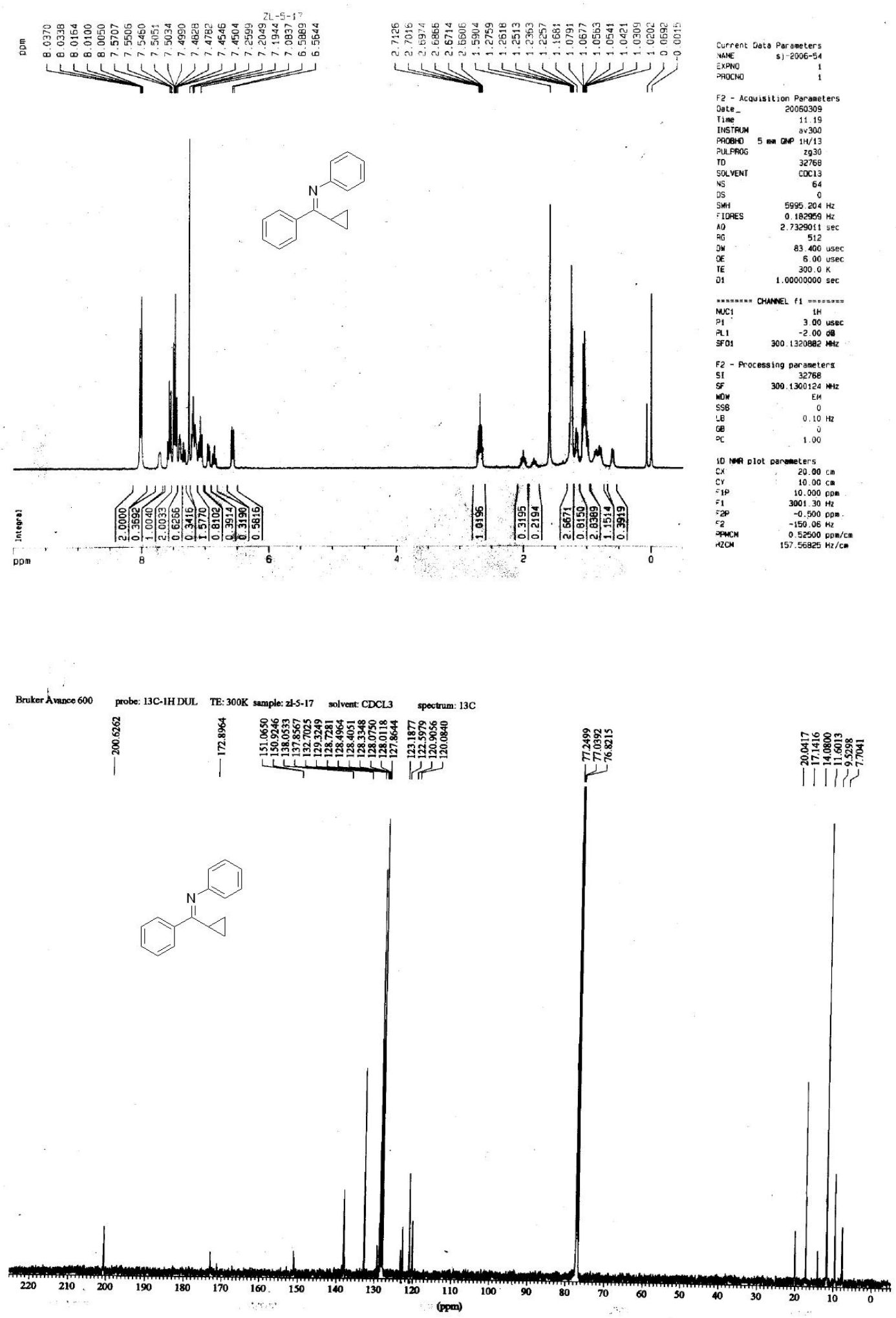
The NMR spectra of $\mathbf{6 r}$
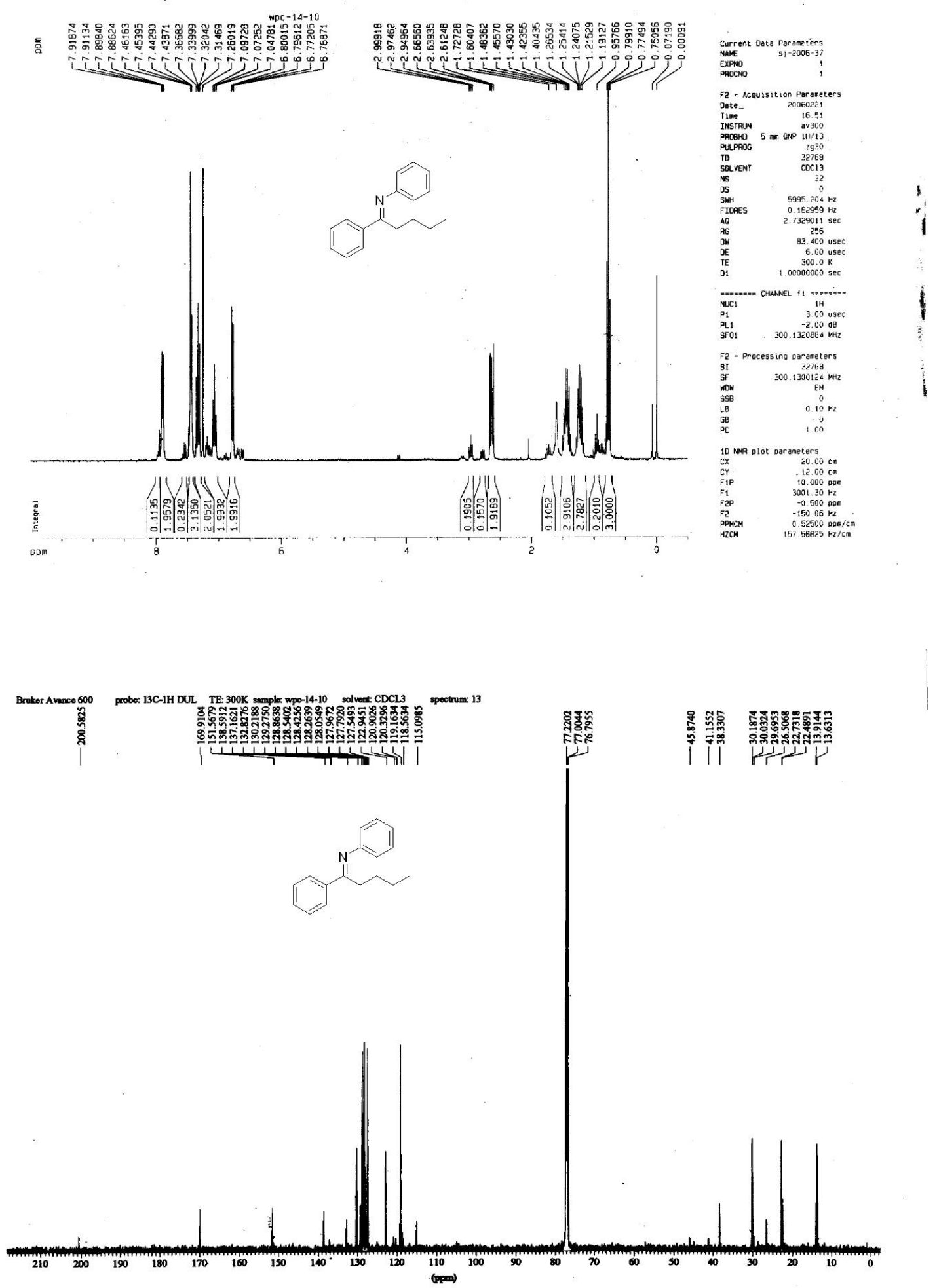
The NMR spectra of $\mathbf{6 s}$
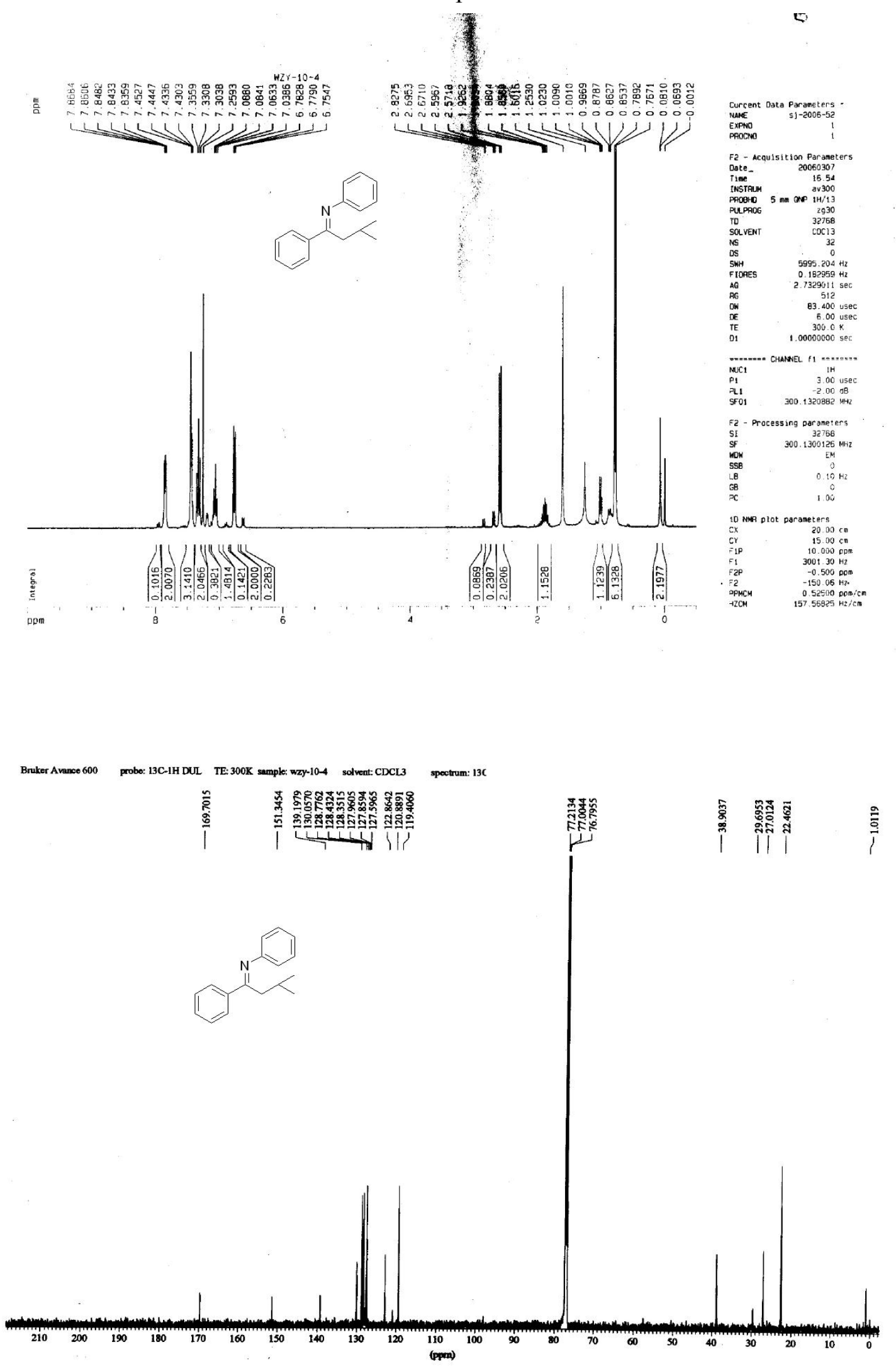
The NMR spectra of $\mathbf{7 h}$

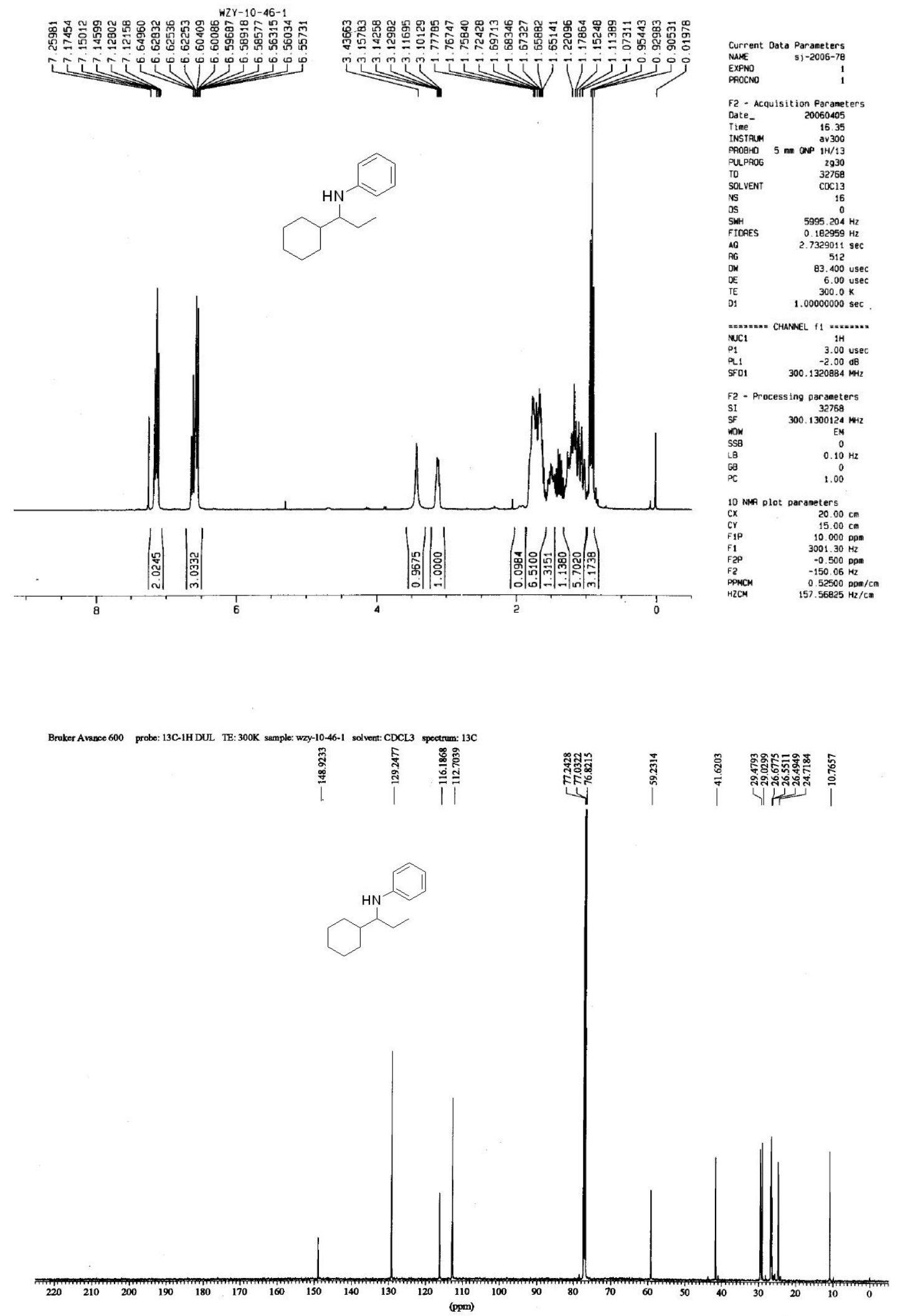


The NMR spectra of $N$-formyl-7h

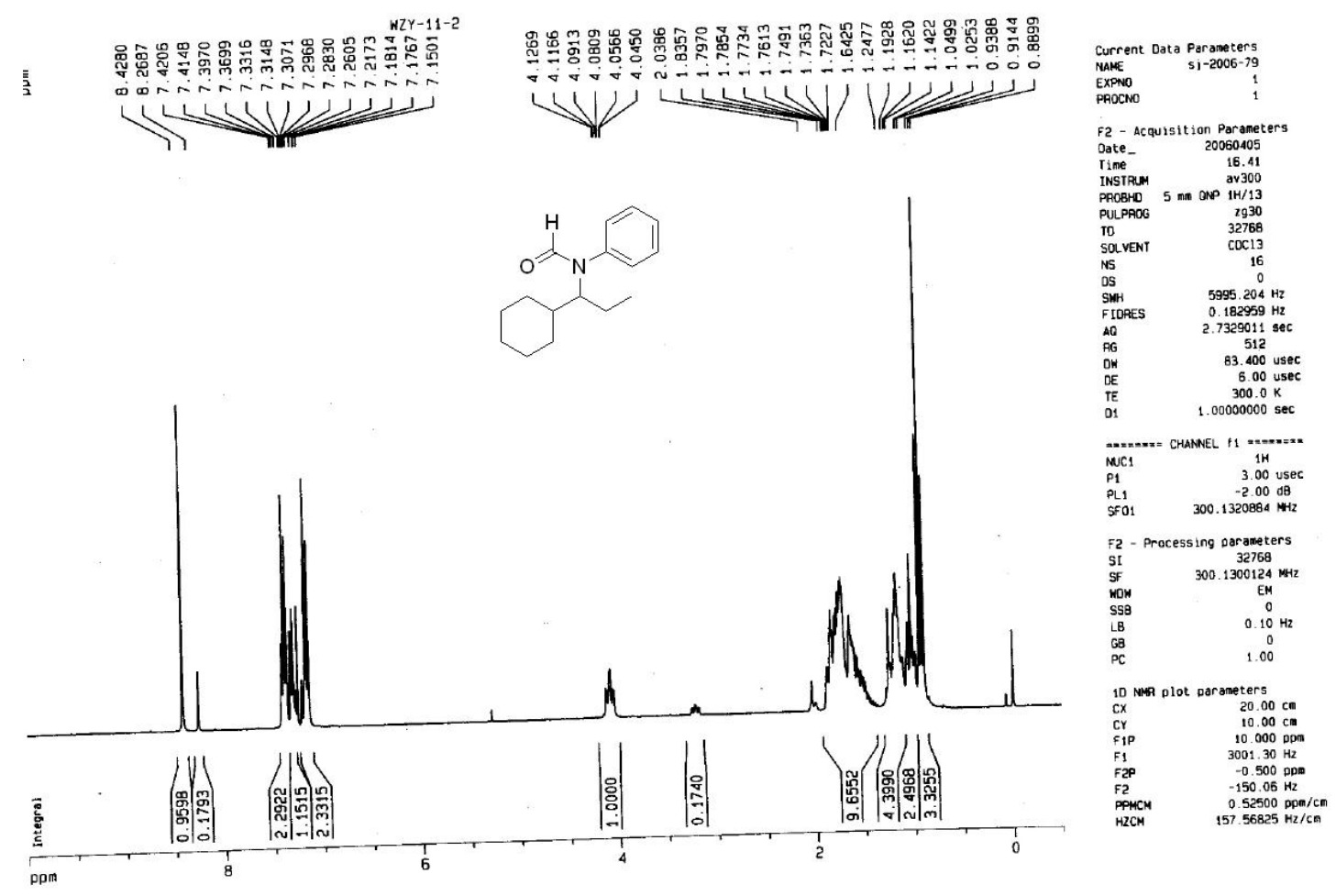


The NMR spectra of $\mathbf{7 j}$

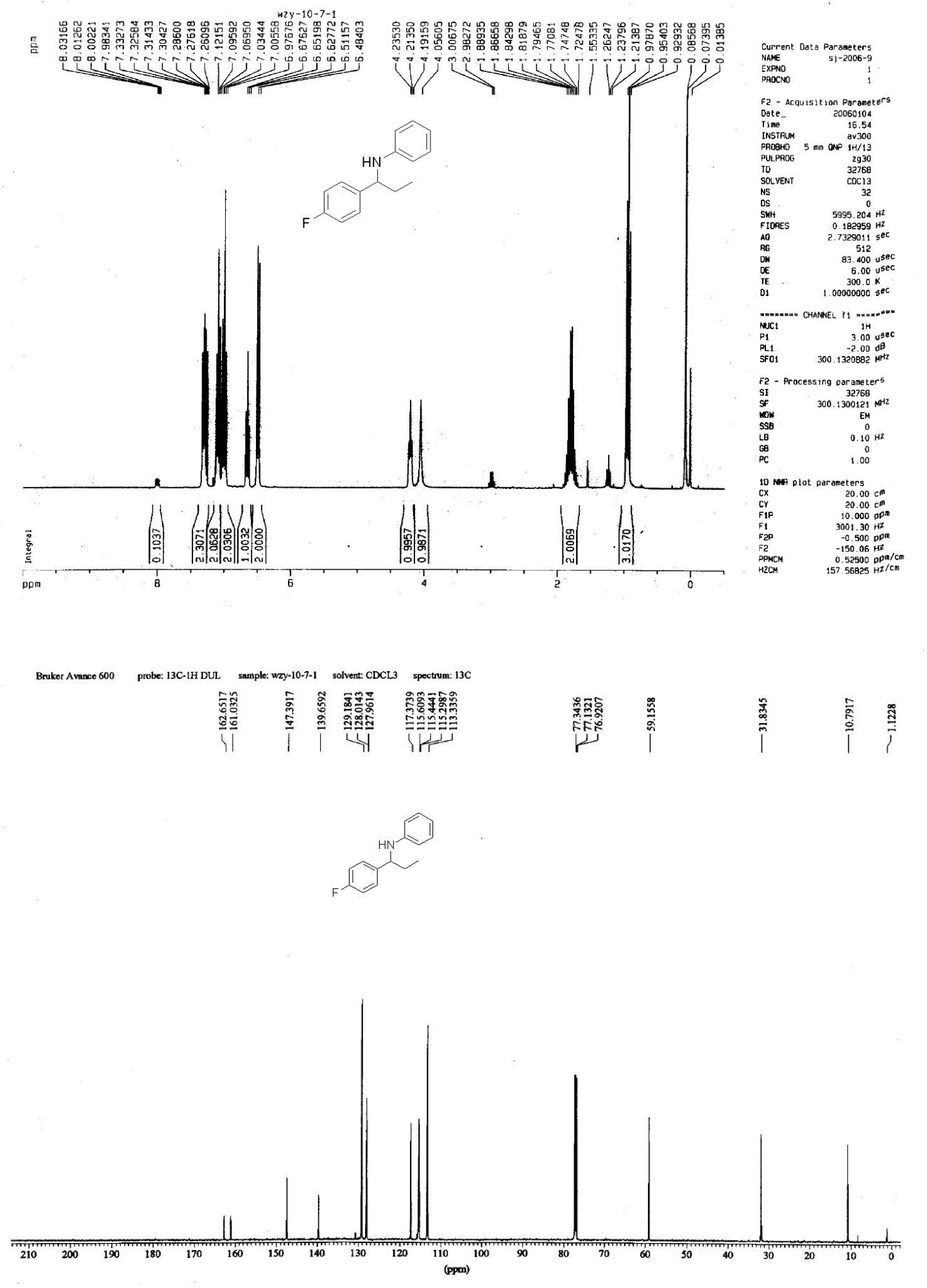


The NMR spectra of $\mathbf{7 k}$
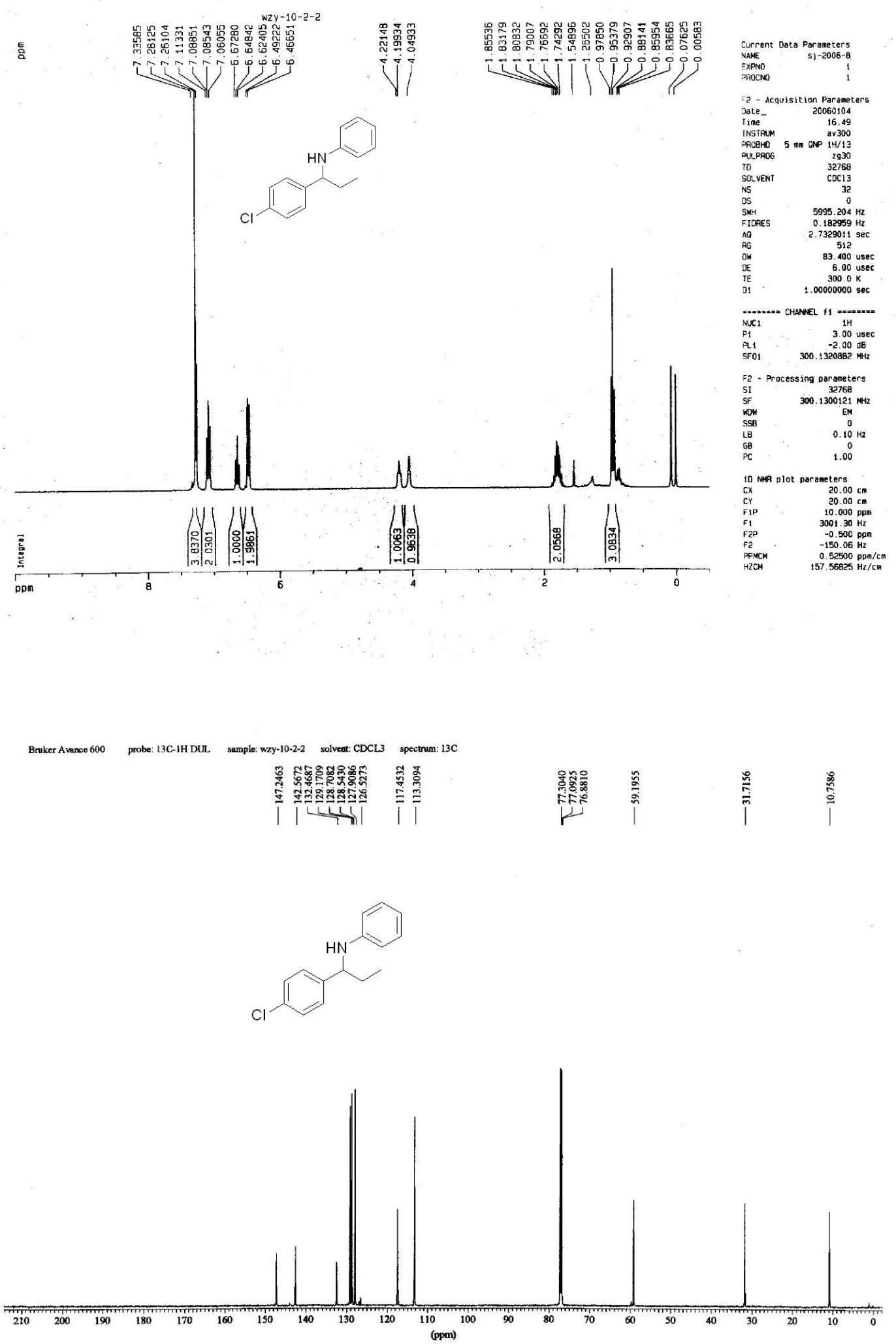
The NMR spectra of $7 \mathbf{l}$
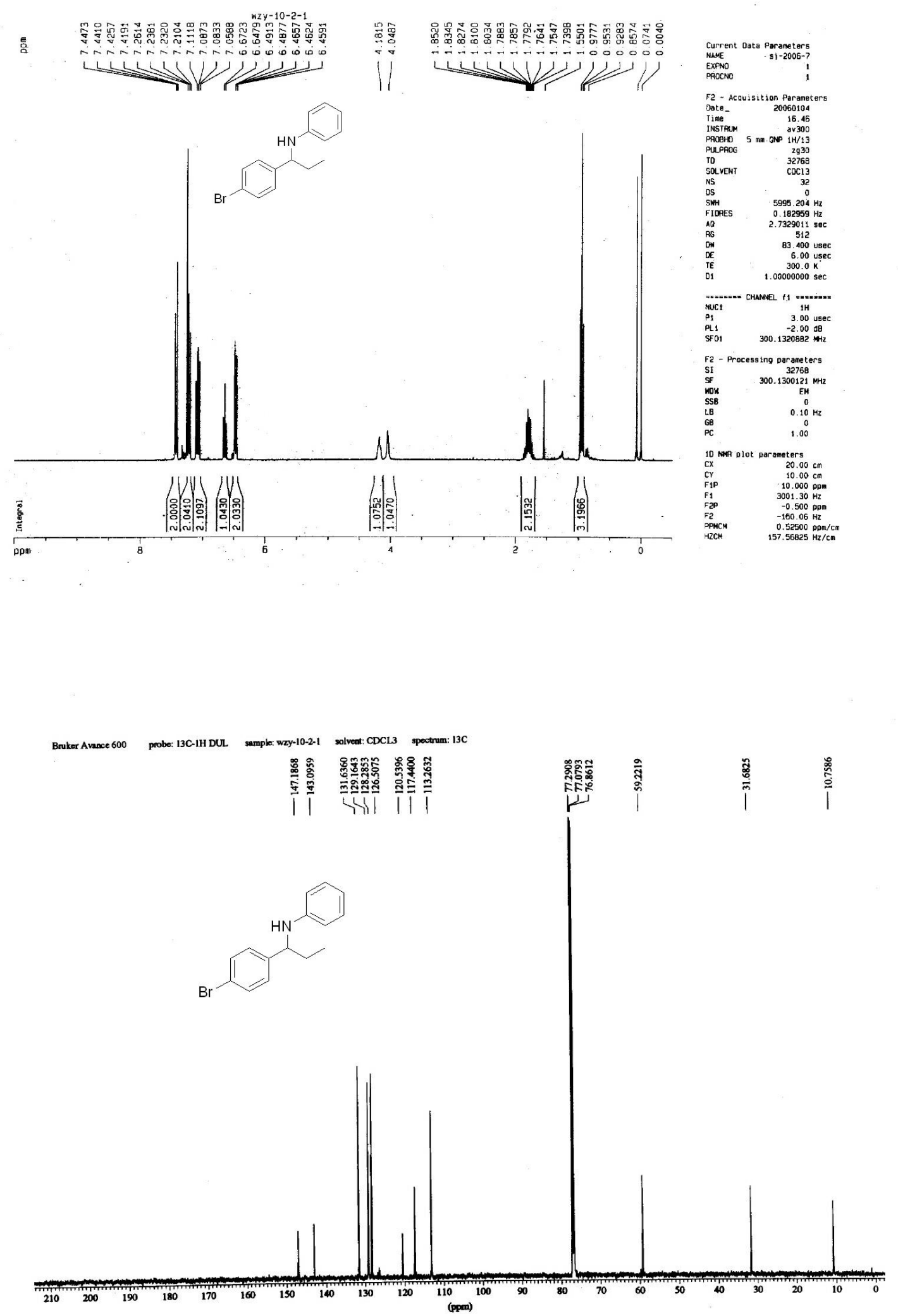
The NMR spectra of $\mathbf{7 m}$
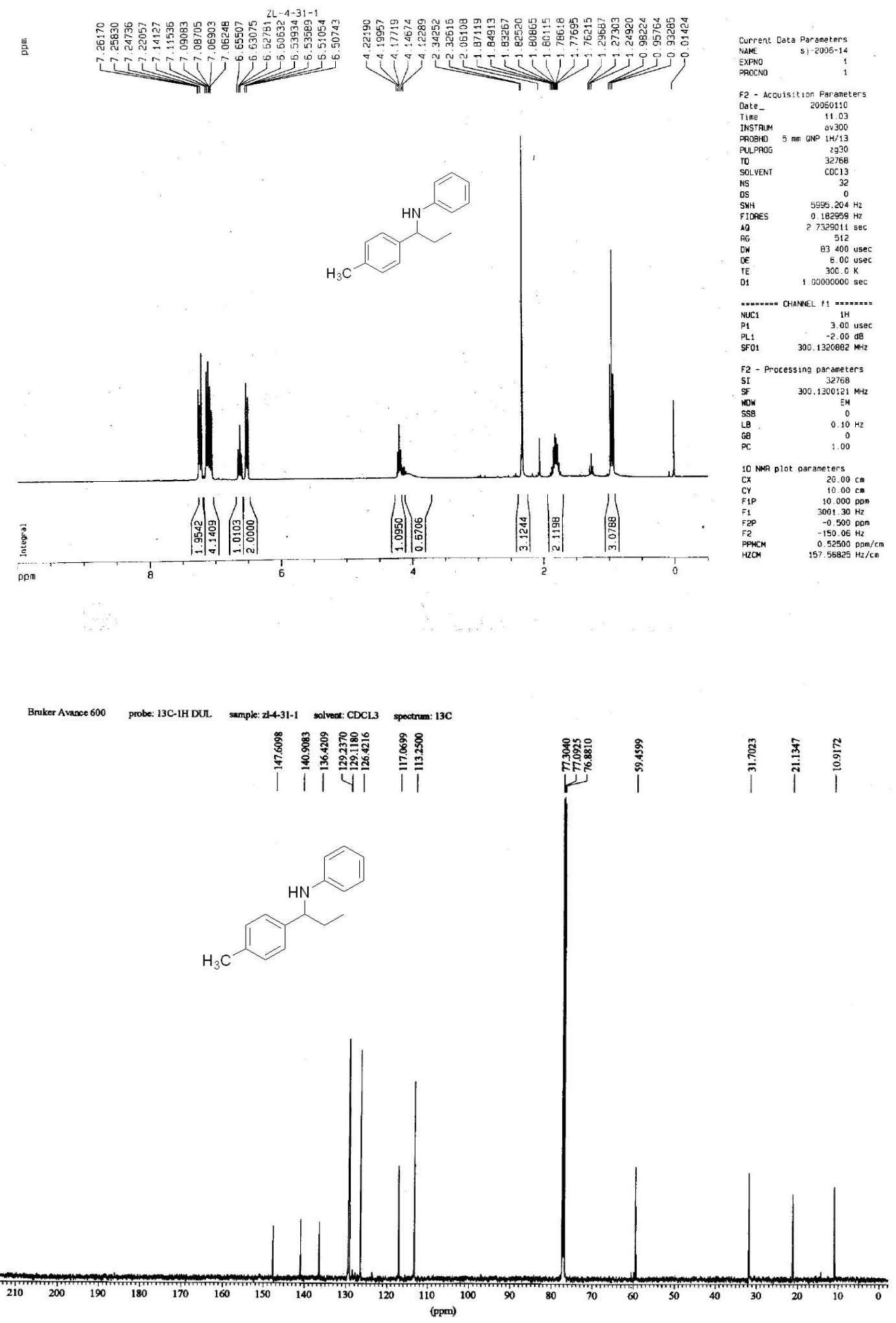
The NMR spectra of $\mathbf{7 n}$
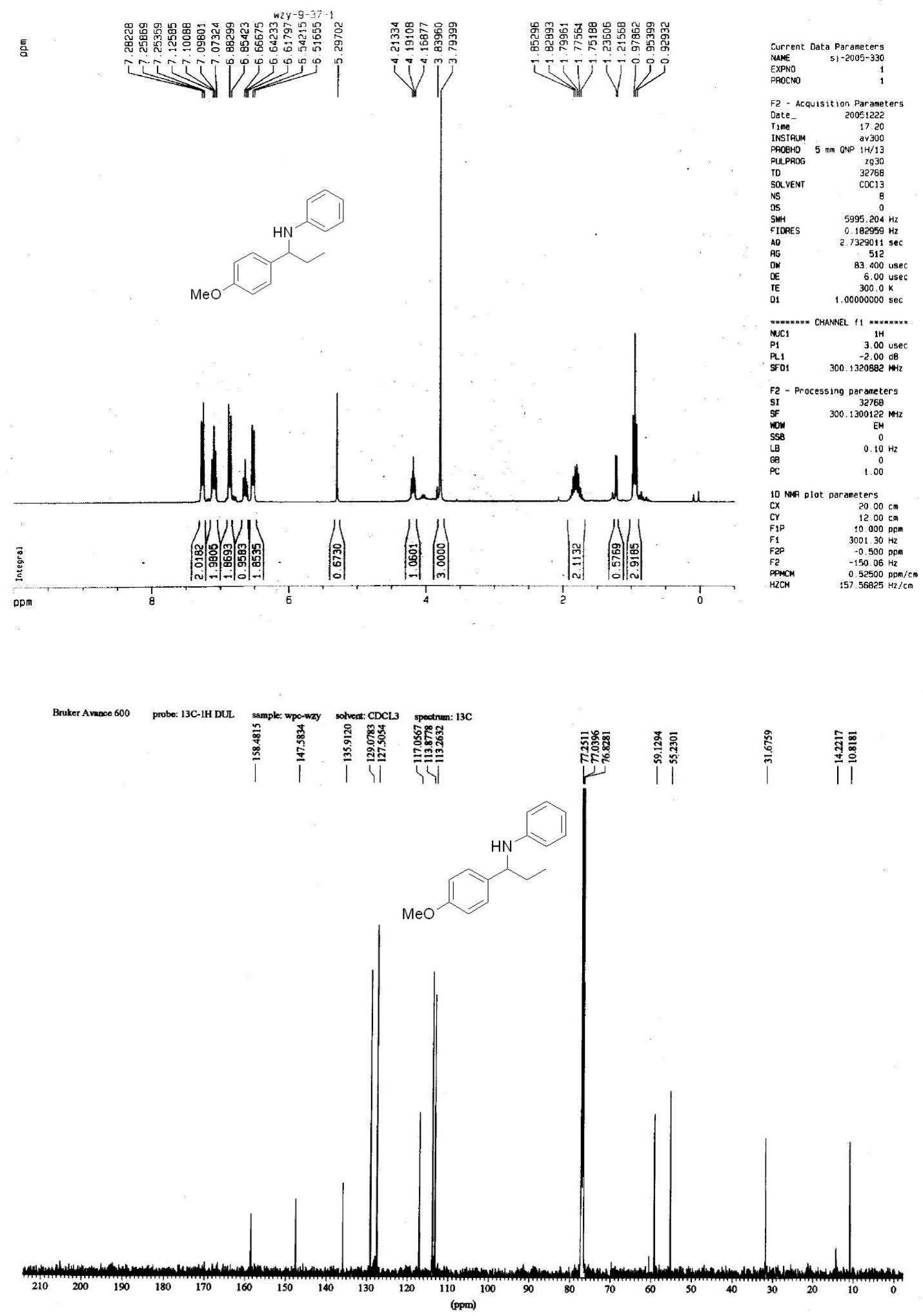
The NMR spectra of 7q
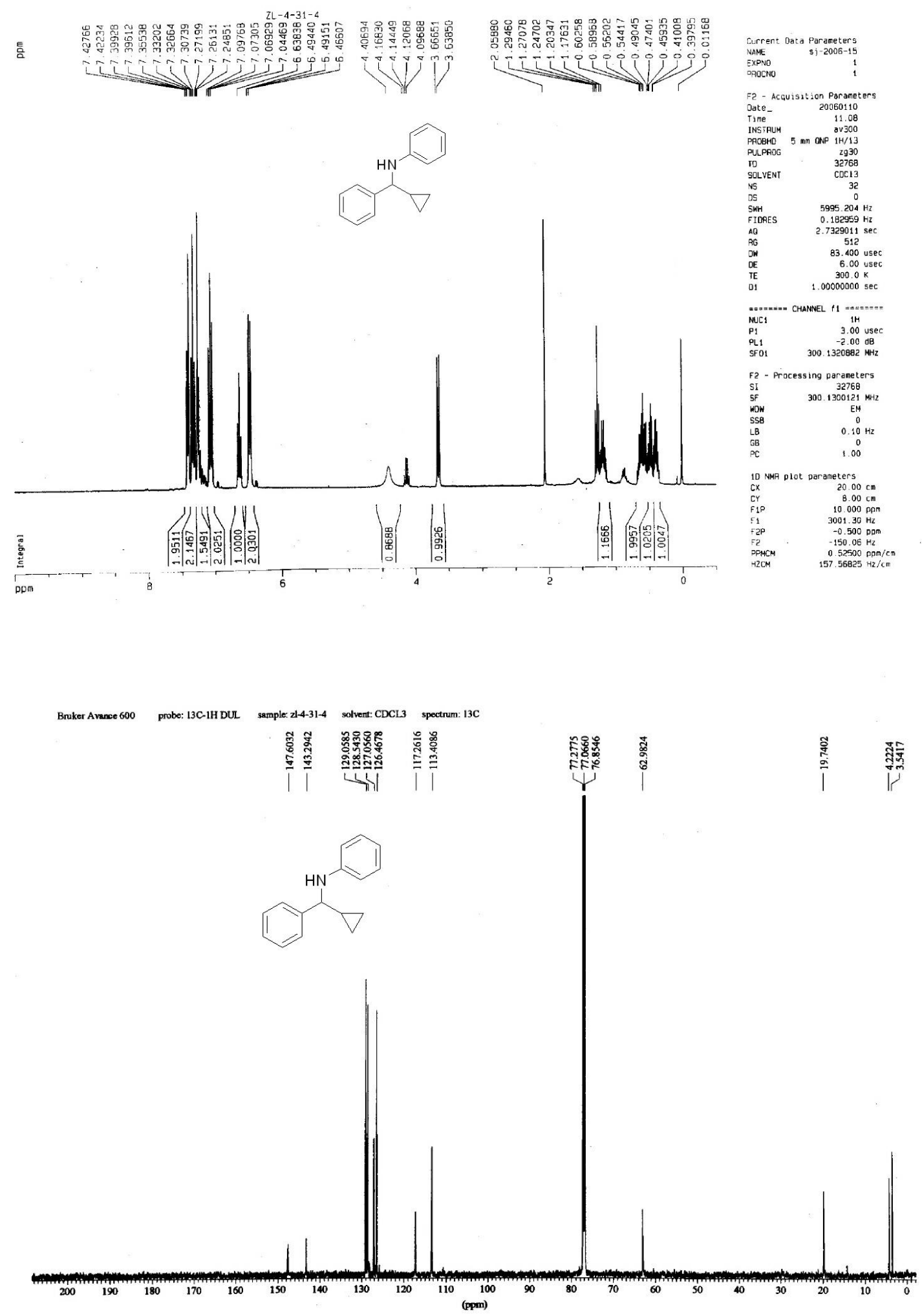
The NMR spectra of $\mathbf{7 r}$

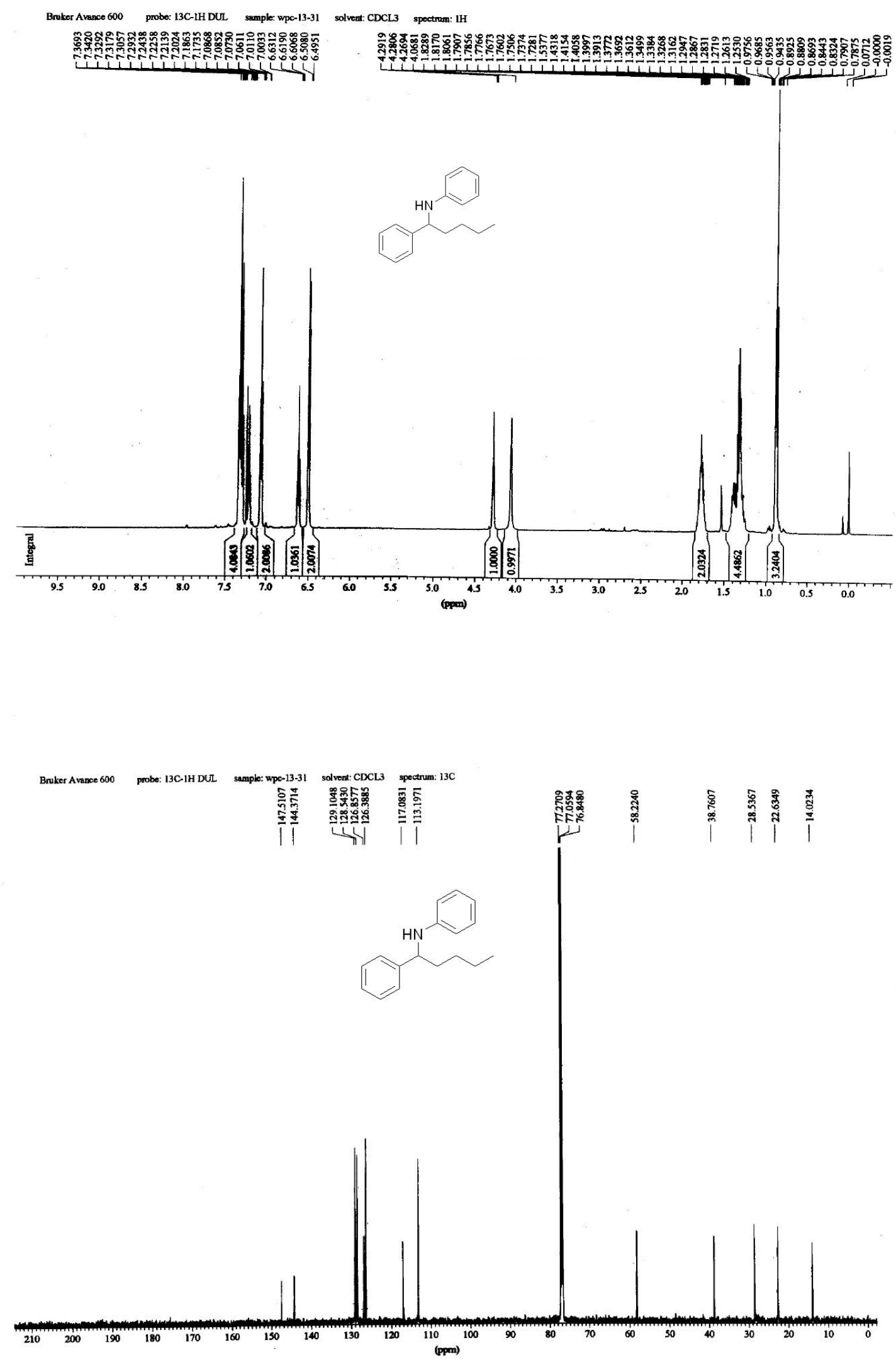


The NMR spectra of $7 \mathrm{~s}$

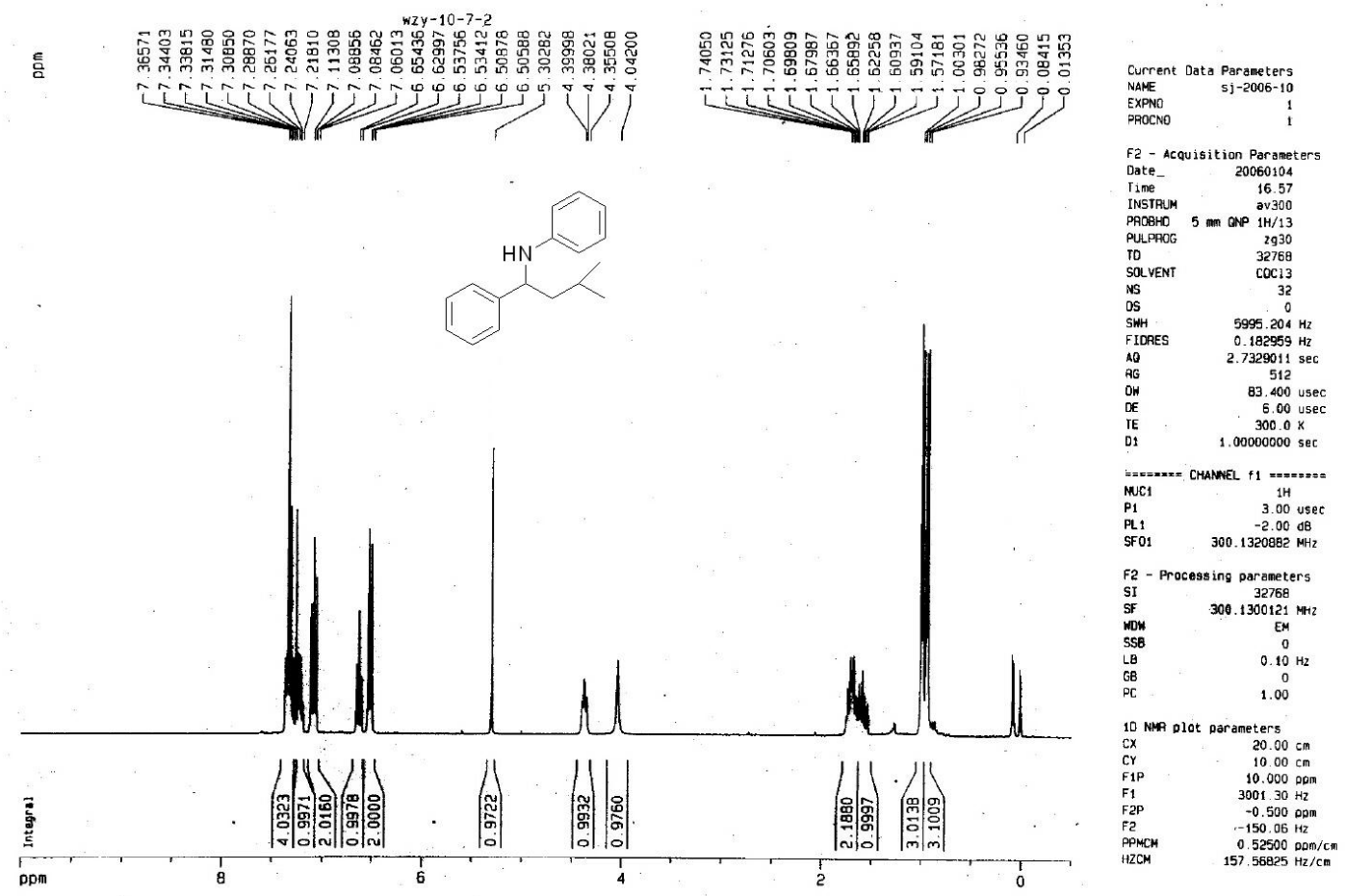

Bruker Avance 600 probe: $13 \mathrm{C}-1 \mathrm{H}$ DUL sample: wZy-10-7-2 solvent: CDCL3 spectrum: $13 \mathrm{C}$

\begin{tabular}{|c|c|c|c|c|}
\hline 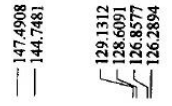 & 「。 & 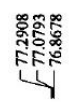 & 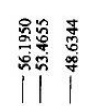 & 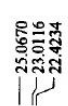 \\
\hline
\end{tabular}
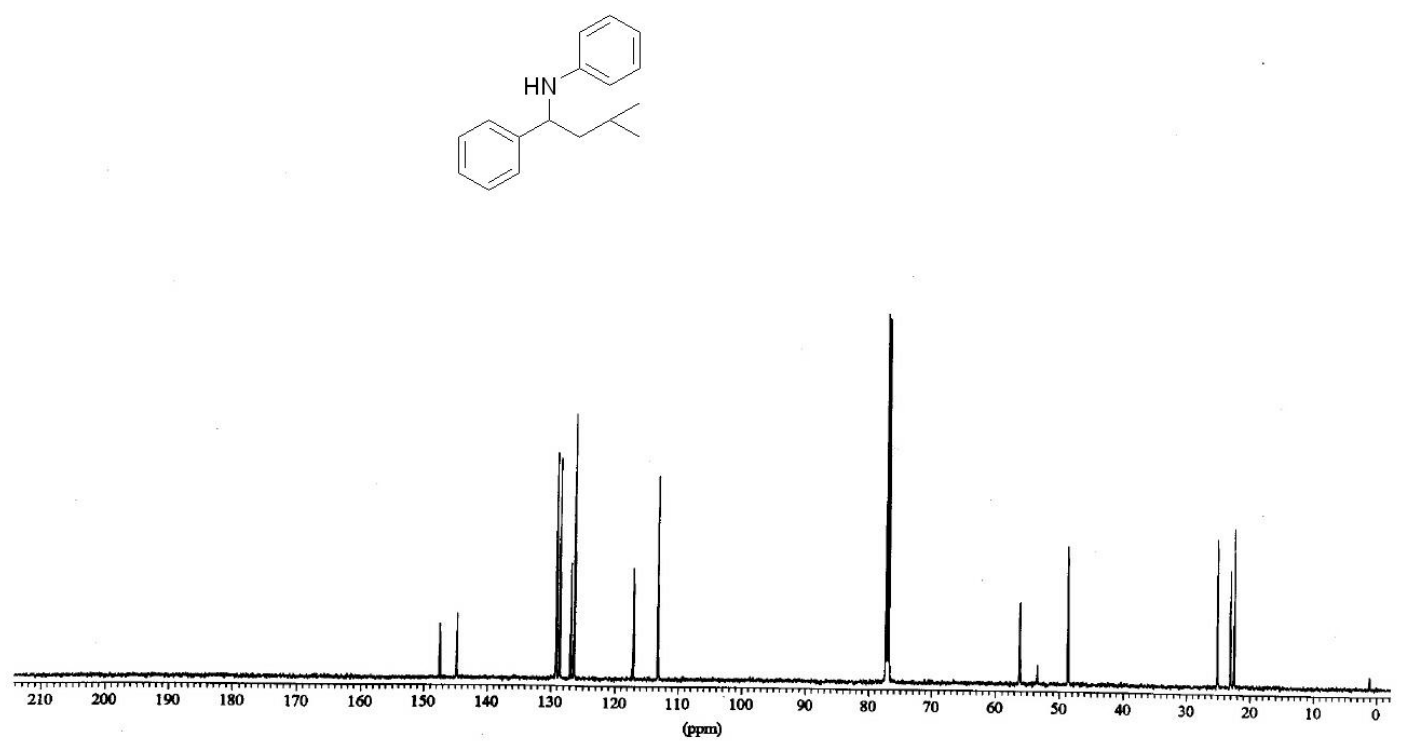
The HPLC spectra of $7 \mathbf{a}$

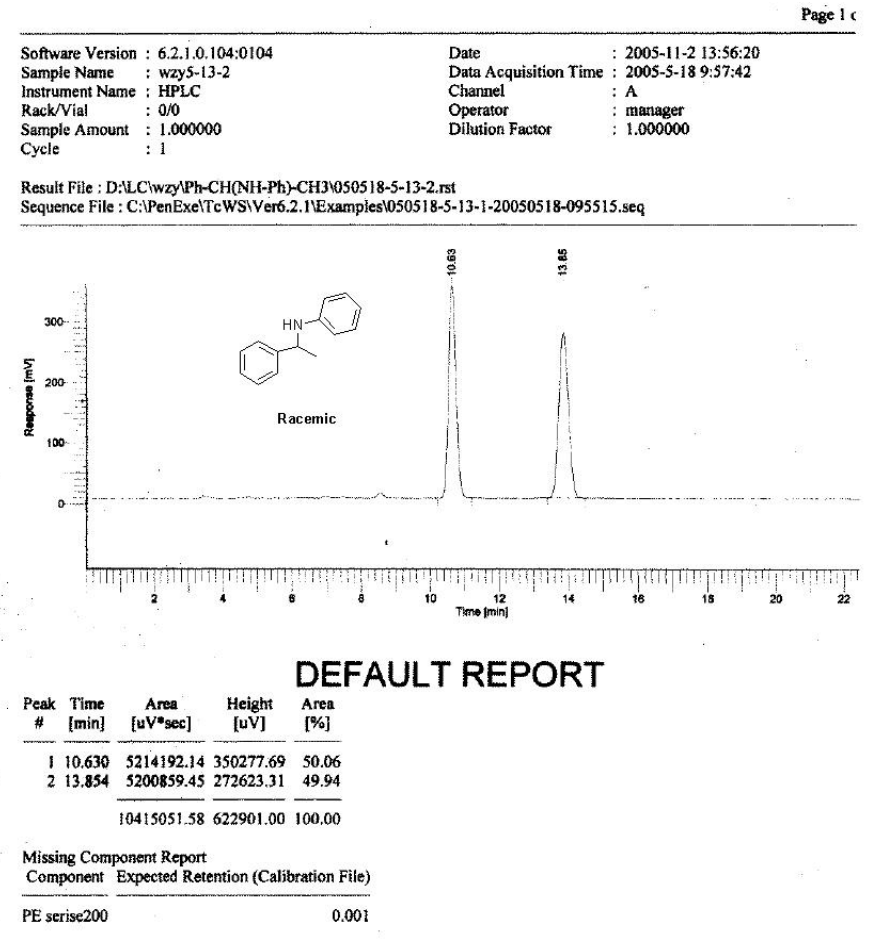

Time $\{$ minn

Page 1 of 1

\begin{tabular}{|c|c|c|c|c|}
\hline $\begin{array}{l}\text { Software Version } \\
\text { Sample Name } \\
\text { Instrument Name } \\
\text { Rack/Vial } \\
\text { Sample Amount } \\
\text { Cycle }\end{array}$ & $\begin{array}{l}: 6.2 .1 .0 .104: 0104 \\
\text { way11-4-1 } \\
: \text { HPLC } \\
: 0 / 0 \\
: 1.000000 \\
1\end{array}$ & & $\begin{array}{l}\text { Date } \\
\text { Data Acquisition Time } \\
\text { Channel } \\
\text { Operator } \\
\text { Dilution Factor }\end{array}$ & $\begin{array}{l}: \text { 2006-4-5 15:44:37 } \\
: 2006-4-5 \text { 15:18:34 } \\
: \text { A } \\
: \text { manager } \\
: 1.000000\end{array}$ \\
\hline
\end{tabular}

Cycle

Result File : D:LCCWzylPh-CH(NH-Ph)-CH31060405-11-4-1.rst

Sequence File : C:PenExelTc WSIVer6.2.1VExamplesl060405-11-4-1.seq

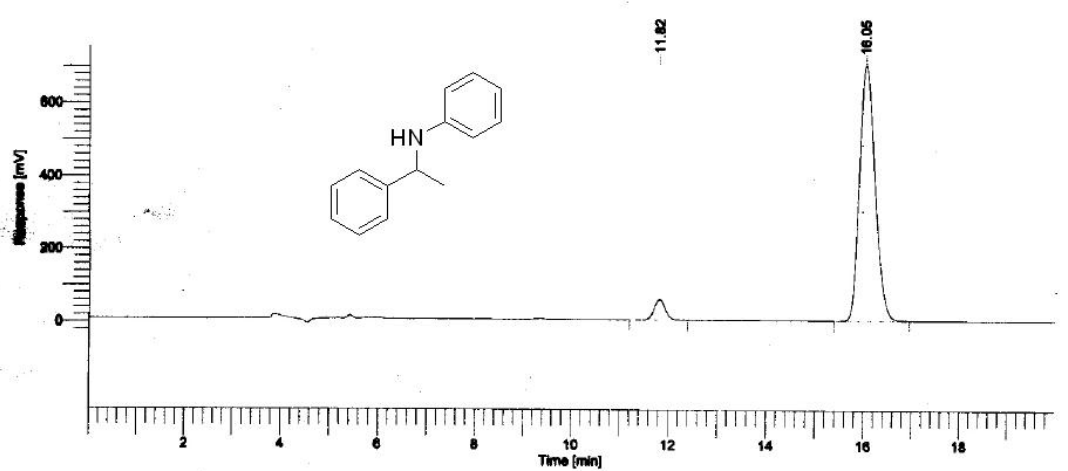

\section{DEFAULT REPORT}

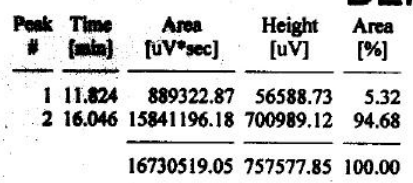

Missing Component Report

Component Expocted Retention (Calibration File)

PE series200

0.001 
The HPLC spectra of $\mathbf{7 b}$

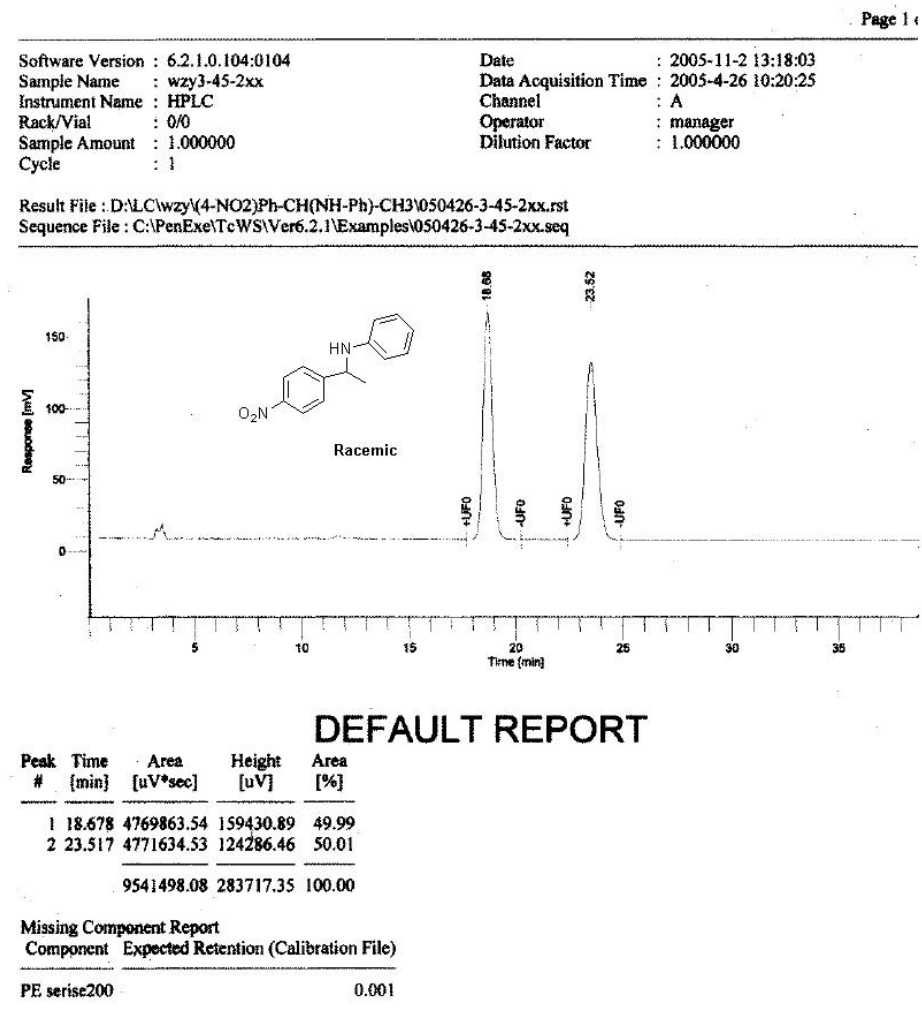

Page 1 of 1

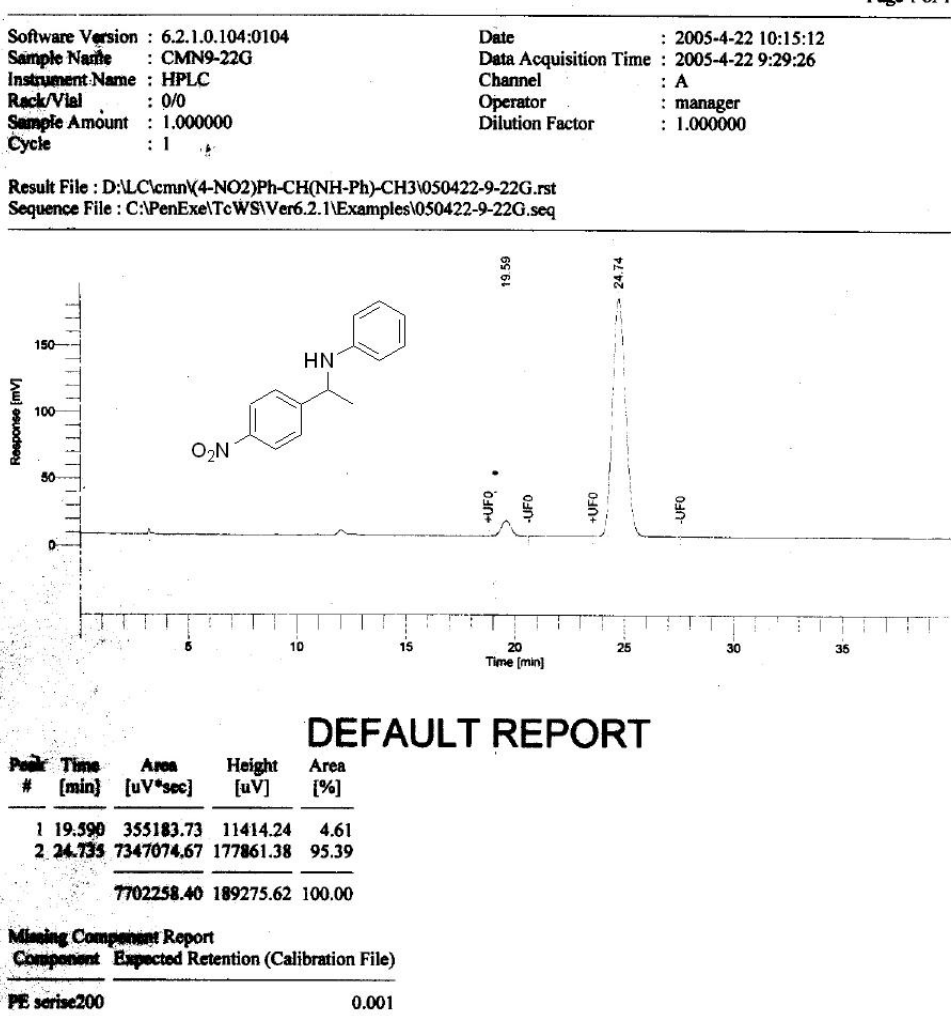


The HPLC spectra of $\mathbf{7 c}$

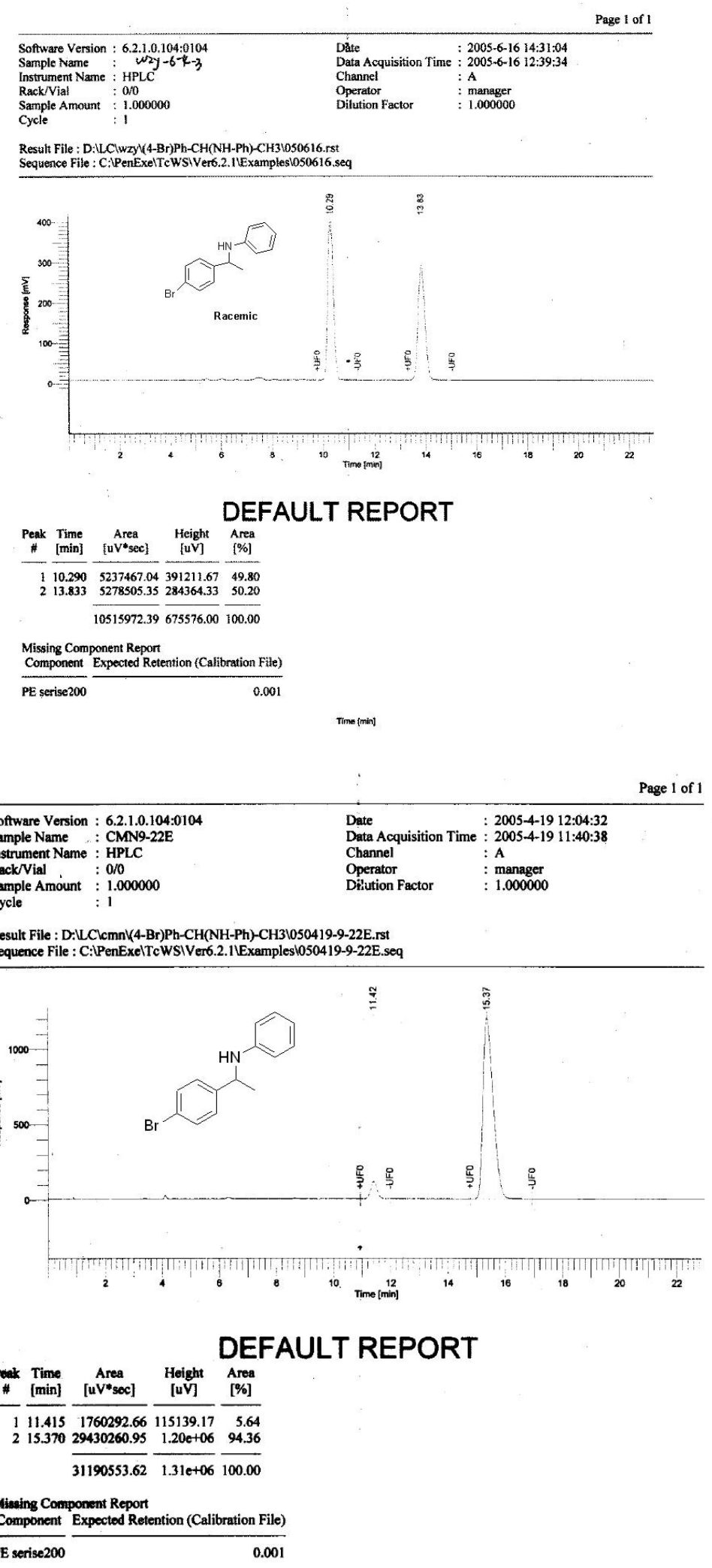


The HPLC spectra of 7d

\begin{tabular}{|c|c|c|c|c|}
\hline $\begin{array}{l}\text { Software Version } \\
\text { Sample Name } \\
\text { Instrument Name } \\
\text { Rack/Vial } \\
\text { Sample Amotnt } \\
\text { Cycle }\end{array}$ & $\begin{array}{l}: \text { 6.2.1.0.104:0104 } \\
\vdots \\
: \text { HPLC } \\
: 0 / 0 \\
: 1.000000 \\
: 1\end{array}$ & $\begin{array}{l}\text { Date } \\
\text { Data Acquisition Time } \\
\text { Channel } \\
\text { Operator } \\
\text { Dilution Factor }\end{array}$ & $\begin{array}{l}: 2006-4-14 \text { 13:52:57 } \\
: 2006-4-14 \text { 13:29:32 } \\
: \text { A } \\
: \text { manager } \\
: 1.000000\end{array}$ & \\
\hline \multicolumn{5}{|c|}{$\begin{array}{l}\text { Result File : D:LLClwzy)(4-CH3)Ph-CH(NH-Ph)-CH31060414-11-8-3xx-od1.rst } \\
\text { Sequence File : C:IPenExelTcWSIVer6.2.11Examplesi060414-11-8-3xx-od1.seq }\end{array}$} \\
\hline
\end{tabular}

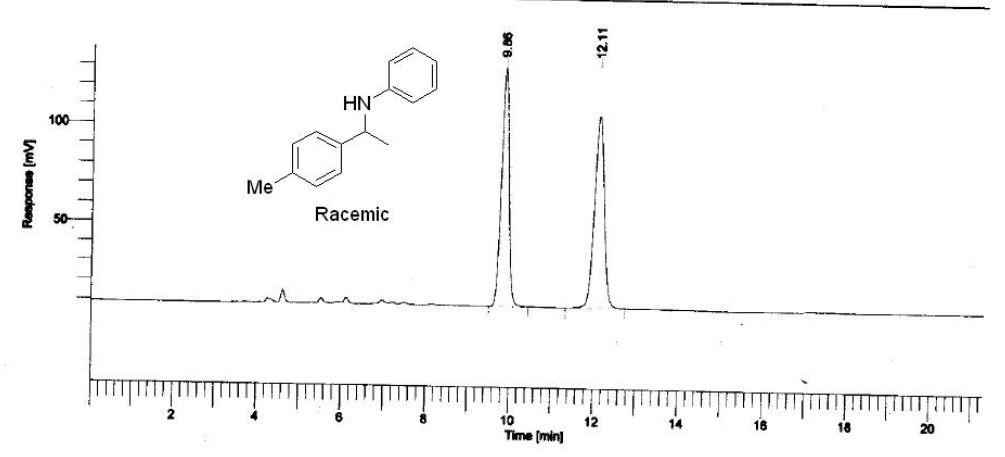

\section{DEFAULT REPORT}

\begin{tabular}{|c|c|c|c|c|}
\hline $\begin{array}{c}\text { Peak } \\
\#\end{array}$ & $\begin{array}{l}\text { Time } \\
\text { [min] }\end{array}$ & $\begin{array}{c}\text { Area } \\
{\left[u \mathrm{u}^{*} \mathrm{sec}\right]}\end{array}$ & $\begin{array}{l}\text { Height } \\
\text { [uV] }\end{array}$ & $\begin{array}{l}\text { Area } \\
{[\%]}\end{array}$ \\
\hline \multirow{2}{*}{\multicolumn{2}{|c|}{$\begin{array}{lr}1 & 9.85 \\
2 & 12.110\end{array}$}} & $\begin{array}{l}1551290.76 \\
1577724.35\end{array}$ & $\begin{array}{r}122138.70 \\
97986.90\end{array}$ & $\begin{array}{l}49.58 \\
50.42\end{array}$ \\
\hline & & 3129015.11 & 220125.60 & 100.00 \\
\hline \multicolumn{5}{|c|}{$\begin{array}{l}\text { Missing Component Report } \\
\text { Component Expected Reter }\end{array}$} \\
\hline & & & & \\
\hline
\end{tabular}

\begin{tabular}{|c|c|c|c|}
\hline $\begin{array}{l}\text { Software Version : } \\
\text { Sample Name } \\
\text { Instrument Name } \\
\text { Rack/Vial } \\
\text { Sample Amount } \\
\text { Cycle. }\end{array}$ & $\begin{array}{l}: 6.2 \cdot 1.0 .104: 0104 \\
: \text { wzy-11-8-1 } \\
: \text { HPLC } \\
: 0 / 0 \\
: 1.000000 \\
: 1\end{array}$ & $\begin{array}{l}\text { Date } \\
\text { Data Acquisition Time } \\
\text { Channel } \\
\text { Operator } \\
\text { Dilution Factor }\end{array}$ & $\begin{array}{l}: 2006-4-18 \text { 15:47:03 } \\
: 2006-4-1815: 30: 09 \\
: \text { A } \\
: \text { manager } \\
: 1.000000\end{array}$ \\
\hline
\end{tabular}
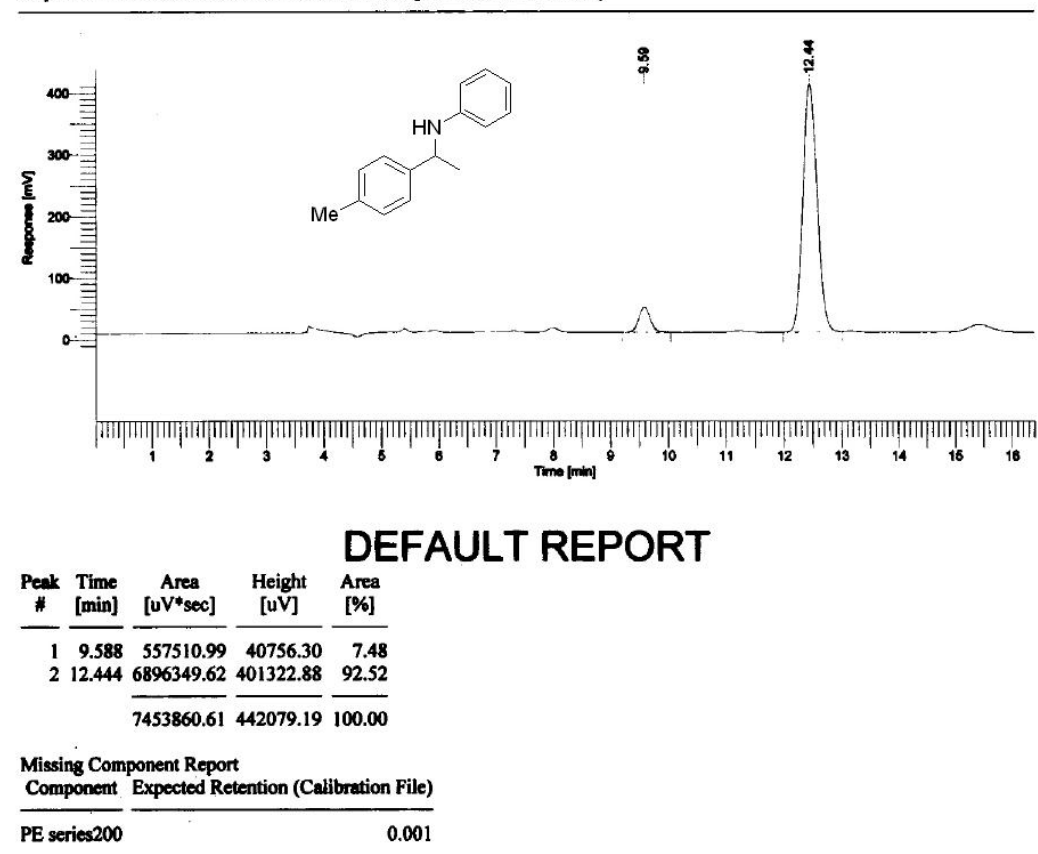
The HPLC spectra of $7 \mathbf{e}$
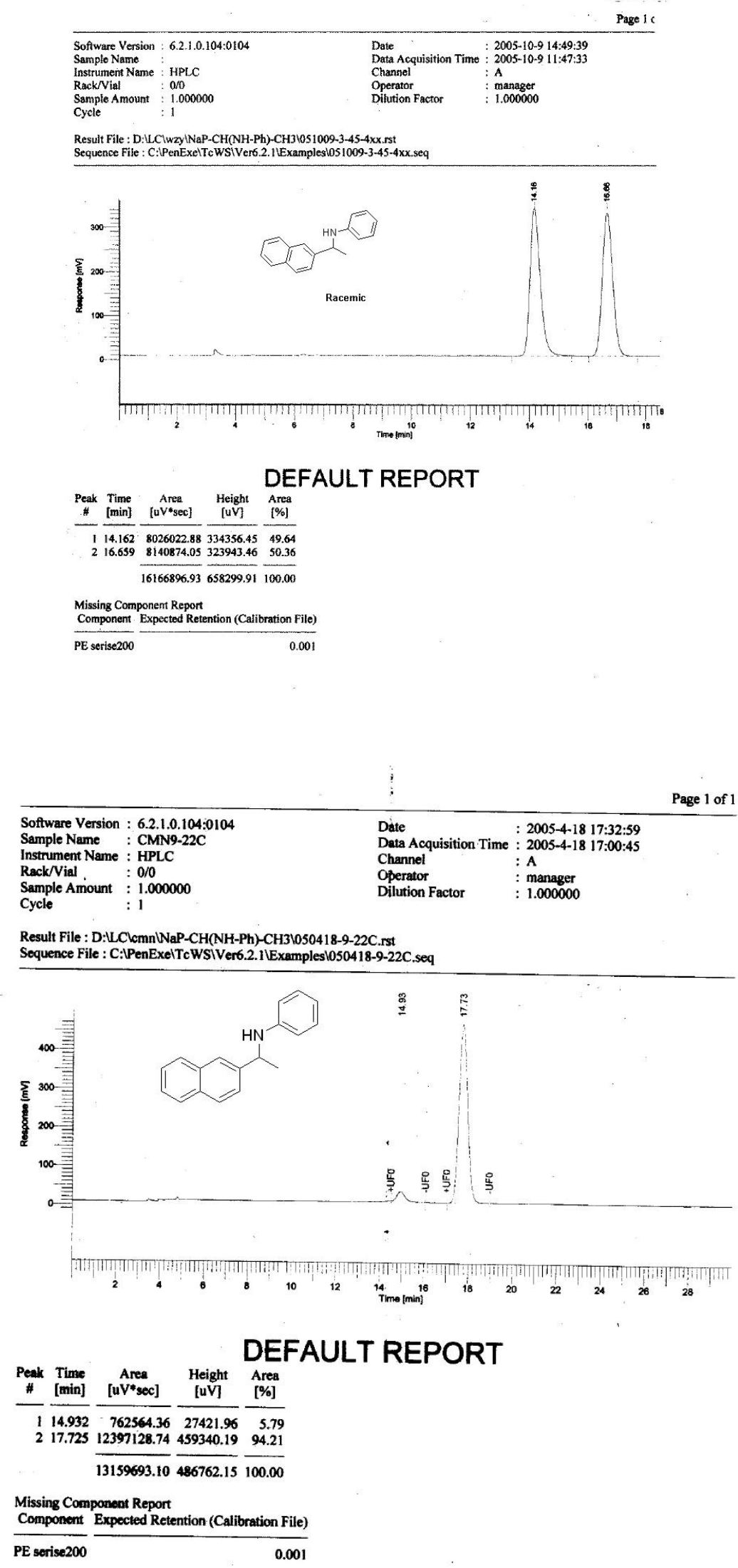
The HPLC spectra of $7 \mathbf{f}$

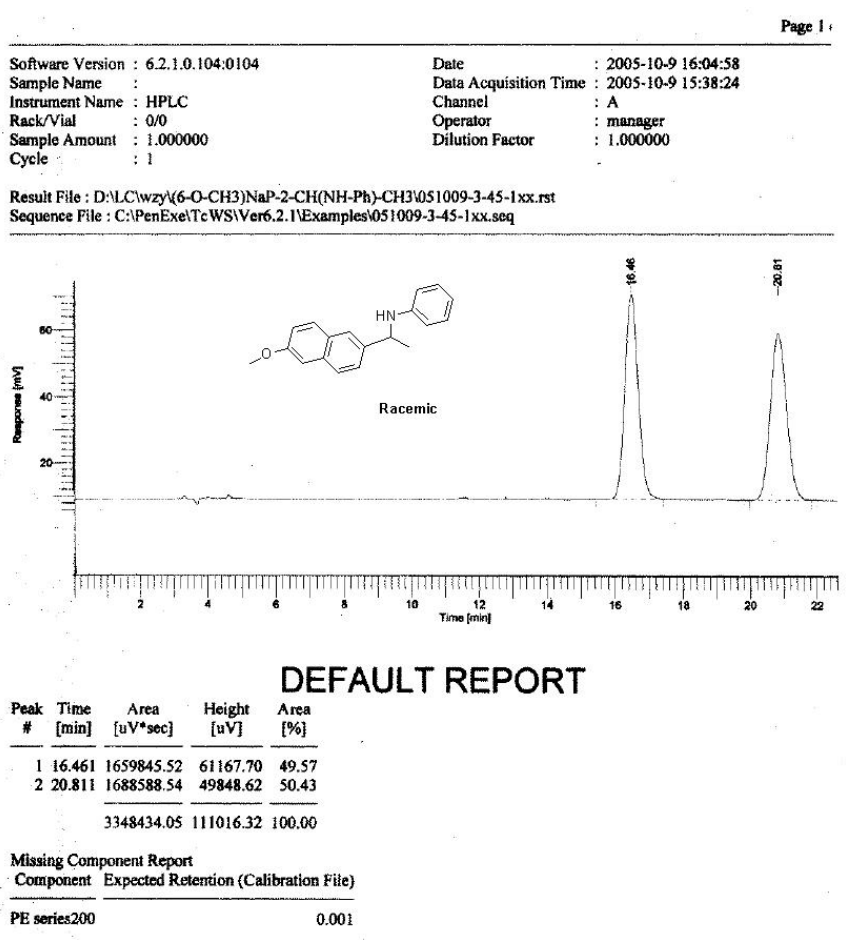

Software Version : 6.2.1.0.104:0104

Sample Name : wzy10-45-3

Instrument Name : HPLC

Rack/Vial $: 0 / 0$

Sample Amount : 1.000000

1

Date : 2006-3-31 18:07:16

Data Acquisition Time : 2006-3-31 16:54:04

Channel :A

$\begin{array}{ll}\text { Operator } & : \text { manager } \\ \text { Dilution Factor } & : 1.000000\end{array}$

Cycle

Result File : D:LLClwzy)(6-O-CH3)NaP-2-CH(NH-Ph)-CH31060331-10-45-3.rst

Sequence File : C:PenExelTcWSIVer6.2.1 1 Examples 1060331-10-45-3.seq

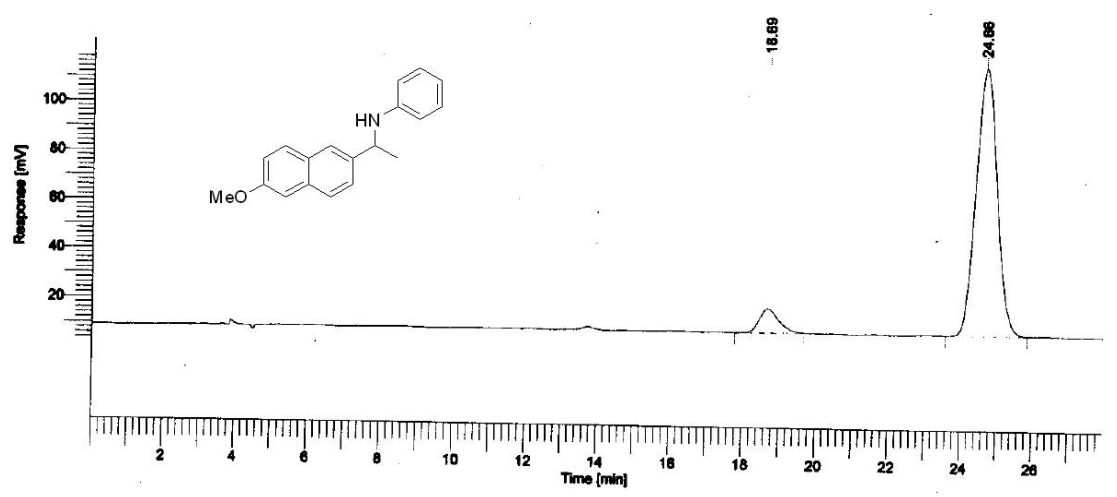

\section{DEFAULT REPORT}

$$
\begin{aligned}
& \begin{array}{cccc}
\begin{array}{c}
\text { Peak Time } \\
\#
\end{array}\left[\begin{array}{c}
\text { Area } \\
{[\mathrm{min}]}
\end{array}\right. & \begin{array}{c}
\text { Height } \\
{\left[\mathrm{uV}^{*} \mathrm{sec}\right]}
\end{array} & \begin{array}{c}
\text { Area } \\
{[\mathrm{uV}]}
\end{array} & {[\%]}
\end{array} \\
& \text { I } \overline{18.692} \overline{352775.87} \overline{9650.12} \overline{7.41} \\
& 224.660 \quad 4405254.71 \quad 108680.88 \quad 92.59 \\
& 4758030.59118331 .00 \quad 100.00
\end{aligned}
$$

Missing Component Report

Component Expectod Retention (Calibration File)

PE series200

0.001 
The HPLC spectra of $\mathbf{7 g}$

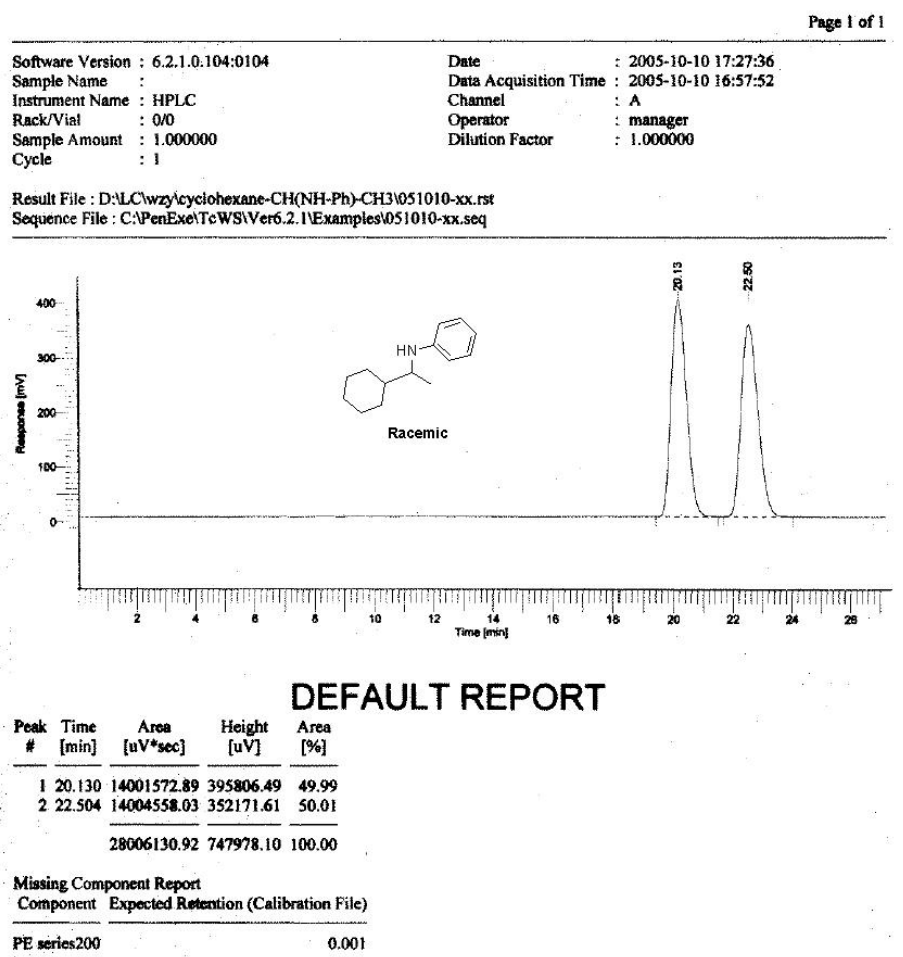

Page 1 of 1

\begin{tabular}{llll}
\hline Software Version $: 6.2 .1 .0 .104: 0104$ & Date & $: 2005-9-3016: 31: 27$ \\
Seanple Name : wzys-29-3 & Data Acquisition Time & $: 2005-9-30$ 16:02:21 \\
Instrument Name & $:$ HPLC & Channel & $:$ A \\
Rack/Vial & $: 010$ & Operator & $:$ manager \\
Sample Amount & $: 1.000000$ & Dilution Factor & $: 1.000000$ \\
Cycle & $: 1$ & &
\end{tabular}

Result File : D:LClwzyleyclohexane-CH(NH-Ph)-CH3l050930-8-29-3.rst

Sequence File: C:IPenExelTcWSIVer6.2.IExamples 1050930-8-29-3.seq

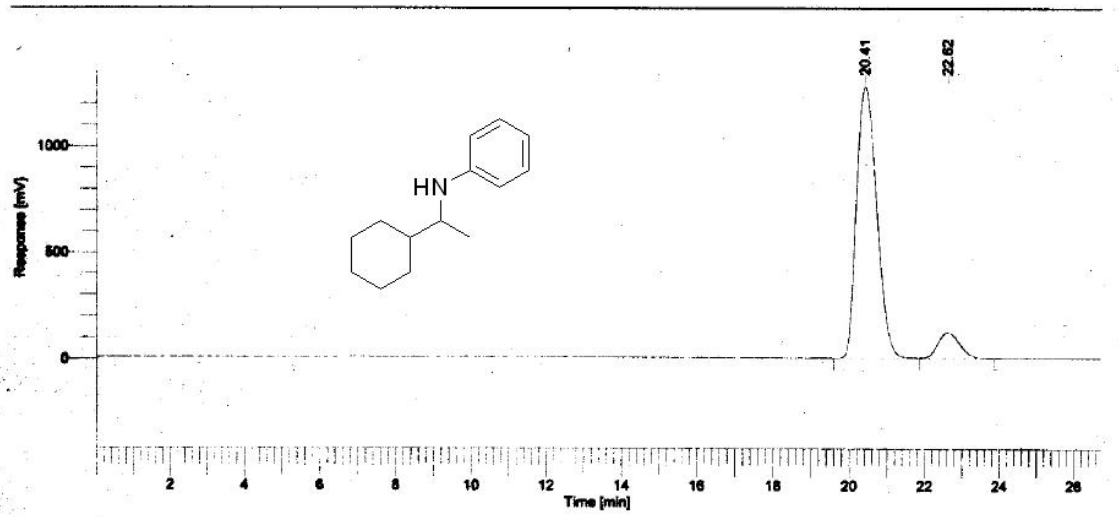

\section{DEFAULT REPORT}

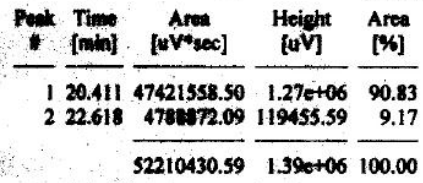

Miasing Componem Report

Component Expocted Retention (Calibration File)

PE serise200 
The HPLC spectra of $\mathbf{7 h}$

Page 1 of 1

\begin{tabular}{|c|c|c|c|}
\hline $\begin{array}{l}\text { Software Vention } \\
\text { Sample Name } \\
\text { Instrument Name } \\
\text { Rack/Vial } \\
\text { Sample Amouint } \\
\text { Cycle }\end{array}$ & $\begin{array}{l}: 6.2 .1 .0 .104: 0104 \\
: \\
: \text { HPLC } \\
: 0 / 0 \\
: 1.000000 \\
: 1\end{array}$ & $\begin{array}{l}\text { Date } \\
\text { Data Acquisition Time } \\
\text { Channel } \\
\text { Operator } \\
\text { Dilution Factor }\end{array}$ & $\begin{array}{l}: \text { 2006-3-30 11:19:08 } \\
: 2006-3-3011: 01: 07 \\
: \text { A } \\
: \text { manager } \\
: 1.000000\end{array}$ \\
\hline \multicolumn{4}{|c|}{$\begin{array}{l}\text { Result File : D:LCClwzylcyclohexane-CH[N(CHO)-Ph]-C2H51060330-xx-oj2.sst } \\
\text { Sequence File : C:PenExelTcWSIVer6.2.11Examples1060330-xx-0j2.seq }\end{array}$} \\
\hline
\end{tabular}

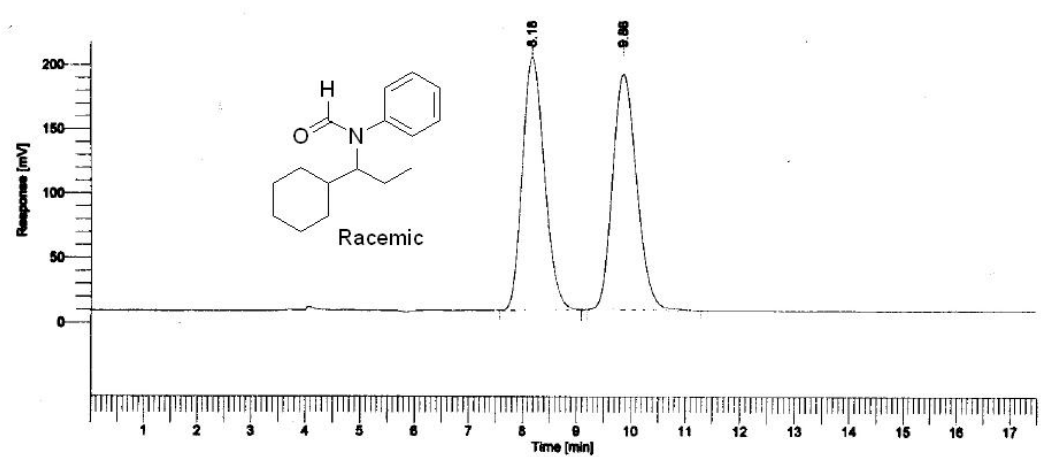

\section{DEFAULT REPORT}

$$
\begin{aligned}
& \begin{array}{c}
\text { Peak Time Area Height Area } \\
\text { \# [min] }
\end{array}
\end{aligned}
$$

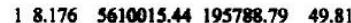

$$
\begin{aligned}
& 29.8645652918 .44 \\
& 11262934.08378675 .43 \quad 100.00
\end{aligned}
$$

Missing Component Report

Component Expected Retention (Calibration File)

PE series200

0.001

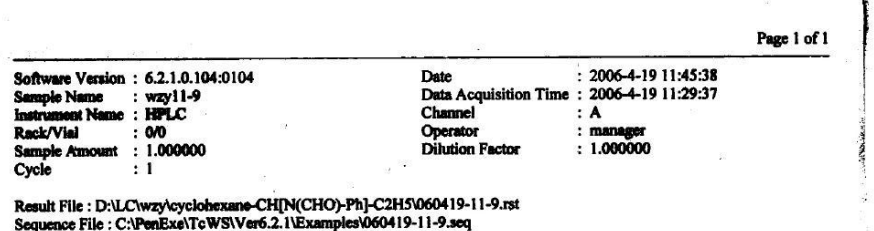

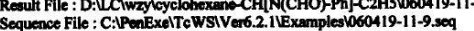

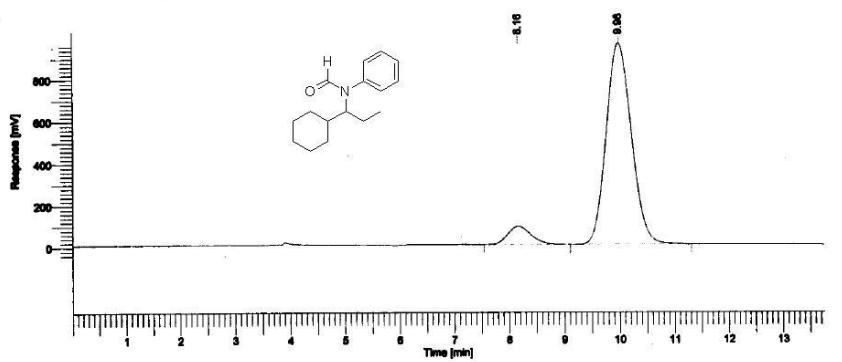

\section{DEFAULT REPORT}

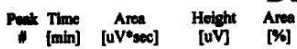

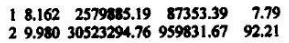

$\overline{33103179.95} \overline{1.05 e+06} \overline{100.00}$

Misuing Component Repont

Cxpectod Retention (Calibration Filo) 


\begin{tabular}{|c|c|c|c|}
\hline $\begin{array}{l}\text { Sottware Version } \\
\text { Sample Name } \\
\text { Instrument Name } \\
\text { Rack/Vial } \\
\text { Sample Amount } \\
\text { Cycle }\end{array}$ & $\begin{array}{l}: \text { : } 6.2 .1 .0 .104: 0104 \\
: \text { wZy11-2-2 } \\
: \text { HPLC } \\
: 0 / 0 \\
: 1.000000 \\
: 1\end{array}$ & $\begin{array}{l}\text { Date } \\
\text { Data Acquisition Time } \\
\text { Channel } \\
\text { Operator } \\
\text { Dilution Factor }\end{array}$ & $\begin{array}{l}\text { 2006-4-4 12:15:05 } \\
\text { 2006-4-4 11:57:36 } \\
: \text { A } \\
\text { manager } \\
1.000000\end{array}$ \\
\hline
\end{tabular}

Result File : D:LClwzylcyclohexane-CH[N(CHO)-Ph]-C2H51060404-11-2-2.rst Sequence File : C:IPenExelTcWSIVer6.2.1Lxamples1060404-11-2-2.sea

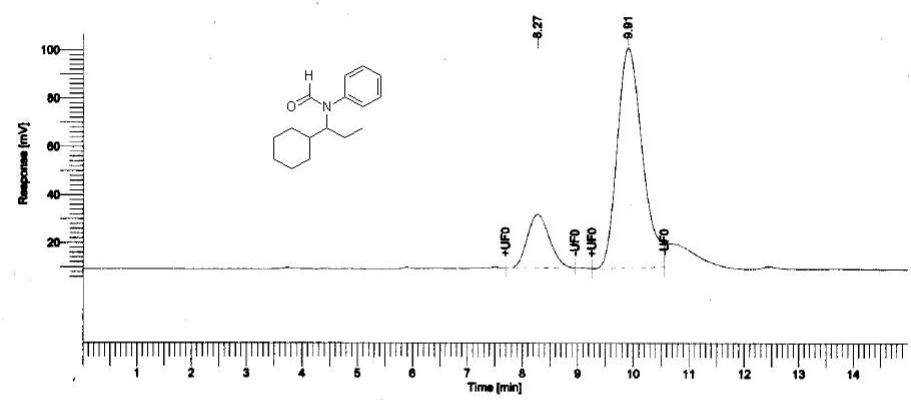

\section{DEFAULT REPORT}

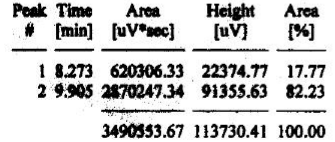

Missing Componeat Report

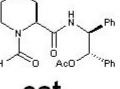

Component Expected Retention (Calibration File)

cat

PE series200

0.001 
The HPLC spectra of $7 \mathbf{i}$

\begin{tabular}{|c|c|c|c|c|}
\hline $\begin{array}{l}\text { Solwware Version } \\
\text { Sample Name } \\
\text { Inatrusueat Name } \\
\text { Rack/Viad } \\
\text { Sumple Amount } \\
\text { Cycle }\end{array}$ & $\begin{array}{l}: 6.2 .1 .0 .104: 0104 \\
: \text { wzy3-45-6xx } \\
: \text { HPLC } \\
: 0 / 0 \\
: 1.000000 \\
: 1\end{array}$ & $\begin{array}{l}\text { Date } \\
\text { Data Acquisition Time } \\
\text { Channel } \\
\text { Operator } \\
\text { Dilution Factor }\end{array}$ & $\begin{array}{l}: 2006-4-1017: 13: 23 \\
: 2005-3-23 \quad 16: 45: 57 \\
: \text { A } \\
: \text { manager } \\
: 1.000000\end{array}$ & \\
\hline \multicolumn{5}{|c|}{$\begin{array}{l}\text { Resul File : D.LClwzy/Ph-CH(NH-Ph)-C2H51050323-3-45-6xx.rst } \\
\text { Sequence File : C:PenExelTcWSIVer6.2.11Examples1050323-3-45-6xx.seq }\end{array}$} \\
\hline
\end{tabular}

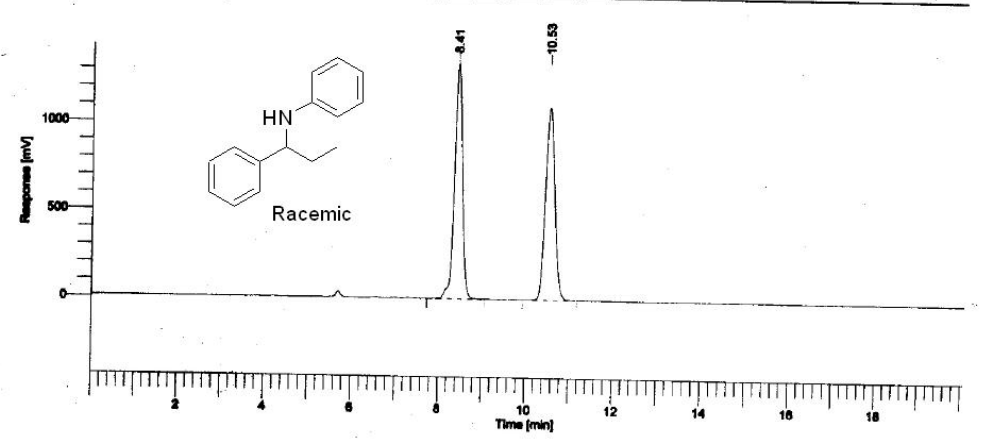

\section{DEFAULT REPORT}

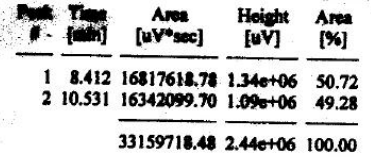

Misesing Componeen Repport

\begin{tabular}{|c|c|c|c|c|}
\hline & & & & Page 1 of 1 \\
\hline $\begin{array}{l}\text { Software Version } \\
\text { Sample Name } \\
\text { Instrument Name } \\
\text { Rack/Vial } \\
\text { Sample Amount } \\
\text { Cycle }\end{array}$ & $\begin{array}{l}\text { 6.2.1.0.104:0104 } \\
: \text { CMN9-22M } \\
: \text { HPLC } \\
: 0 / 0 \\
: 1.000000 \\
: 1\end{array}$ & $\begin{array}{l}\text { Date } \\
\text { Data Acquisition Time } \\
\text { Channel } \\
\text { Operator } \\
\text { Dilution Factor }\end{array}$ & $\begin{array}{l}: 2006-4-1017: 17: 15 \\
: 2005-4-18 \text { 11:13:24 } \\
: \text { A } \\
: \text { manager } \\
: 1.000000\end{array}$ & \\
\hline
\end{tabular}

Component Expodted Retention (Calibration File)

FE serise200

0.001

Result File : D:LClemn(Ph-CH(NH-Ph)-C2H51050418-9-22M.rst

Sequence File : C:PenExelTcWSIVer6.2.11Examplesl050418-9-22M.seq

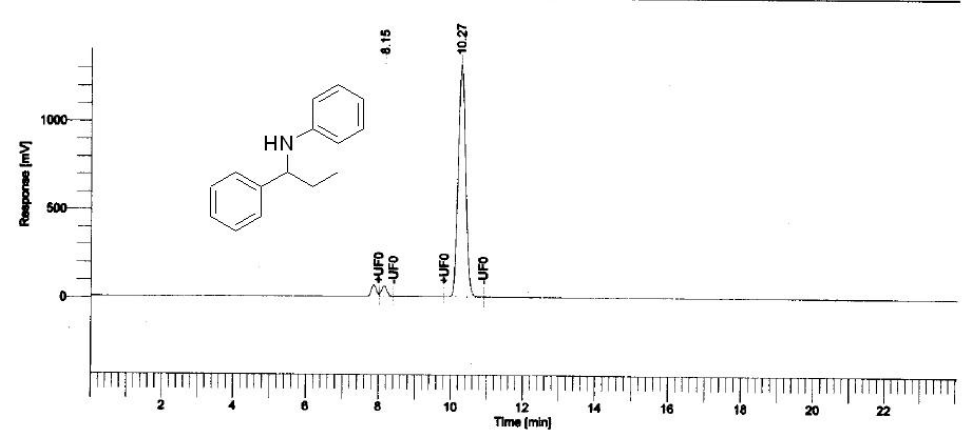

\section{DEFAULT REPORT}

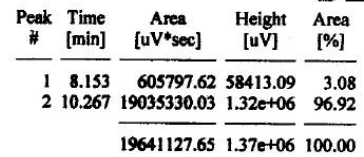

Missing Component Report

Component Expected Retention (Calibration File)

PE serise200
0.001 
Software Version : 6.2.1.0.104:0104

Sample Name : wzy8-33-1

Rack/Vial $:$ : $0 / 0$

Rack/Vial

$\begin{array}{ll}\text { Sample Amount } & : 1.000000 \\ \text { Cycle } & : 1\end{array}$

Result File : D:LClwzylPh-CH(NH-Ph)-C2H51051008-8-33-1.rst

Sequence File : C:PenExelTcWSIVer6.2.1 Examplesl051008-8-33-1.seq

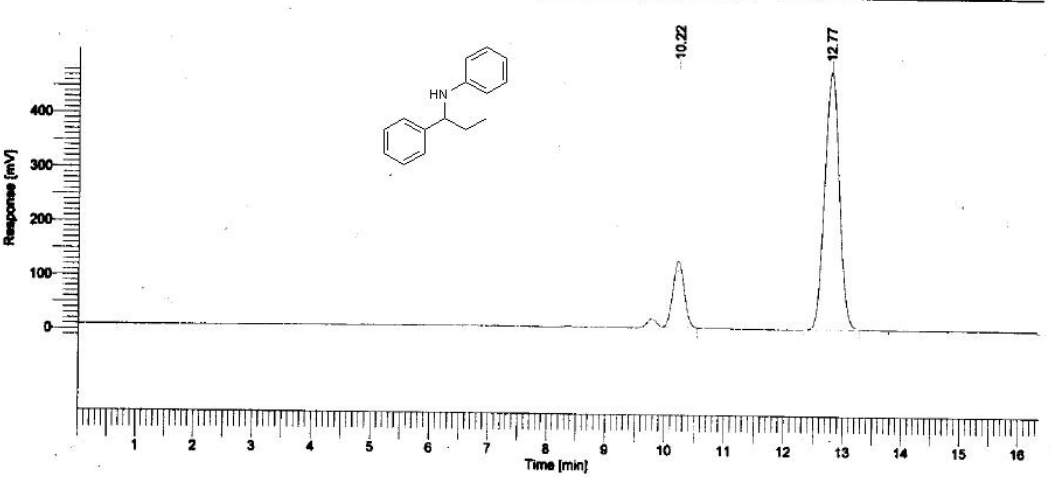

\section{DEFAULT REPORT}

Date : 2006-4-12 14:35:09

Channel

A

1.000000

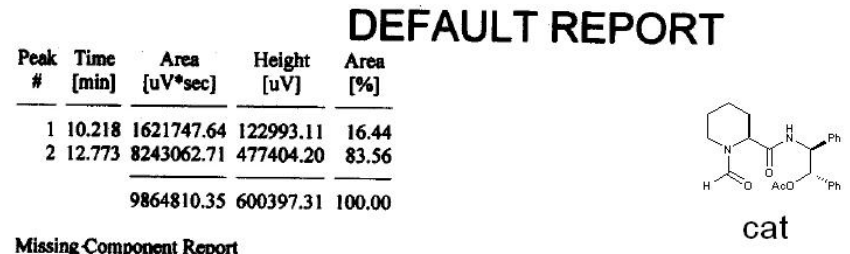

Missing Component Report

cat 
The HPLC spectra of $\mathbf{7 j}$
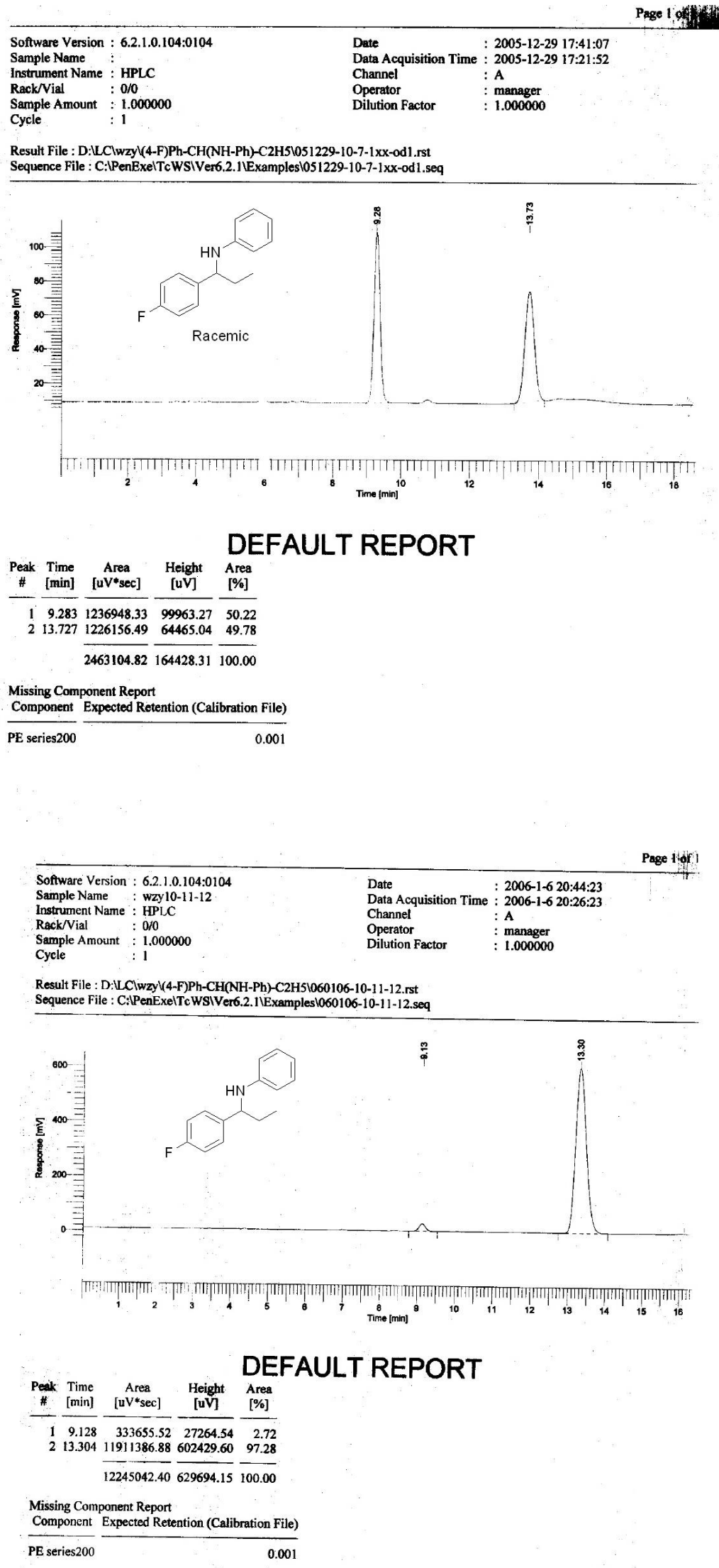
The HPLC spectra of $\mathbf{7 k}$

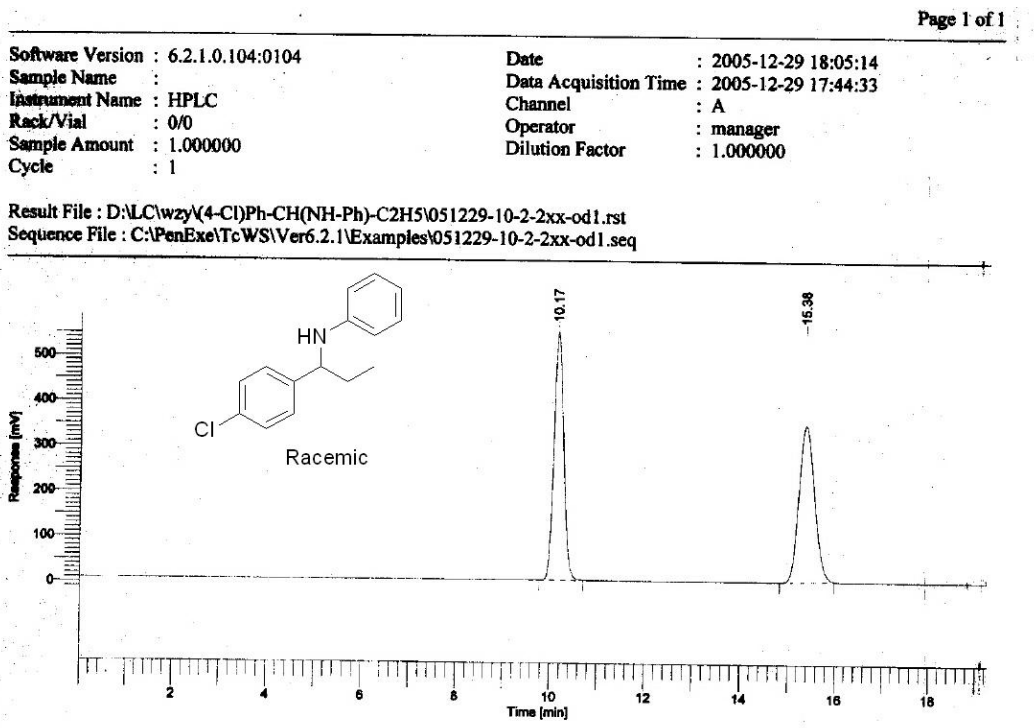

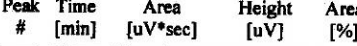

$$
\begin{aligned}
& \text { I } \overline{10.169} \overline{7814594.73} \frac{}{546377.16} \frac{[\%]}{49.85} \\
& \begin{array}{lllllll}
2 & 15.379 & 7861542.86 & 345193.61 & 50.15
\end{array} \\
& \overline{15676137.59} \overline{891570.77} \overline{100.00}
\end{aligned}
$$

DEFAULT REPORT

Missing Component Report

Component Expected Retention (Calibration File)

PE series200

0.001

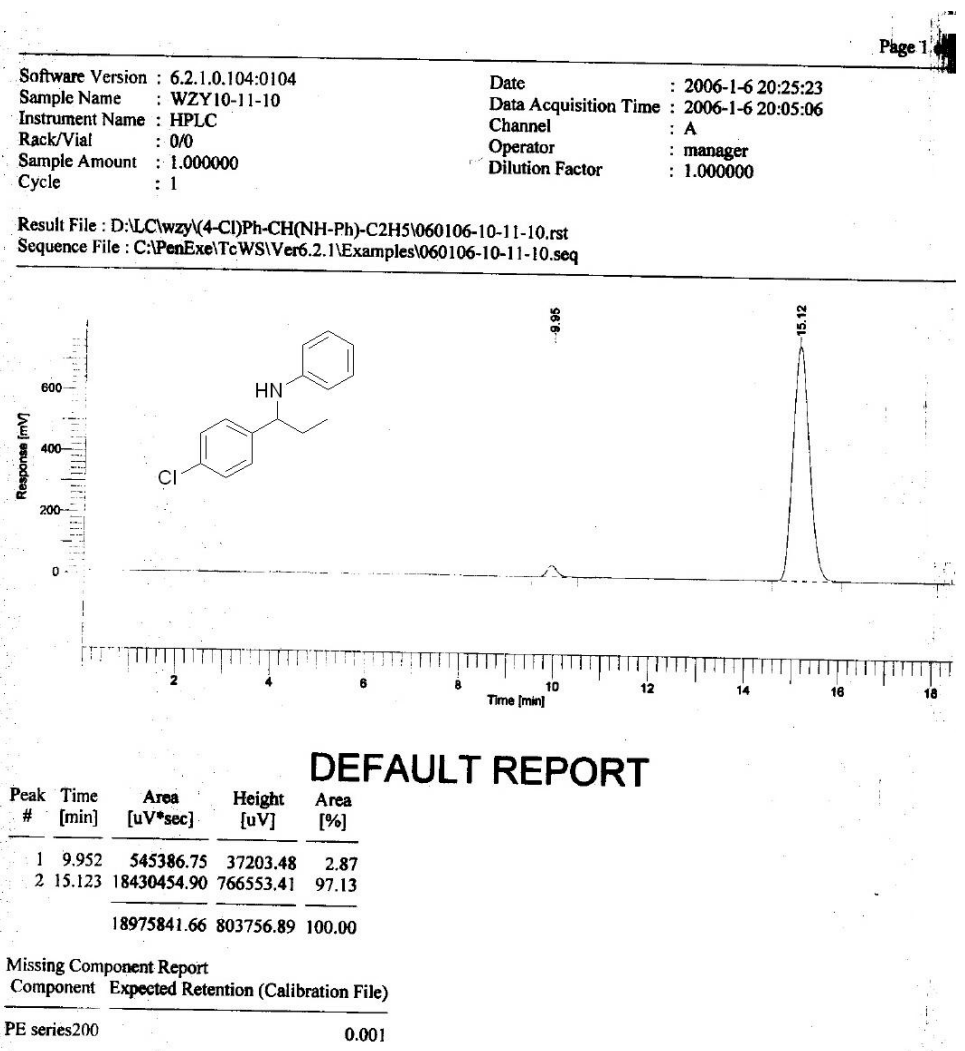


The HPLC spectra of 71

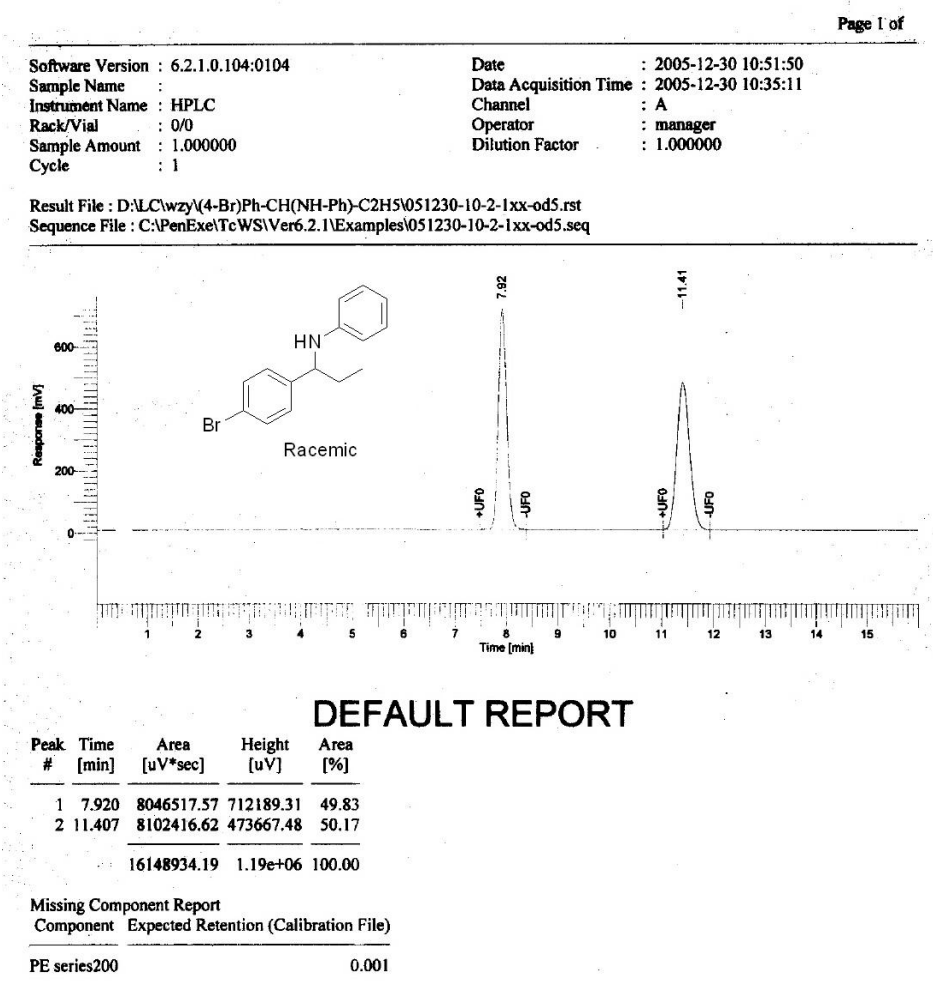

Page 10

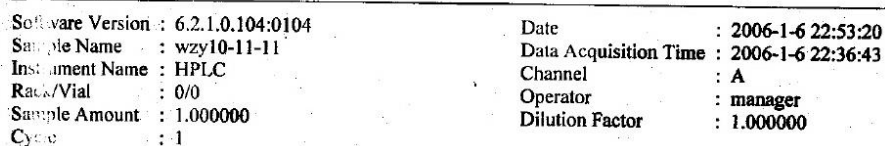

Sample Amount : 100000

Re it File : D:LCClwzyl(4-Br)Ph-CH(NH-Ph)-C2H51060106-10-1 1-11.rst

S. ence File : C; PenExelTcWSIVer6.2.1 Examples $1060106-10-11-11$.seq

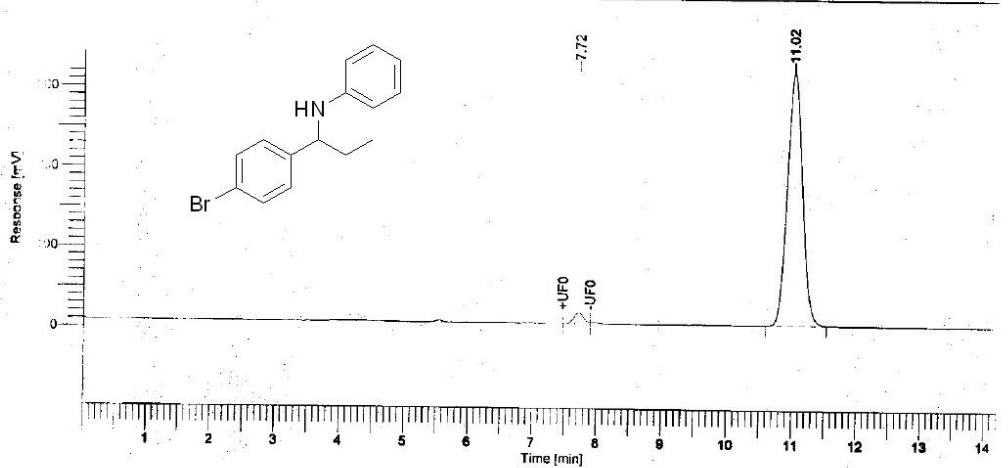

$$
\begin{aligned}
& \text { DEFAULT REPORT } \\
& \begin{array}{ccc}
\text { Pw Time Area Height Area } \\
{[\mathrm{min}]} \\
{\left[\mathrm{uV}^{*} \mathrm{sec}\right]}
\end{array} \\
& -\frac{[\mathrm{min}]}{7.720} \frac{[\mathrm{uV*} \text { sec] }}{[\mathrm{uV}]} \frac{\text { [\%] }}{271743.41} \\
& \overline{5244554.87} \overline{327926.85} \overline{100.00}
\end{aligned}
$$


The HPLC spectra of $7 \mathbf{m}$

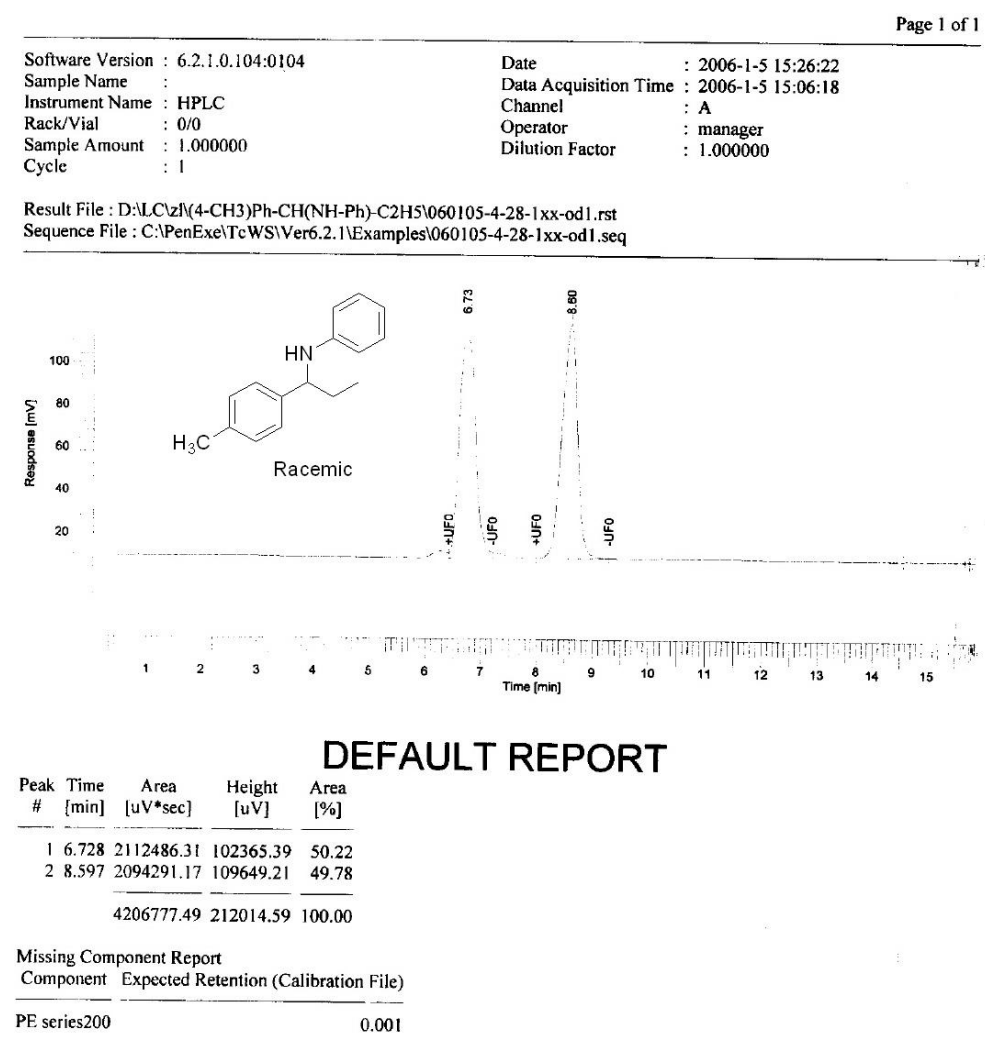

Software Version : 6.2.1.0.104:0104

Sample Name : wzy10-11-9

Rack/Vial $: 0 / 0$

$\begin{array}{ll}\text { Sample Amount } & : 1.000000 \\ \text { Cycle } & : 1\end{array}$

$\begin{array}{ll}\text { Date } & : 2006-1-6 \\ \text { Data Acquisition Time }: 2006-1-6 & 19: 25: 09\end{array}$

Data Acquisition Time :
Channel

Operator $\quad:$ A manager

$106-10-11-9.5 s t$

Sequence File : C.PenExelTcWSTVer6.2. NExamples $1060106-10-11-9$ seq

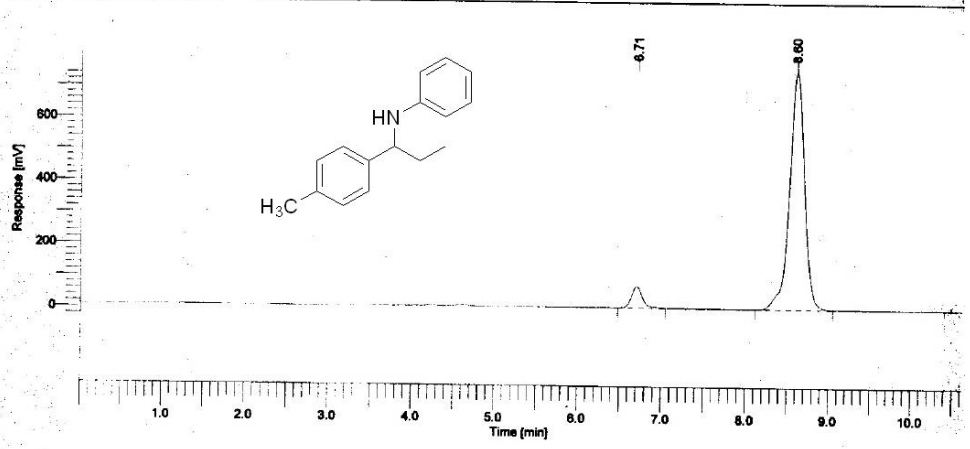

\section{DEFAULT REPORT}

\begin{tabular}{|c|c|c|c|}
\hline $\begin{array}{l}\text { Peak Time } \\
\# \text { [min] }\end{array}$ & $\begin{array}{c}\text { Area } \\
{\left[\mathrm{uV}^{*} \mathrm{sec}\right]}\end{array}$ & $\begin{array}{c}\text { Height } \\
{[\mathrm{uV}]}\end{array}$ & $\begin{array}{l}\text { Area } \\
{[\%]}\end{array}$ \\
\hline $\begin{array}{l}16.706 \\
28.602\end{array}$ & $\begin{array}{r}609566.64 \\
9363936.21\end{array}$ & $\begin{array}{r}66713.34 \\
747328.86\end{array}$ & $\begin{array}{r}6.11 \\
93.89\end{array}$ \\
\hline & 9973502.85 & 814042.20 & 100.00 \\
\hline $\begin{array}{l}\text { Missing Com } \\
\text { Component }\end{array}$ & $\begin{array}{l}\text { mponent Repo } \\
\text { Expected } \mathbf{R}\end{array}$ & ott & alibration File) \\
\hline
\end{tabular}


The HPLC spectra of $\mathbf{7 n}$

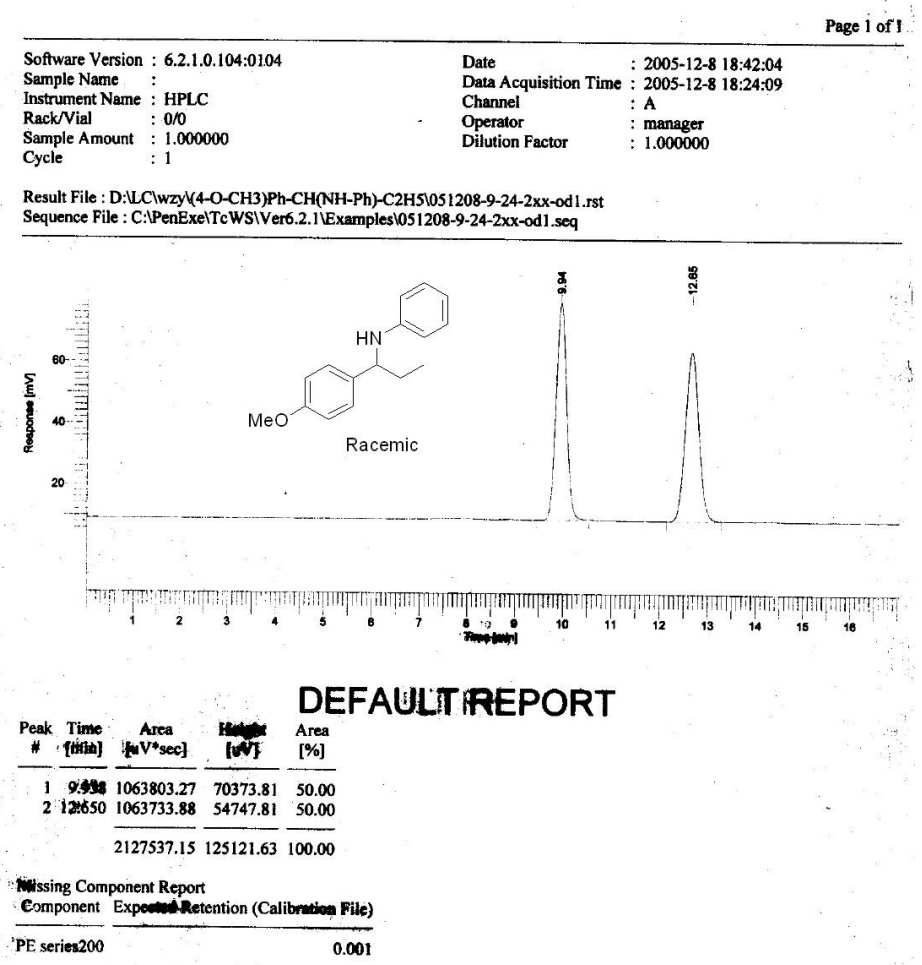

Page 1 of

$\begin{array}{llll}\text { Software Version } & : 6.2 .1 .0 .104: 0104 & \text { Date } & : \text { 2005-12-22 } \\ \text { Sample Name } & : \text { wzy9-37-1 } & \text { Data Acquisition Time } & : 2005-12-2 \\ \text { Instrument Name } & : \text { HPLC } & \text { Channel } & : \text { A } \\ \text { Rack/Vial } & : 0 / 0 & \text { Operator } & : \text { manager } \\ \text { Sample Amount } & : 1.000000 & \text { Dilution Factor } & : 1.000000 \\ \text { Cycle } & : 1 & & \end{array}$

Cycle

Result File : D:LClwzy)(4-O-CH3)Ph-CH(NH-Ph)-C2H51051222-9-37-1

Sequence File : C:PenExelTcWSIVer6.2.1 Examplesi051222-9-37-1.seq

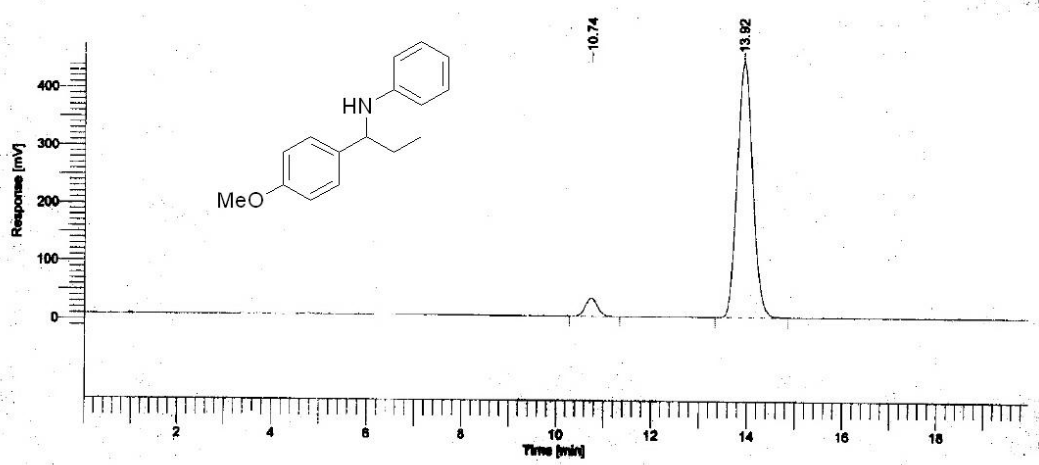

\section{DEFAULT REPORT}

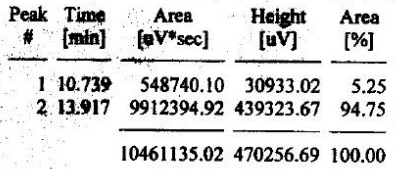

Missing Component Report

Cemponent Expectud Netention (Calibration File)

PE series200 
The HPLC spectra of $\mathbf{7 0}$

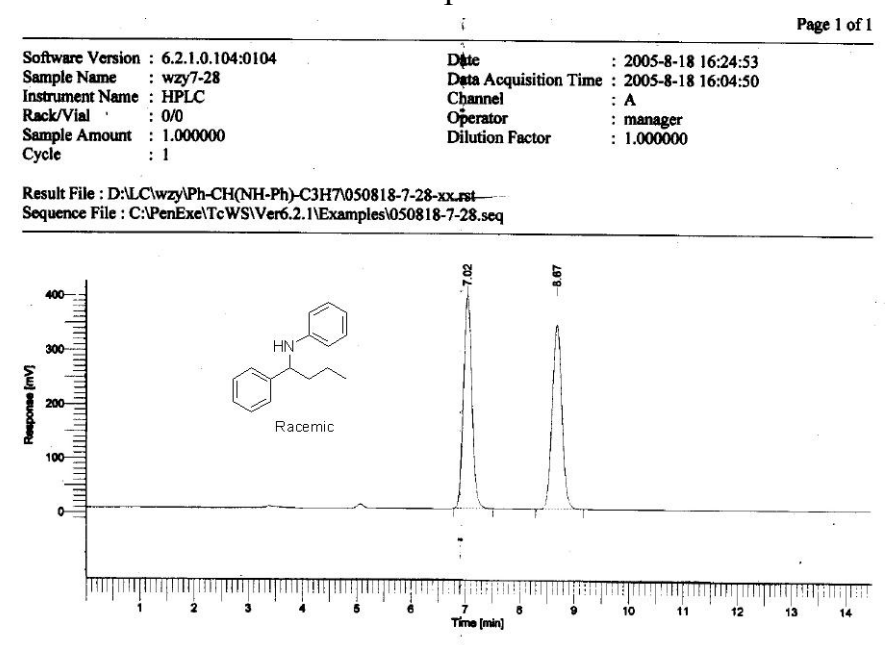

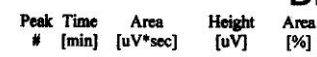

DEFAULT REPORT

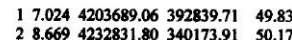

$\overline{8436520.86} \overline{733013.62} \frac{50.17}{100.00}$

Missing Component Report

Component Expected Retention (Calibration File)

PE series 200

Page 1,

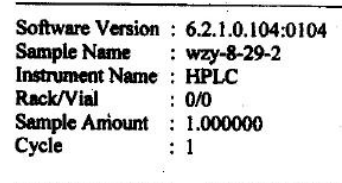

Result File : D:LClwzylPh-CH(NH-Ph)-C3H7050929-8-29-2.rst

Sequence File : C:IPenExelTcWSIVer6.2.1 Examples1050929-8-29-2.seq

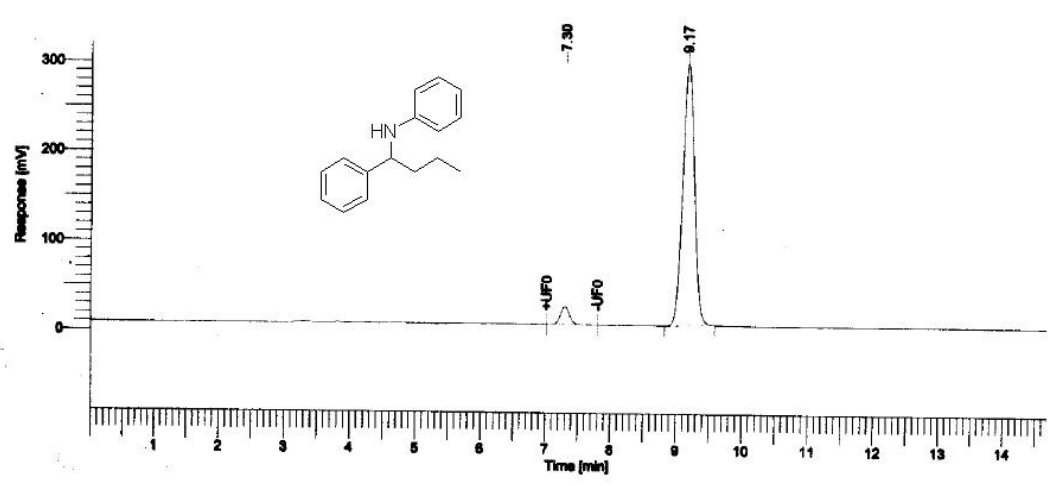

DEFAULT REPORT

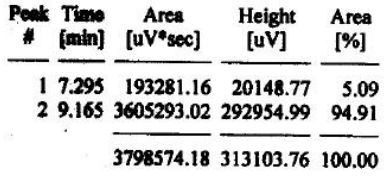

Misalng Component Report

Cotaponent Expected Retention (Calibration File)

PE series200 0.001 
Software Version : 6.2.1.0.104:0104

Sample Name : wzy8-31-2

$\begin{array}{ll}\text { Instrument Name : HPLC } \\ \text { Rack/Vial } & : 0 / 0\end{array}$

Sample Amount : 1.000000

Cycle
Date

Data Acquisition Time : $2006-4-1214: 34: 41$

Channel : A

Operator

Dilution Factor

Result File : D:LClwzylPh-CH(NH-Ph)-C3H71050929-8-31-2

Sequence File : C:PenExelTcWSIVer6.2.1 Examples1050929-8-31-2.seq

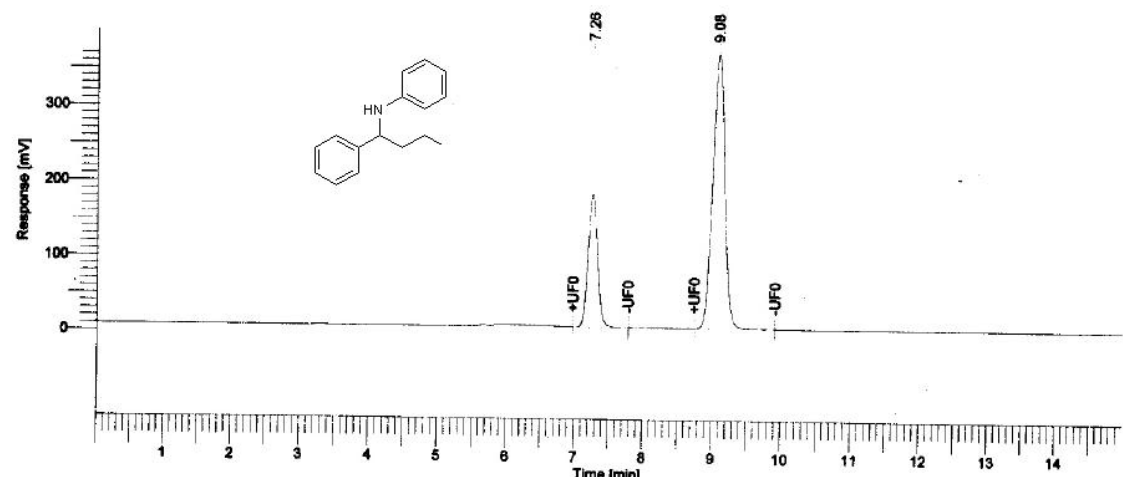

\section{DEFAULT REPORT}

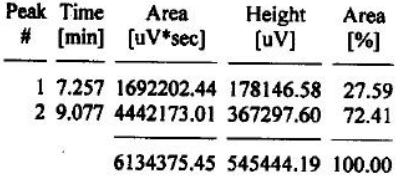

Missing Component Report

Component Expected Retention (Calibration File)

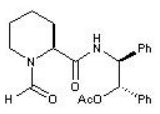

cat

$\begin{array}{ll}\text { PE series200 } & 0.001\end{array}$ 
The HPLC spectra of $\mathbf{7 p}$

\begin{tabular}{|c|c|c|c|c|}
\hline \multicolumn{2}{|c|}{ 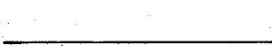 } & & & Pavelof \\
\hline $\begin{array}{l}\text { Software Version } \\
\text { Semple Name } \\
\text { Rustrument Name } \\
\text { Rect/Vial } \\
\text { Sumplo Amount } \\
\text { Cycle }\end{array}$ & $\begin{array}{l}6.2 \cdot 1.0 .104: 0104 \\
\text { HPLC } \\
0,0 \\
1.000000 \\
1\end{array}$ & $\begin{array}{l}\text { Date } \\
\text { Deat Aequistion Time } \\
\text { Channel } \\
\text { Operator } \\
\text { Dilution Factor }\end{array}$ & $\begin{array}{l}: 2006-1-515: 22: 32 \\
: 2006-1-5 \text { 14:40:43 } \\
: \text { A } \\
: \text { manager } \\
: 1.000000\end{array}$ & - \\
\hline $\begin{array}{l}\text { Renutt File: D:LC } \\
\text { Sequenoe File: : }\end{array}$ & Pe-CHAN & $\begin{array}{l}\text {-1xk-od 1.rst } \\
\mid 0-1 x x-0 d 1.209\end{array}$ & & \\
\hline
\end{tabular}
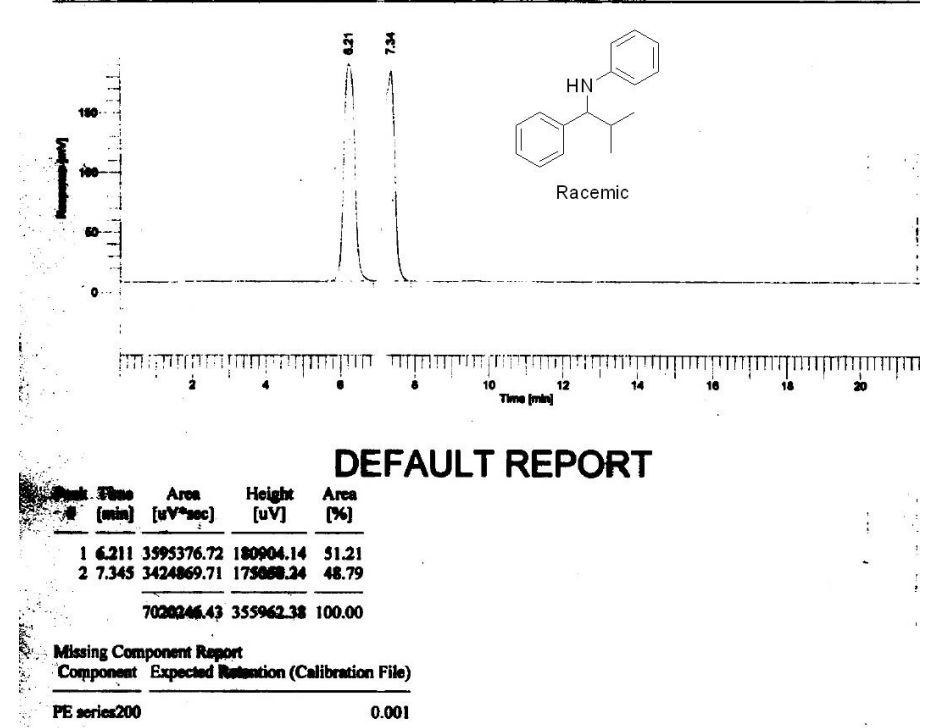

\begin{tabular}{lllll} 
& & & Page II \\
\hline Software Version $:$ & $6.2 .1 .0 .104: 0104$ & Date & $: 2006-1-620: 06: 53$ \\
Sample Name & $:$ WZY10-11-5 & Data Acquisition Time & $:$ 2006-1-6 19:52:47 \\
Instrument Name & $:$ HPLC & Channel & $:$ A \\
Rack/Vial & $: 0 / 0$ & Operator & $:$ manager \\
Sample Amount & $: 1.000000$ & Dilution Factor & $: 1.000000$ \\
Cycle & $: 1$ & &
\end{tabular}

Result File : D:LCCwzylPh-CH(NH-Ph)-CH(CH3)21060106-10-11-5 ist Sequence File : C:PenExelTcWSIVer6.2.11Examples 1060106-10-11-5.seq

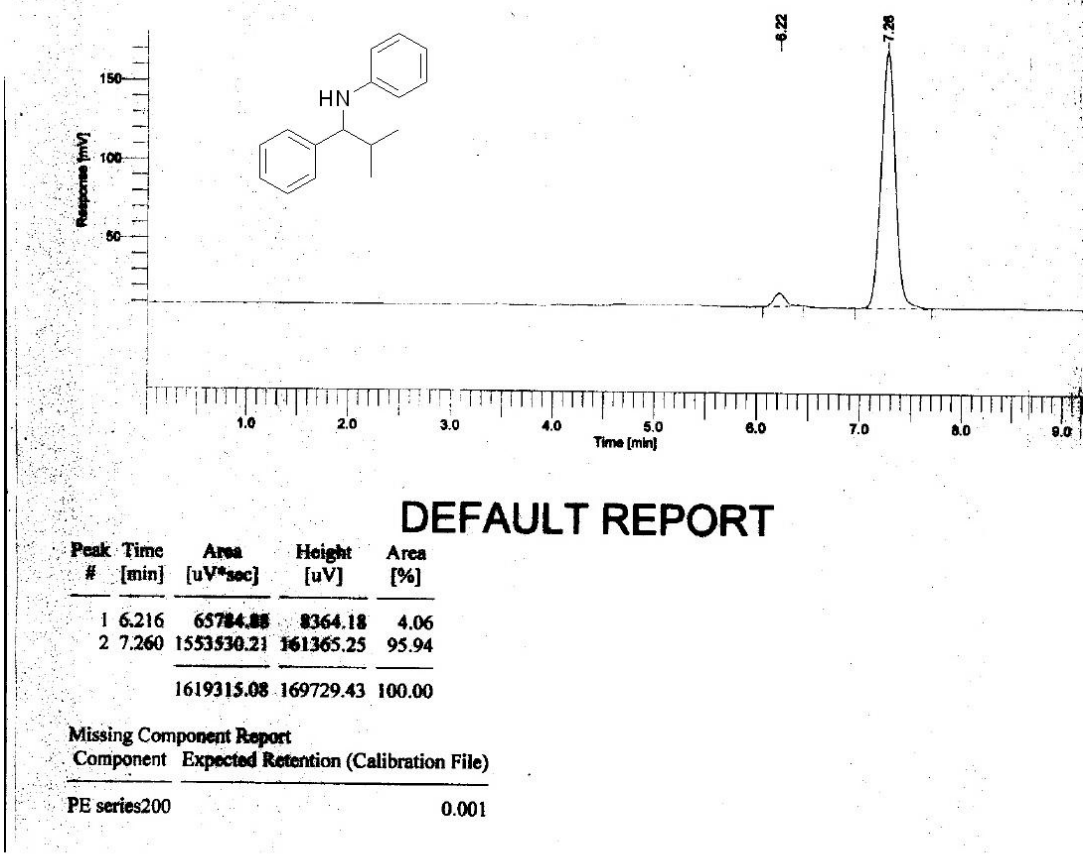




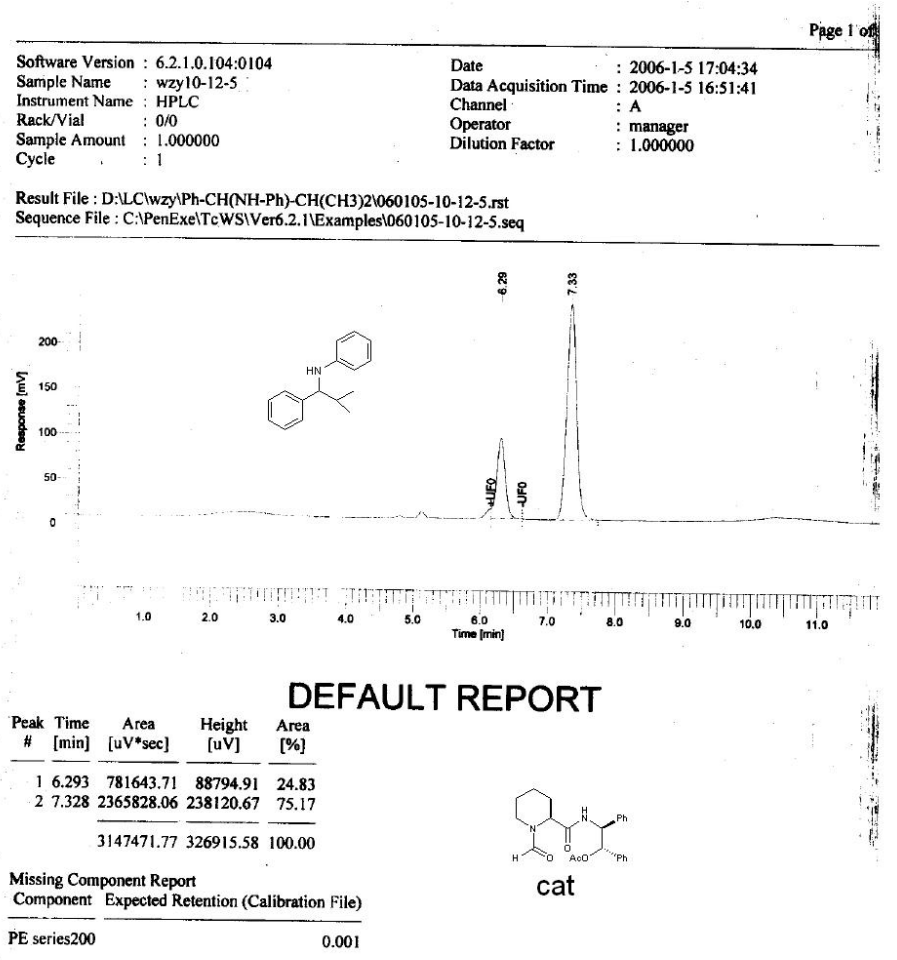


The HPLC spectra of $\mathbf{7 q}$

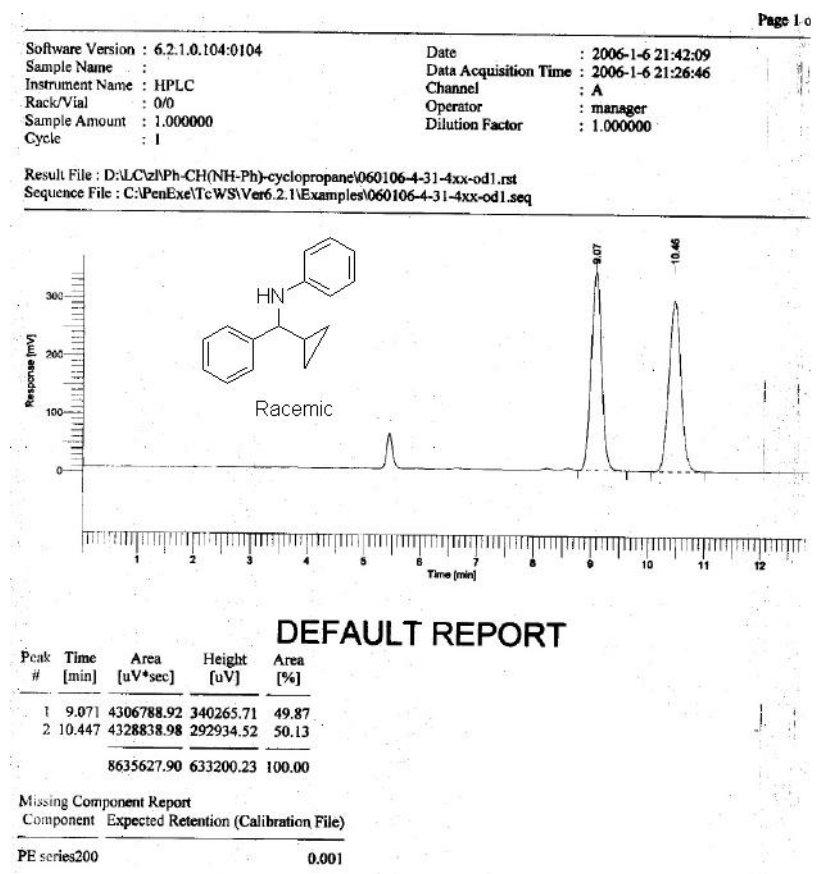

Page 10

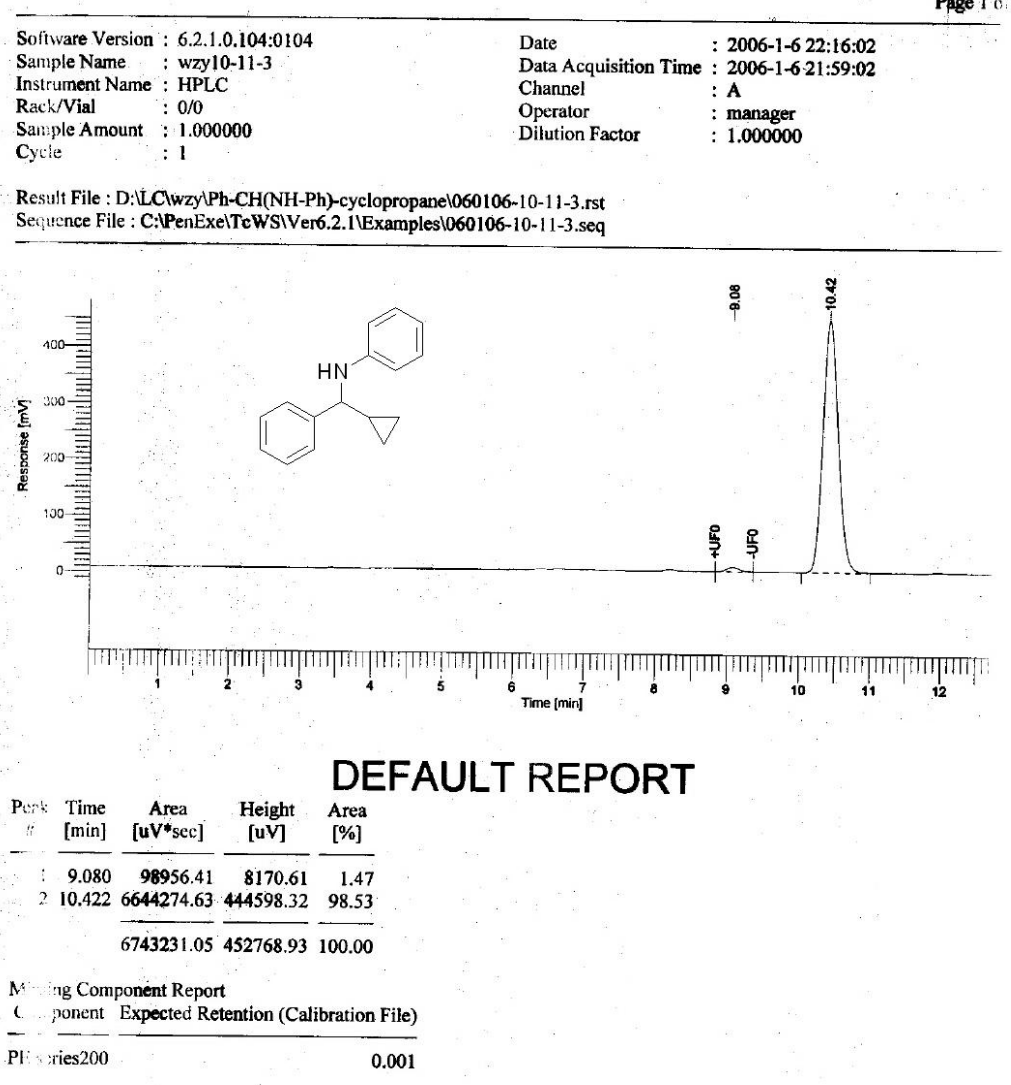


Software Version : 6.2.1.0.104:0104

Sample Name : wzy10-12-3

Rack/Vial $: 0 / 0$
Instrument Name

$\begin{array}{ll}\text { Sample Amount } & 1.000000 \\ \text { Cycle } & : 1\end{array}$

$\begin{array}{ll}\text { Date } & \text { 2006-1-6 21:58:19 } \\ \text { Data Acquisition Time } & : 2006-1-621: 42: 50\end{array}$

Data Acquisition Time : 2006-1-6 21:42:50

Operato

A

Result File : D:LCClwzylPh-CH(NH-Ph)-cyclopropane1060106-10-12-3

Seqnence File : C:PenExelTcWSiVer6 2.IExamplesi060106-10-12-3 st

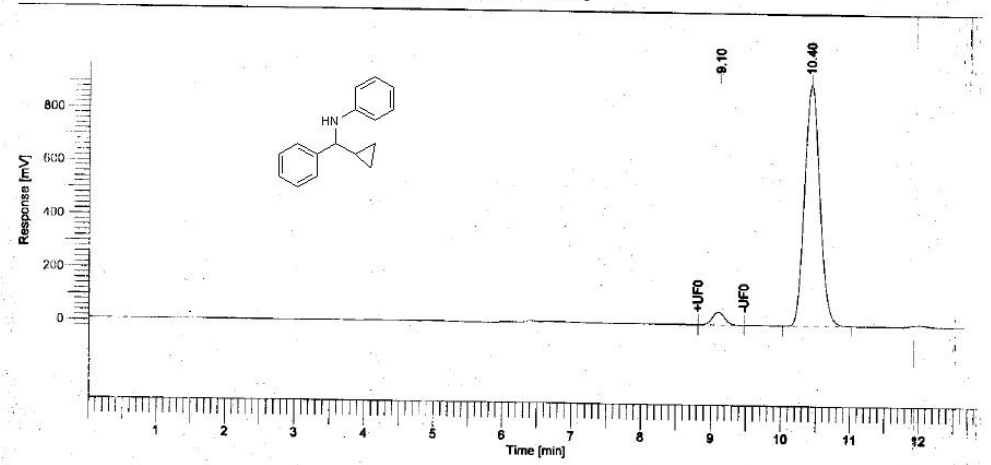

\section{DEFAULT REPORT}

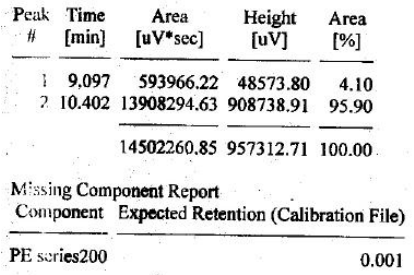

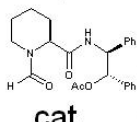

cat 
The HPLC spectra of $7 \mathbf{r}$

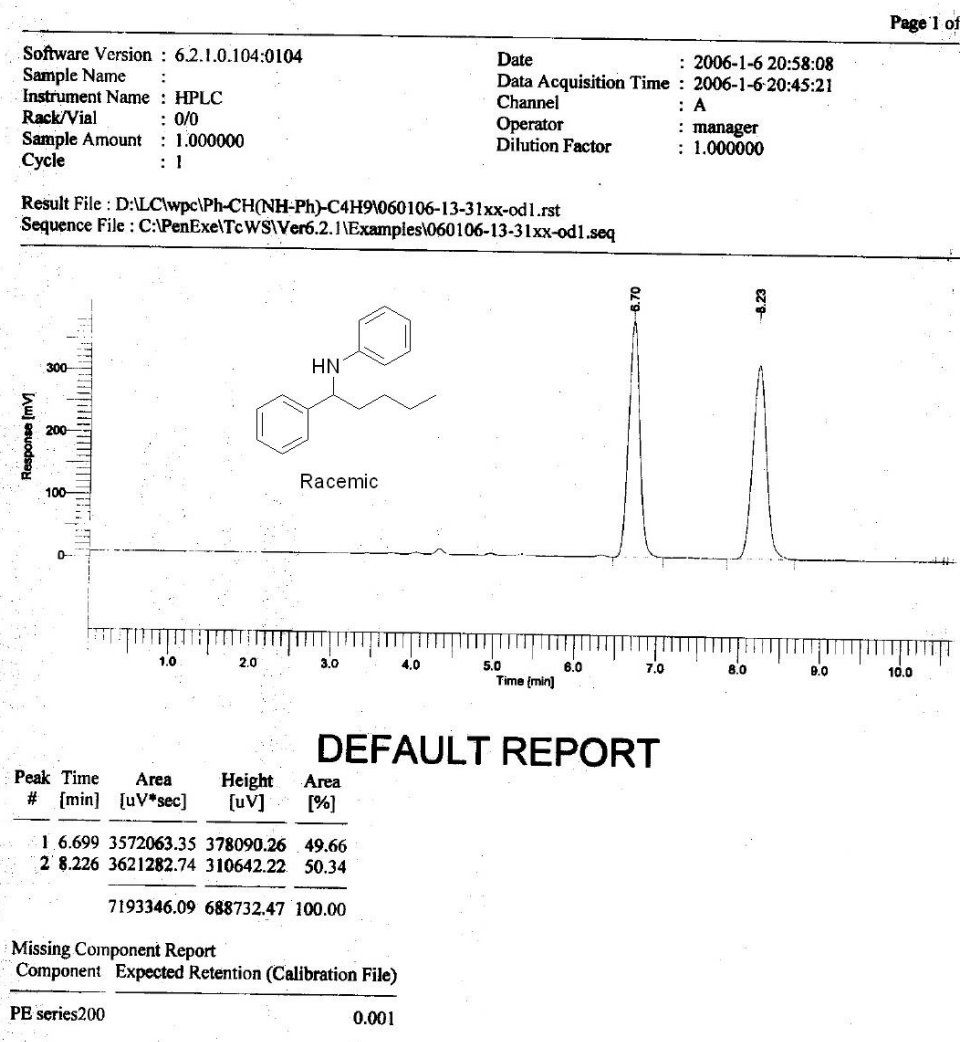

\begin{tabular}{|c|c|c|c|c|}
\hline $\begin{array}{l}\text { Software Version : } \\
\text { Sample Name } \\
\text { Instrument Name } \\
\text { Rack/Vial } \\
\text { Sample Amount } \\
\text { Cycle }\end{array}$ & $\begin{array}{l}: 6.2 .1 .0 .104: 0104 \\
: \text { wzyyl0-111-1 } \\
: \text { HPLC } \\
: 0 / 0 \\
: 1.000000 \\
: 1\end{array}$ & $\begin{array}{l}\text { Date } \\
\text { Data Acquisition Time } \\
\text { Channel } \\
\text { Operator } \\
\text { Dilution Factor }\end{array}$ & $\begin{array}{l}: \text { 2006-1-6 21:25:45 } \\
: 2006-1-621: 12: 06 \\
: \text { A } \\
: \text { manager } \\
: 1.000000\end{array}$ & \\
\hline
\end{tabular}

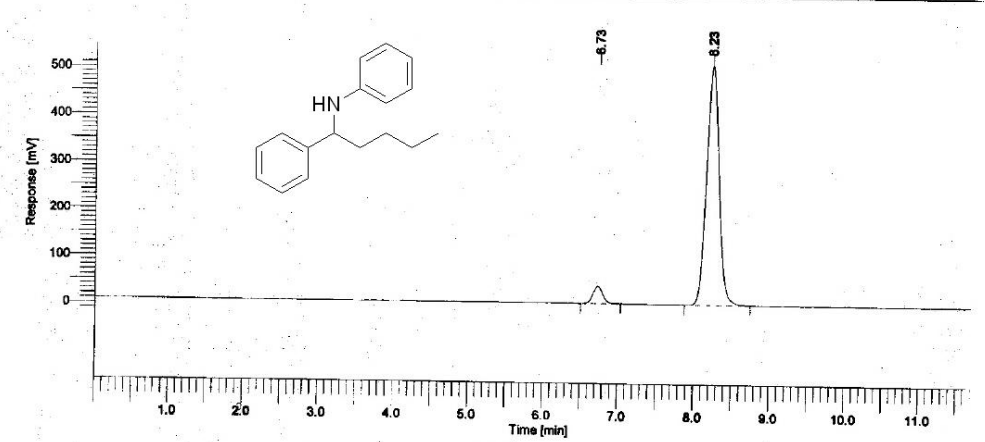

\section{DEFAULT REPORT}

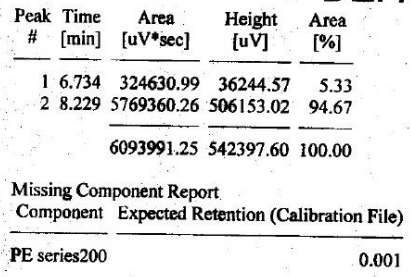




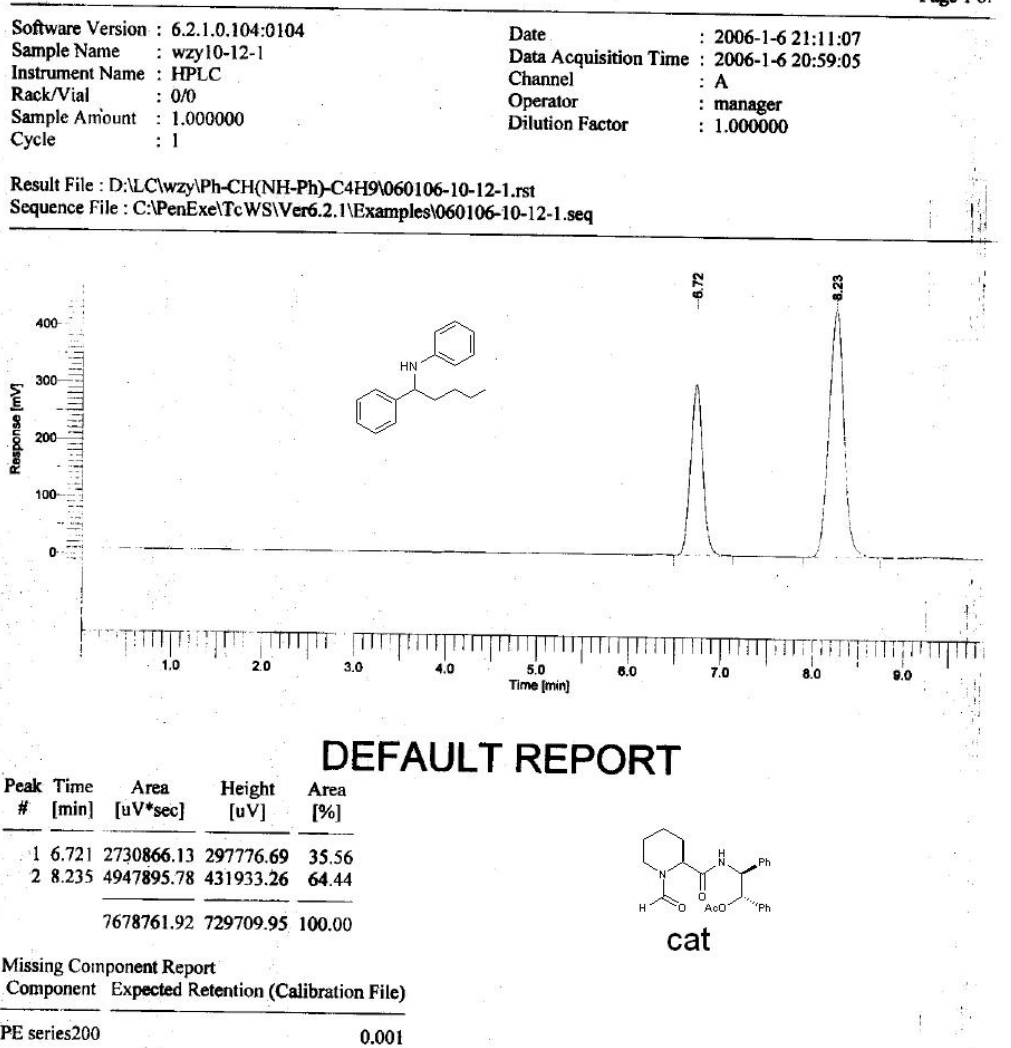


The HPLC spectra of $7 \mathbf{s}$
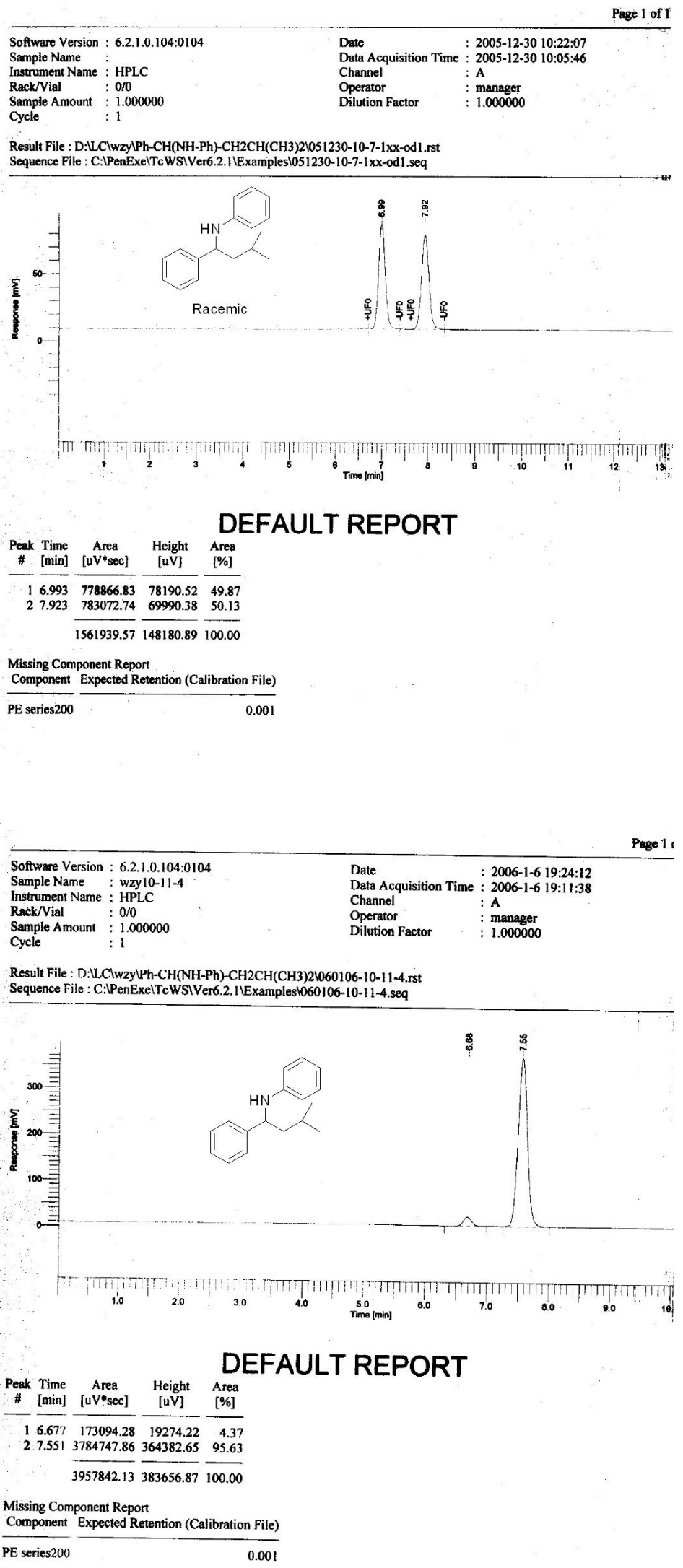
Software Version : 6.2.1.0.104:0104

Sample Name : : wzy10-12-4

Instrument Name : HPLC

Rack/Via

Date

Sample Amount : 1.000000

Channel

: 2006-1-5 14:25

Cycle

1

Operator $\quad:$ manager

Result File : D:LLClwzylPh-CH(NH-Ph)-CH2CH(CH3)21060105-10-12-4.5t Sequence File : C:IPenExelTcWSIVer6.2.1 Examplesi060105-10-12-4.seq

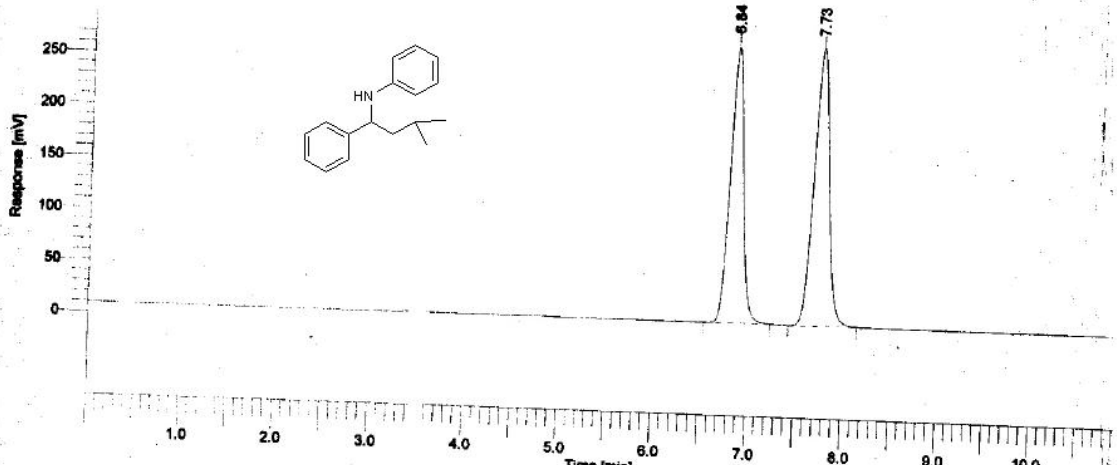

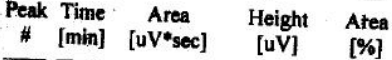

$$
\begin{aligned}
& 5458940.89531451 .10
\end{aligned}
$$

\section{DEFAULT REPORT}

Missing Component Report

Component Expected Retention (Calibration File)

PE series200

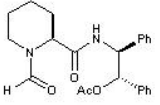

cat 